

\section{HARVARD UNIVERSITY.}

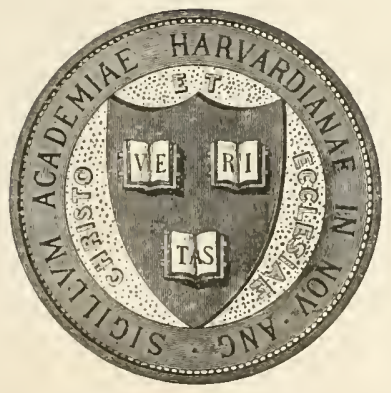

\section{LIBRARY}

OF THE

MUSEUM OF COMPARATIVE ZOÖLOGY 62,714 GIFT OF

$$
\text { Ithomad Pavoner }
$$

$$
\text { Decenter } 8.1923 .
$$




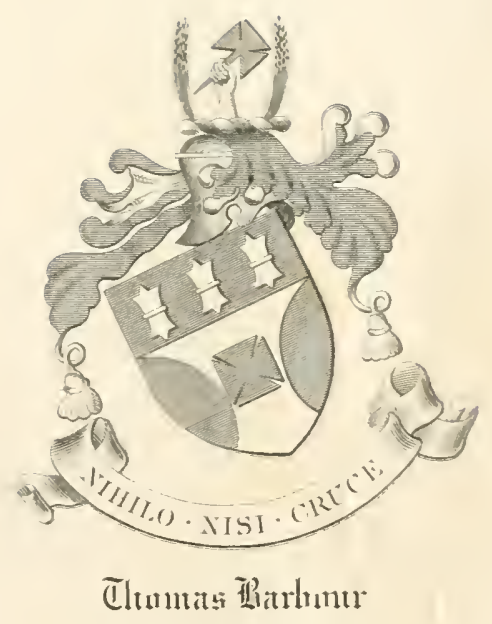




$$
\text { MCZ } 794 \text { Stejneger }
$$

Widener Photo Dept. order no. 79-5194

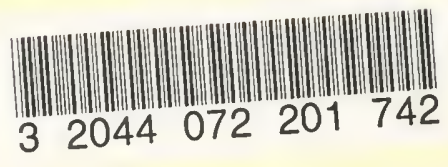


DEC $\quad 8 \quad 1923$ 



\title{
A CHECK LIST OF NORTH AMERICAN AMPHIBIANS AND REPTILES
}

BY

\author{
LEONHARD STEJNEGER \\ AND \\ THOMAS BARBOUR
}

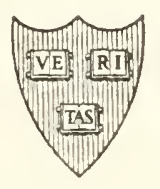

CAMBRIDGE

HARVARD UNIVERSITY PRESS 
$j$

COPYRIGHT, 1917
UARVARD UNIVERSITY PAE 


\section{INTRODUCTION}

AHECK LIS'T of North American Amphibians and A Reptiles has long been wished for by all students of American Herpetology. This list has been prepared generally upon the lines of the American Ornithologists' Union Check List of Birds, and following that example, it has included the species and subspecies which the authors deem valid ${ }^{1}$ and of certain occurrence in North America, north of the Rio Grande, and in Lower California, Mexico. Miller's List of North American Land Mammals (Bull. U. S. Nat. Mus., No. 79, 1912) has also been a valued guide.

This volume is the result of lists begun some years ago independently by both authors. The arrangement is on the following system: The higher groups and genera are in systematic sequence, the species are in alphabetic order. No attempt is made to give synonyms. Species not listed are believed to be invalid. 'The citation of original appearance of names is given for all except such (e. g., family names) as are formed automatically. Following the citation of the original description of each species comes the place of first appearance of the name in its present combinationaccurate in so far as we have been able to determine. References are added to Cope's North American Batrachia and to his Crocodilians, Lizards, and Snakes of North America. Both are frequently erratic and inaccurate, but large and excellent portions of both works are to a great extent from the pen of S. F. Baird, as painstaking an investigator as this country has produced. These monographs are in

1 By this statement it is not meant that the authors in all cases have verified the status of a number of more recently cstablished forms by critical study. 'The judgment of reliable workers has been accepted where no special reason appeared to contraindicate the validity of the form. As for the admission of subspecies - or rather trinoninal designation - for certain forns no spccial attempt has been made at consistency, the authors on principle leaning towards binominals in all cases where the need of trinominals has not bcen clearly established. 
widespread use, and constitute the most recent attempts toward complete lists. In the case of the difficult genus Thamnophis references are given to Ruthven's excellent review. Common names for reptiles and amphibians are in great confusion, and are often of generic rather than of specific application. No attempt is made to recognize any except those which are in well-established usage. The host which have been recently coined by various writers are frequently inapt and almost surely unlikely to survive. It is entirely possible that some vernacular names really often used have escaped our notice, since many are in restricted local use. The type localities are as nearly exact as it is possible to determine. This cannot be said regarding the ranges. Many are obviously faulty, but a sincere attempt has been made to collect records of authentic captures; however, with a literature so extensive and so scattered, records have almost surely been overlooked. In many cases, our knowledge does not warrant drawing hard and fast lines delimiting a form's occurrence, and we often state ranges in purposely general terms. There still is opportunity for valuable field work to determine the precise distribution of many of our most common species. An example is the recent discovery that Virginia valeriae is far from rare in parts of New Jersey.

Our hearty thanks are due to many for information and advice, especially to Samuel Henshaw, John Van Denburgh, and Alex. G. Ruthven.

G. K. Noble has assisted considerably in verifying references and searching literature, and for this assistance he has our grateful remembrance.

T. B. 
Intimation to Second Edition.

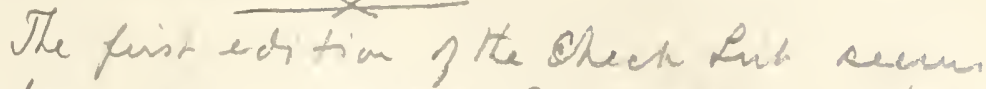

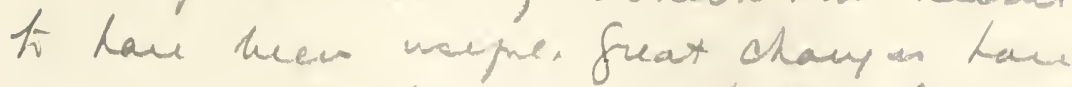
cone un ma hervestle. of the doth duncan

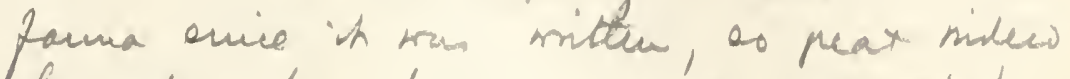
that then has been a general request for

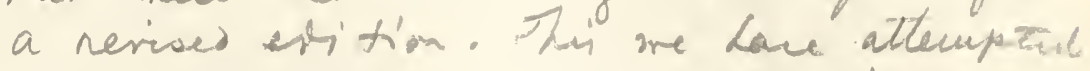
$m$ as rimpatiac mermen as prosibuce. Probable none will apes with all me conclusions. The reason for disusing this revision now is that the percent sear no the as for a thus an any late date, in view 8 the fact that the last mons will not he arid for yeans to coom.

Wage varies sorer man to the printing of accents on spanish words that no attempt has been mate to satisfy aid the puristic conventions in this connection. Thanks are due to nest of our hood American co-ronkers in Herpetology for advice and avivatura. 



\section{AMPHIBIA (CLASS)}

Linné, Syst. Nat., Ed. 10, Vol. 1, 1758, p. 194

\section{CAUDATA (ORDER)}

Oppes, Ordn. Rept. 1811, p. 79

\section{PROTEIDA (SUBORDER)}

Cope, Journ. Ac. Nat. Sci. Phila., Ser. \&, Vol. 6, 1866, p. 102

\section{NECTURIDAE $(F A M I L Y)$}

Necturus (GENUS)

Rafinesque, Journ. Phys. Chim. Hist. Nat., Vol. S8, June, 1819, p. 418 TYPE: maculatus $=$ maculosus

Necturus maculosus (RAFINESQUE)

MUDPUPPY, WATERDOG

Sirena maculosa Rafinesque

Amer. Month. Mag. Crit. Rev., Vol. 4, No. 1, Nov. 1818, p. 41.

Necturus maculosus Rafinesque

Ann. Nat., Lexington, No. 1, March 29,1820 , p. 4.

Necturus maculatus Cope

Bull. U.S. Nat. Mus., No. 34, 1889, p. 23, fig. 3.

TYPE LOCALITY: Found in the Ohio River.

RANGE: Tributaries of the Great Lakes, the Mississippi River system, the upper Hudson River, and Lake Champlain.

\section{$\checkmark$ Necturus punctatus (GiBBns)}

Menobranchus punctatus GibBes

Proc. Amer. Assoc. Adv. Sci., Charleston, 1850, p. 159.

Necturus punctatus Cope

Bull. U. S. Nat. Nus., No. 34, 1889, p. 27, fig. 4.

TYPE LOCALITY: Dr. Schoolbred's plantation on the South

Santee River, a few miles from its mouth, South Carolina.

RANGE: Fresh waters of North and South Carolina, especially rice field ditches. 


\title{
TYPHLOMOLGIDAE ( FAMILY)
}

\author{
Typhlomolge ( $G E N U S)$ \\ Stejneger, Proc. U.S. Nat., Mus., Tol. 18, 1896, p. 620 \\ TYPE: rathbuni
}

Typhlomolge rathbuni STEJNEger

Typhlomolge ralhbuni STEJNEGER

Proc. U. S. Nat. Mus., Vol. 18, Apr. 15, 1896, p. 620.

TYPE LOCAlity: Artesian well 188 feet deep, at U.S. Fish Com-

mission Station, San Marcos, Hays Co., Texas.

RANGE: Known from vicinity of the type locality only.

\section{MUTABILIA (SUBORDER)}

Merrem, Syst. Amph., 1820, p. 166

\section{AMPHIUMIDAE (FAMILY)}

Amphiuma ( $G E N C S)$

Garden, Smith`s Correspondence of Limnacus, Vol. 1, 1821, p. 599

TYPE: means

Amphiuma means Garden

BLIND EHL, CONGO HEL, CONGO SNAKE

Amphiuma means Garden

Smitl's Correspondence of Linnaeus, Vol.1, 1821, p.599, (desc. p.333). Amphiuma means Cope

Bull. U. S. Nat. Mus., No. 34, 1889, p. 216.

TYPE LOCALITY: Not stated, but from the context evidently either Charleston or East Florida.

RANGE: From Virginia south to Florida, west to Louisiana.

Amphiuma tridactylum Curier

Amphiuma tridactylum Cunier

Mém. Mus. Hist. Nat. Paris, Vol. 14, 1897, p. 7, pl. 1, figs. 4-6, pl. 2, figs. 9-18.

TYPE LOCAlity: New Orleans, Louisiana.

RA NGE: Northern Florida to Southern Mississippi and Louisiana, up the Mississippi Valley at least to Missouri. 




\section{CRYPTOBRANCHIDAE (FAMILY) \\ Cryptobranchus ( $G E N U S$ ) \\ LeuCKarT, Isis, 1821, Lit. Anz., p. 259 \\ TYPE: gigantea $=$ alleganiensis}

\section{Cryptobranchus alleganiensis (DAUDiN)}

\section{HELL-BENDER}

Salamandra alleganiensis DAUDIN

Hist. Nat. Rept., Vol. 8, 1803, p. 231.

Cryptobranchus alleghaniensis VAN DER HOEVEN

Tijdschr. Nat. Geschied. Physiol., Vol. 4, 1837, p. 384.

('ryptobranchus allegheniensis COPE

Bull. U. S. Nat. Mus., No. 34, 1889, p. 38, fig. .5.

TYPE LOCALITY: Alleghany Mts. in Virginia.

RANGE: Western New York, the Great Lakes system, Iowa and southward to Georgia and Louisiana.

\section{SALAMANDRIDAE $(F A M I L Y)$}

Notophthalmus ( GENUS)

Rafixesque, Ann. Nat.. Lexington, No. 1, March 92, 1890, p. 5 TYPE: miniatus $=$ viridescens

\section{Notophthalmus meridionalis (COPE)}

Diemyctylus miniatus, subsp. meridionalis COPE

Bull. U. S. Nat. Mus., No. 17. 1880, p. 30.

Diemyctylus viridescens meridionalis $\mathrm{COPE}$

Bull. U. S. Nat. Mus., No. 34, 1889, p. 211, fig. 54.

TYPE LOCALITY: Matamoros, Tamaulipas, Mexico.

RANGE: Southern Georgia to Louisiana, Texas and Tamaulipas.

Notophthalmus torosus (Eschscholtz)

GIANT NEWT, CALIFORNIA NEW'T

Triton torosus EschschoLT'

Zool. Atlas, Pt. 5, 1833, p. 12, pl. 21, fig. 15.

Notophthalmus torosus BAIrD

Journ. Ac. Nat. Sci. Phila., Ser. ․, Vol. 1, 1849, p. 284.

Diemyctylus torosus COPE

Bull. U. S. Nat. Mus., No. 34, 1889, p. 204 , fig. 51.

TYPE LOCALITY: Surroundings of the Bay of San Francisco, California.

RA NGE: California, Oregon and Washington. 
Notophthalmus viridescens viridescens (RAFINESQUE)

COMMON NEWT (AQUATIC FORM) RED EFT OR EBBET (TERRESTRIAL FORM)

Triturus viridescens RAFinesque

Ann. Nat., Lexington, No. 1, March 29,1820 , p. 5.

Notophthalmus viridescens BATRD

Journ. Ac. Nat. Sci. Phila., Ser. , Vol. 1, 1849, p. 284.

Diemyctylus viridescens $\mathrm{COPE}$

Bull. U. S. Nat. Mus., No. 34, 1889, p. 207, figs. 52-53.

TYPE LOCAlity: Lake George, Lake Champlain.

RANGE: Eastern North America, Hudson Bay to Texas, west

Illinois, Michigan, Missouri to Oklahoma.

Notophthalmus viridescens vittatus (H. Garman)

Diemyctylus viridescens var. vittatus $\mathbf{H}$. Garman

Journ. Cincinnati Soc. Nat. Hist., Vol. 19, 1897, p. 49.

TyPE Locality: Wilmington, North Carolina.

RANGE: Only known from type locality.

\section{AMBYSTOMIDAE (FAMILY)}

Ambystoma ( $G E N U S)$

Tschudr, Mém. Soc. Sci. Nat. Neuchâtel, 1838, p. 99 TYPE: subviolacea $=$ maculatum

Ambystoma annulatum CoPE

Amblystoma annulatum COPE

Proc. Amer. Philos. Soc., Vol. 23, 1886, p. 525.

Linguaelapsus annulatus COPE

Bull. U. S. Nat. Mus., No. 34, 1889, p. 115. fig. 95.

TYPE LOCALITY: Unknown.

RANGE: Arkansas.

Ambystoma aterrimum CoPE

Amblystoma aterimum CopE

Proc. Ac. Nat. Sci. I'hila., 1867, p. 201.

Chondrotus aterrimus COPE

Bull. U. S. Nat. Mus., No. 34, 1889, p. 109, fig. 23.

TYPE LOCALITY: Northern Rocky Mountains (in Montana).

RANGE: Northern Rocky Mountains.

Ambystoma cingulatum Cope

Amblystoma cingulatum CopE

Proc. Ac. Nat. Sci. Phila., 1867, p. 205.

Chondrotus cingulatus COPE

Bull. U. S. Nat. Mus., No. 34, 1889, p. 100, p. 455. fig. 116. 


TYPE LOCALITY: Grahamville, South Carolina.

RANGE: South Carolina to Northern Florida.

\section{Ambystoma conspersum Cope}

Ambystoma conspersum Cope

Proc. Ac. Nat. Sci. Phila., 1859, p. 123.

Amblystoma conspersum CoPE

Bull. U. S. Nat. Mus., No. 34, 1889, p. 61, fig. 10.

TYPE LOCALITY: Londongrove, Chester Co., Pennsylvania.

RANGE: Pennsylvania to Georgia.

\section{Ambystoma decorticatum Cope}

Amblystoma decorticatum Cope

Proc. Amer. Philos. Soc., Vol. 23, 1886, p. 592.

Chondrotus decorticatus COPE

Bull. U. S. Nat. Mus., No. 34, 18s9, p. 107, fig. 然.

TYPE LoCAlity : Port Simpson, British Columbia.

RANGE: Coastal region of South Eastern Alaska and British Columbia.

\section{Ambystoma epixanthum Cope}

Amblystoma epixanthum Cope

Proc. Ac. Nat. Sci. Phila., 1883, p. 16.

Amblystoma epixanthum $\mathrm{CoPE}$

Bull. U. S. Nat. Mus., No. 34, 1889, p. 9\%. fig. 18.

TYPE LOCALITY: "Swamp near head of South Boise River, on the south side of the Sawtooth Mts., Idaho."

RANGE: Idaho and Montana.

\section{Ambystoma jeffersonianum (GREEN)}

\section{Salamandra jeffersoniana GreeN}

Contr. Maclur. Lyc., Vol. 1, No. 1, Jan. 1827, p. t, pl. 1, fig. 1.

Ambystoma jeffersoniana BAIRD

Journ. Ac. Nat. Sci. Phila., Ser. 2, Vol. 1, 1849, p. 283.

Amblystoma jeffersonianum CoPE

Bull. U. S. Nat. Mus., No. 34, 1889, p. 89, figs. 15-16.

TYPE LOCALITY : Near Jefferson College, Cannonsburg, Pennsylvania.

RANGE: Canada (to Hudson Bay) and New England, southward to Virginia, Illinois and Arkansas.

\section{Ambystoma lepturum Cope}

Amblystoma lepturum CoPE

Proc. Amer. Philos. Soc., Vol. 23, 1886, p. 524. 


\section{Linguaelapsus lepturus CoPE}

Bull. U. S. Nat. Mus., No. 34, 1889, p. 116, fig. 26.

TYPE LOCALITY: Unknown.

RANGE: Unknown.

\section{Ambystoma macrodactyium BAIRD}

Ambystoma macrodactyla BAIRD

Journ. Ac. Nat. Sci. Phila., Ser. 2, Vol. 1, 1849, p. 292.

Amblystoma macrodactylum $\mathrm{Cope}$

Bull. U. S. Nat. Mus., No. 34, 1889, p. 95, fig. 17.

TYPE LOCALITY: Astoria, Oregon.

RANGE: Northern California to British Columbia, Montana.

\section{Ambystoma maculatum (SHAw)}

SPOTTED SALAMANDER

Lacerta maculata SHAw

Gen. Zool. Vol. 3, Pt. 1, 1802, p. 304. ${ }^{1}$

Ambysloma maculatum Stejneger

Proc. Biol. Soc. Washington, Vol. 15, Dec. 16, 1909, p. 239.

Amblystoma punctatum Cope

Bull. U. S. Nat. Mus., No. 34, 1889, p. 56, fig. 9.

ty pe Locality : Carolina (based on Catesby, Nat. Hist. Carolina, Ed. 1, Appendix, 1748, pl. 10, fig. 10.)

RANGE: Nova Scotia west to Wisconsin, southward to Georgia and Texas.

\section{Ambystoma microstomum CopE}

Amblystoma microstomum Cope

Proc. Ac. Nat. Sci. Phila., 1861, p. 123.

Chondrotus microstomus Cope

Bull. U. S. Nat. Mus., No. 34, 1889, p. 101, fig. 19.

TYPE LOCALITY: Wabash, Ohio. ${ }^{2}$

RANGE: South Carolina to Texas, northward to Ohio, Illinois and possibly Canada.

\section{Ambystoma opacum (Gravenhorst)}

\section{MARBLED SALAMANDER}

Salamandra opaca Gravenhorst.

Vergl. Uebers. Zool. Syst., 1807, p. 431.

Ambystoma opaca BAIRD

Journ. Ac. Nat. Sci. Phila., Ser. 2, Vol. 1, 1849, p. 283.

1 This is Lacerta punctata of Linné, Syst. Nat., Ed. 12, 1766, p. 370, but not L. punctata of Linné, Syst. Nat., Ed. 10, 1758, p. 209, or Ed. 12, 1766, p. 369.

2 Possibly incorrect, cf. Fowler and Dunn, Proc. Ac. Nat. Sci. Phila., Vol. 69, 1917, p. 11. It is probably Wabash River, Indiana. 




\section{Amblystoma opacum CoPE}

Bull. U. S. Nat. Mus., No. 34,1889 , p. 54 , fig. 8.

TYPE LOCALITY: New York.

RANGE: From Massachusetts to Florida, west to Louisiana and Texas, Mississippi basin north to Arkansas, Missouri, Indiana and Illinois.

\section{Ambystoma paroticum BAIRD}

Amblystoma paroticum BAIRD,

in Cope Proc. Ac. Nat. Sci. Phila., 186\%, p. 200.

Chondrotus paroticus COPE

Bull. U. S. Nat. Mus.. N. 34, 1889, p. 105, fig. 21.

TYPE LOCALITY: Chiloweyuck Lake, British Columbia

RANGE: Northern California to British Columbia, Vancouver Island.

\section{Ambystoma stejnegeri RтTнгі}

Ambystoma stejnegeri Ruthvex

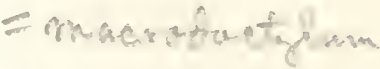

Proc. U. S. Nat. Mus., Tol. 41, Jan. 29, 1912, p. 517.

TYPE LOCALITY: Bloomfield, Davis Co., Iowa.

RANGE: Southeastern Iowa.

\section{Ambystoma talpoideum (HoLBRooK)}

MOLE SALAMANDER

Salamandra talpoidea HoLBrook

N. Amer. Herp.. Ed. 1, Vol. 3, 1838, p. 117, pl. 99.

Ambystoma talpoideum GRAY

Cat. Batr. Grad. Brit. Mus., 1850, p. 36.

Amblystoma talpoideum Cope

Bull. U. S. Nat. Mus.. No. 34. 1859, p. 59 , fig. 7.

TYPE LOCALITY: Sea Islands on the border of South Carolina. RANGE: South Atlantic and Gulf States, Louisiana to Illinois.

\section{Ambystoma tenebrosum ${ }^{1}$ BAIRD and Girard}

Amblystoma tenebrosum BAIRD and Girard

Proc. Ac. Nat. Sci. Phila., Vol. 6, 1852, p. 174.

Chondrotus tenebrosus CopE

Bull. U. S. Nat. Mus., No. 34, 1889, p. 111, fig. 24.

TYPE LOCALITY: Oregon.

RANGE: Central California to Southern coastal region of British

Columbia.

1 According to Yan Denburgb Dicamptodon ensalus (Eschscholtz Zool. Atlas, pt. 5, 1833, p. 6, pl. 2२), is a synonym of Ambystoma tenebrosum Baird and Girard; until, however, it is possible to examine the type, if it still exists at Dorpat or Rostock, it seems better to consider this allocation only provisional, in view of the fact that the figure in the Zoologisches Atlas is not very similar to the Ambystoma. We do not, however, include it in the list.

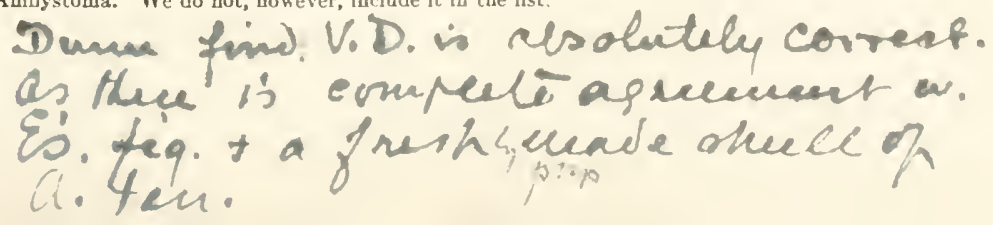




\section{Ambystoma texanum (Matthes)}

Salamandra texana MATthes

Allg. deutsche naturh. Zeitschr., N. S., Vol. 1, 1855, p. 266.

Amblystoma texanum BaIrD

U. S. Nat. Mex. Bound. Surv., Vol. 2, 1859, Rept. p. 29, pl. 35, fig. 15. Chondrotus texanus COPE

Bull. U. S. Nat. Mus., No. 34, 1889, p. 104, fig. 20.

TYPE LOCALity: "Das erste Exemplar fand ich . . . in Urwalde am Rio Colorado, das zweite ebenfalls ... im Cumming's Creeck (sic) Bottom, Fayette County."

RANGE: Texas.

\section{Ambystoma tigrinum (Green)}

TIGER SALAMANDER

Salamandra tigrina GreeN

Journ. Ac. Nat. Sci. Phila., Vol. 5, 1825, p. 116.

Ambystoma tigrina BAIRD

Journ. Ac. Nat. Sci. Phila., Ser. 9. Vol. 1, 1849, p. 284.

Amblystoma tigrinum $\mathrm{COPE}^{1}$

Bull. U. S. Nat. Mus., No. 34, 1889, p. 68, figs. 12-13.

TYPE LOCALity : Near Moorestown, New Jersey.

RANGE: Widely distributed over almost all the United States, east of the Rocky Mts., and extending southward over the north ern portion of the Mexican plateau.

\section{Ambystoma trisruptum Cope}

Amblystoma trisruptum COPE

Proc. Ac. Nat. Sci. Phila., 1867 , p. 194.

Amblystoma trisruptum COPE

Bull. U. S. Nat. Mus., No. 34, 1889, p. 86.

type locality: Ocate Creek, New Mexico.

RANGE: New Mexico.

\section{Ambystoma xiphias Cope}

Amblystoma xiphias Cope

Proc. Ac. Nat. Sci. Phila., 1867, p. 192.

Amblystoma xiphias Cope

Bull. U.S. Nat. Mus., No. 34, 1889, p. 87, fig. 14.

TYPE LOCALITY: Columbus, Ohio.

RANGE: Ohio.

I According to a note in Cope's bandwriting in the jar containing the type of $A$. copeianum Hay, be considered it the same as A. tigrinum, although it is rcognized in his Batrachia of North America. 


$$
\text { • }
$$


Rhyacotriton (Ienur)

Dumn, Proc. Kew Enq. Zool. Chublin, 1920, p. 56 Type: olympicus.

Rhyacotrition, alympe an. Dum.

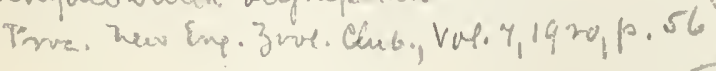

Batrachoseps leucopers Dumn.

Batroch oseps lencopus Dumm.

Copeia, ho 109 , aup 15, 1922,10.60

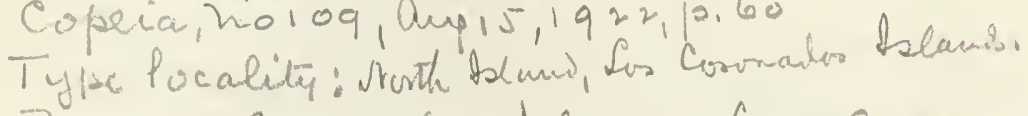

Range: Cononavos beaues, Lorrer Califormis.

Batrachoseps catalina Dum.

Batrachosess catalinac omu.

Coppeia, 10. 109 , anc. $15,1922,10.62$

Type fvealiti: Santio Cataleine Sland.

Promge: samae. 


\section{Ranodon ( $G E N U S)$}

Kessleir, Bull. Soc. Natural. Moscou, Vol. 39, pt. 1, 1866, p. 130 Rhycectidio TY PE: sibiricns

Ranodon olympicus (GAIGE)

Ranodon olympicus GaIGE

Occ. Papers Mus. Zoöl. Univ. Michigan, No. 40, May 30, 1917, p. \&, pl. 1.

TYPE LOCALITY: Lake Cushman, Washington.

RANGE: The Olympic Mts. Washington

\section{PLETHODONTIDAE $(F A M I L Y)$}

Batrachoseps ( $G E N U S$ )

Bonaparte, Iconogr. Fauna Italica, Vol. 2, fasc. 26, 1839.

fol. $131 * * *$ (not paged)

T $\mathrm{P} \mathrm{E}$ : altenuatus

\section{Batrachoseps attenuatus (EsCHSCHOLTZ)}

Salamandrina attenuata EschschoLTz

Zool. Atlas, Pt. 5, 1833, p. 1, pl. 21, figs. 1-14.

Batrachoseps altenuatus CoOper

Pacif. R. R. Surv., Vol. 12, pt. 3, 1858, Zoöl., p. viii, pl. 31, fig. 5.

Batrachoseps attenuatus COPE

Bull. U.S. Nat. Mus., No. 34, 1889, p. 197, fig. 28.

TYPE LOCALITY : Environs of Bay of San Francisco, Cal.

RA NGE: Southwestern Oregon, California, and Lower California.

Batrachoseps caudatus CopE

Batrachoseps caudatus COPE

Bull. U. S. Nat. Mus., No. 34, 1889, p. 126, fig. 27.

type Locality: Hassler Harbor, Alaska. (Probably on Anette

Island, S. E. Alaska.)

RANGE: Northern Channel Islands, Alaska.

Batrachoseps pacificus (COPE)

IIcmidactylium pacificum CoPE

Proc. Ac. Nat. Sci. Phila., 1865, p. 195.

Batrachoseps pacificus Cope

Proc. Ac. Nat. Sci. Phila., 1869, p. 99.

Batrachoseps pacificus COPE

Bull. U. S. Nat. Mus., No. 34, 1889, p. 129.

TyPE LOCAlity: Santa Barbara (?), Cal.

RANGE: Islands off the coast of California. 


\section{Batrachoseps major CAMP}

Batrachoseps major CAMP

Univ. California Publ. Zoöl., Vol. 12, No. 12, Apr. 2, 1915, p. 327.

Type Locality : Sierra Madre, 1,000 feet elevation, Los Angeles Co., California.

RANGE: Southwestern California.

\section{Hemidactylium ( $G E N U S$ )}

'T'schudi, Mém. Soc. Sci. Nat. Neuchâtel, 1838, p. 94

Hemidactylium scutatum (SCHLEGEL)

FOUR-TOED SALAMANDER

Salamandra scutata ScliLEgel

Fauna Japonica, Saur. Batr., 1838, p. 119.

Hemidactylium scutatum Tscuud

Mém. Soc. Sci. Nat. Neuchâtel, 1838, p. 94.

Hemidactylium scutatum COPE

Bull. U. S. Nat. Mus.. No. 34, 1889, p. 130, fig. 29.

TyPE LOCALITY: Nashville, Tennessee.

RANGE: Massachusetts, southern Canada westward to Michigan, south to Georgia and the Gulf States, also Arkansas and Illinois.

\section{Plethodon ( $G E N U S)$}

Tschudr, Mém. Soc. Sci. Nat. Neuchâtel, 1838, p. 92 TYPE: cinereus

Plethodon aeneus Cope and Packard Plcthodon aeneus COPE and Packard

Amer. Nat., Vol. 15, 1881, p. 878.

Plethodon aeneus Cope

Bull. U.S. Nat. Mus., No. 34, 1889, p. 143, fig. 32.

TYPE LOCALITY: Nickajack Cave, southern Tennessee.

RANGE: Virginia to northern Georgia and Tennessee.

\section{Plethodon croceater COPE}

Plethodon croceatcr Cope

Proc. Ac. Nat. Sci. Phila., 1867, p. 210.

Plethodon croceater COPE

Bull. U. S. Nat. Mus., No. 34, 1889, p. 150.

TYPE LOCALITY: Fort Tejon, California (?).

RANGE: Lower California, and California north to El Dorado

County. 


\section{Plethodon dorsalis (COPE) ${ }^{t}$}

Plethodon cinereus dorsalis COPE

Bull. U. S. Nat. Mus., No. 34, 1889, p. 138.

TrPE LOCALITY: Salem, Massachusetts (\%), and Louisville, Kentucky.

RANGE: Southern Illinois, southern Ohio, southern Indiana and western Kentucky.

\section{Plethodon elongatus VAN Denburgh}

\section{Plethodon elongatus VAN DeNBUrgh}

Proc. California Ac. Sci., Ser. 4, Vol. 6, No. ‘. May 12, 1916, p. 216.

TyPe locality: Requa, Del Norte Co., California.

RANGE: Requa, Del Norte Co., California.

\section{Plethodon cinereus (GREeN) ${ }^{2}$}

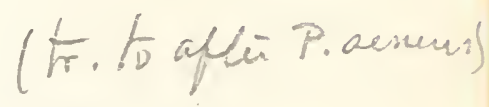

REDBACKED SALAMANDER; DLSKY SALAMANDER (NMES FOR THE

TWO COLOR PHASES)

Salamandra cinerea Green

Journ. Ac. Nat. Sci. Phila., Vol. 1, Pt. ‥ Sept. 1818, p. 356.

Plethodon cinereus Tschudi

Mém. Soc. Sci. Nat. Neuchâtel, 1838, p. 92.

Plethodon cinereus COPE

Bull. U. S. Nat. Mus., No. 34, 1889, p. 133, fig. 30.

TYPE LOCALITY: New York in rocky situations.

RANGE: Eastern Canada and the states east of the Mississippi

River, also Missouri.

\section{Plethodon eschscholtzii (GRAY)}

Ensatina eschscholtzii Gray

Cat. Batr. Grad. Brit. Mus., 1850, p. 48.

Plethodon eschscholtzii Grinnell and CAMP

Univ. California Publ. Zoöl., Vol. 17. July 11, 191\%. p. 132.

Plethodon oregonensis COPE

Bull. U. S. Nat. Mus., No. 34, 1889, p. 148, fig. 35.

TYPE LOCALITY: California.

RANGE: Puget Sound to Los Angeles, California.

1 There is no evidence that Baird ever described this spccies which is generally credited to him. Cope (Proc. Ac. Nat. Sci. Phila., 1869, p. 100) mentions it as a variety of Plethodon cinercus, thus, "Var. $\gamma$. dorsalis, Baird, M.S. 3776. Louisville, Ky.; Salem, Mass." This reference is a nomen nudum pure and simple.

2 Green described this species and also named the synonymous erythronotus upon the same page. It therefore remained for the first reviser to fix the correct name for the species. This Tschudi did in 1838 , 


\section{Plethodon glutinosus (GreeN)}

\section{SLIMY SALAMANDER}

\section{Salamandra glutinosa Green}

Journ. Ac. Nat. Sci. Phila., Vol. 1, Pt. 2, Sept. 1818, p. $35 \%$.

Plethodon glutinosus TschUd

Mém. Soc. Sci. Nat. Neuchâte!, 1828, p. 92.

Plethodon glutinosus CoPE

Bull. U. S. Nat. Mus., No. 34, 1889, p. 139, fig. 31.

TYPE LOCALITY: Not mentioned but obviously Princeton, New

Jersey. Here Green lived, and he mentions often finding the creature under stones.

RANGE: Eastern Canada, New England to Wisconsin, south to Florida, the Gulf States to Texas and to Missouri.

\section{Plethodon intermedius BAIRD}

Plethodon intermedius BAlRD

Proc. Ac. Nat. Sci. Phila., 1867, p. 209.

Plethodon intermedius Cope ${ }^{1}$

Bull. U. S. Nat. Mus., No. 34, 1889, p. 145, fig. 33.

TYPE LOCALITY: Fort Tejon, California (?).

RANGE : Oregon, Washington, Vancouver Island.

\section{Plethodon jordani Blatchley}

Plethodon jordani BLA TCHLEY

25 Ann. Rep. Dept. Geol. Indiana, 1900 (1901), p. 762.

тyPe Locality: Mt. Collins and Indian Pass, 3000-5000 feet altitude, Sevier Co., 'Tenn.

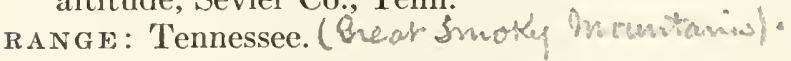

\section{Plethodon metcalfi Brimley}

Plethodon metcalfi BRIMLeY

Proc. Biol. Soc. Washington, Vol. 25, Dec. 4, 1912, p. 138. pl. 6, figs.

$1,2,4,7-11$.

TrPe Locality: Sunburst, Haywood Co., North Carolina. RANGE: Mountains of North Carolina, tane Sertikes sus Vinginia.

Plethodon shermani STEJNEGER

Plethodon shermani STEJNEGER

Proc. U. S. Nat. Mus., Vol. 30, May 22, 1906, p. 559, fig. 1.

TYPE LOCALITy: ${ }^{2}$ Wayah Bald Mountain, North Carolina. RANGE: North Carolina to Georgia.

1 The young of this species (in one of two color phases?) has appeared frcquently in literature as Plethodon crassulus Cope, Proc. Amcr. Philos. Soc., Vol. 23, 1886, p.521; Bull. U.S. Nat. Mus., No.34, 1889 , p. 147, fig. 34

2 Originally given as Nantahala Mt. between Andrews and Aquonfe, North Carolina (in errore).

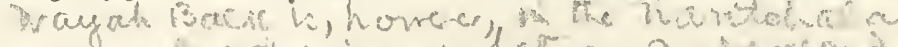






\section{Plethodon vandykei VAN Denzurgu}

Plethodon vandylei VAx Dexibergul

Proc. California Ac. Sci., Ser. 3, Zoöl. Vol. 4, March 14, 1906, p. 61. TyPe locality: Paradise Valley, Mt. Ranier Park, Washington.

RANGE: Washington. (Known from type locality only).

Plethodon wehrlei Fowler and Dunx

Plethodon wehrlei Fowler and Duxy

Proc. Ae. Nat. Sci. Phila., Vol. 69, 1917, p. 23, pl. 4. type Locality: Two Lick Hills, Indiana Co., Pennsylvania. range: Central and western Pennsylvania.

\section{Plethodon yonahlossee DUNN}

Plethodon yonahlossee Dexi

Bull. Amer. Mus. Nat. Hist., New York, Vol. 37, Oct. 13, 1917, p. 598 .

ty pe locality: Near the Yonahlossee Road, about $1_{2}^{1}$ miles

from Linville, North Carolina, altitude 4200 feet.

RANGE: Wooded mountains, near Linville, North Carolina.are $\mathrm{m}$ southaraten Vigines.

Stereochilus (GENUS)

Cope, Proc. Ac. Nat. Sci. Phila., 1869, p. 100

TYPE: marginatus

Stereochilus marginatus (HALLowELL)

Pseudotriton marginatus HaLLowell

Proc. Ac. Nat. Sci. Phila., 1856, p. 130.

Stereochilus marginatum CopE

Proc. Ac. Nat. Sci. Phila., 1869, p. 101.

Stercochilus marginatus COPE

Bull. U.S. Nat. Mus., No. 34, 1889, p. 152, fig. 36.

TYPE LOCALITY: Liberty Co., Georgia.

RANGE: North Carolina to Georgia.

$\checkmark$ ingivias

Gyrinophilus ( $G E$ EN $U S$ )

Cope, Proe. Ac. Nat. Sci. Phila., 1869, p. 108

TYPE: porphyriticus

- Gyrinophilus danielsi (Blatciler)

Spelerpes danielsi Blatchley

25 Ann. Rep. Dept. Geol. Indiana, 1900, (1901), p. 760.

Gyrinophilus danielsi Fowler and Duxx

Proc. Ac. Nat. Sci. Phila.. Vol. 69. 191\%, p. 19. 
type Locality: Mt. Collins and Indian Pass, Sevier Co.,

Tennessee.

RANGE: North Carolina to Georgia, Tennessee.

Gyrinophilus porphyriticus (GRees)

Salamandra porphyritica GreEN

Contr. Maclur. Lyc., Vol. 1, 1827, p. 3, pl. 1, fig. 2.

Gyrinophilus porphyriticus COPE

Proc. Ac. Nat. Sci. Phila., 1869, p. 108.

Gyrinophilus porphyriticus CopE

Bull. U. S. Nat. Mus., No. 34, 1889, p. 155, fig. 37.

TYPE LOCALITY: French Creek near Meadville, Crawford County, Pennsylvania.

RANGE: Southern Canada, southward through the eastern states to Kentucky; Ohio.

\section{Manculus ( $G E N U S)$}

Cope, Proc. Ac. Nat. Sci. Phila., 1869, p. 101

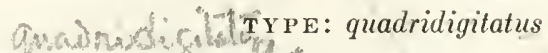

Manculus quadridigitatus (HoLBrook)

DWARF SALAMANDER

Salamandra quadridigitata HoLBrooK

N. Amer. Herp., Ed. 2, Vol. 5, 1842, p. 65, pl. 21.

Manculus quadridigitatus COPE

Proc. Ac. Nat. Sci. Phila., 1869, p. 101.

Manculus quadridigitatus Cope

Bull. U. S. Nat. Mus., No. 34, 1889, p. 159, fig. 38.

TYPE LOCALITY: South Carolina, Georgia and Florida.

RANGE: North Carolina to Florida, Gulf States to Texas.

Manculus remifer CoPE

Manculus remifer COPE

Rep. Peabody Ac. Salem, 1869-70 (1871), p. 84.

Manculus remifer Cope

Bull. U. S. Nat. Mus., No. 34, 1889, p. 158.

TYPE LOCALITY: Jacksonville, Florida.

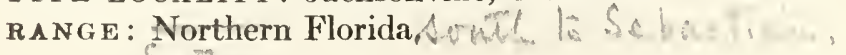
Eaciom

\section{Eurycea $(G E N U S)$}

Rafinesque, Kentucky Gazette, Lexington, N. S.. Vol. 1, No. 9, Feb. 28, 1822, p. 3, column 6

Eurycea bislineata (GreeN) TY PE: lucifuga

Salamandra bislineata GreEN

Journ. Ac. Nat. Sci. Phila., Vol. 1, Sept. 1818, p. 352. 
Enrycea bislineala cirregem (aren) Spersenes cirrigena been.

Jons. ac. hat, Sci. Phila, hel. b, 1839 p. 253

Eurycea tis liusali cirriger. I Imm.

Proc, Biol, Suc, Wrash, bol $33,1920,1,135$.

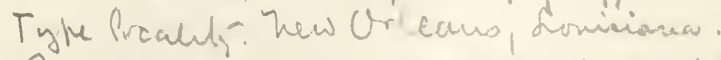

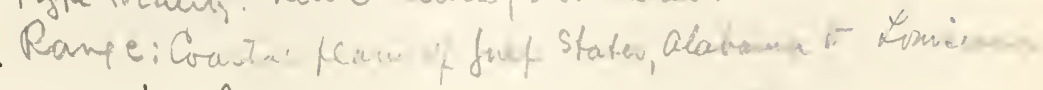

Eyrusea bis hinealà wil derae Dum.

Eurycea bislineatà rilderae Dimn.

Proc. Bicl. Soc. Wash., tol. 33, Dec. 30, 19ro, p.134

Type healify: While Toplitt. Virgmia, too ofect.

Rampe: The hightards of A.ta, R.C., S C., Da., Tenu 


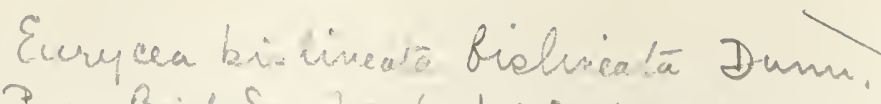
Prre. Baial Sve. Hrark, be 33,1920,10,134 
Spelerpes bilineatus Cope

Bull. U. S. Nat. Mus., No. 34, 1889, p. 163, fig. 40.

TYPE LOCALITY : Western Pennsylvania.

RANGE: Northern New England to Florida, Southern Canada, Lake Superior to Louisiana.

\section{Eurycea gutto-lineata (HoLbiook)}

Salamandra gutto-lineata HoLmiook

N. Amer. Herp., Ed. 1, Vol. 2, 18\%s, p. 61, pl. 12.

Spelerpes guttolineatus $\mathrm{COPE}$

Bull. U.S. Nat. Mus., No. 34, 1889, p. 170, fig. 42.

TYPE LOCALITY: "Carolina, in the middle country."

RANGE: Virginia to Georgia, west to Alabama, Mississippi and

Louisiana.

\section{Eurycea longicauda (Green)}

Salamandra longicauda Green

Journ. Ac. Nat. Sci. Phila., Vol. 1, Sept., 1818, p. 351.

Spelerpes longicaudus Cope

Bull. U.S. Nat. Mus., No. 34, 1889, p. 168, fig. 41.

TyPe locality : New Jersey (probably near Princeton).

RANGE: Southern New England to Florida and Louisiana, west

to Kentucky, Ohio, Tennesee, Illinois and Missouri.

\section{Eurycea lucifuga Rafinesque}

Eurycea lucifuga Rafinesque

Kentucky Gazette, Lexington, (N. S.), Vol. 1, No. 9, Feb. 28, 1822,

p. 3, column 6 .

Gyrinophilus maculicaudus COPE

Amer. Nat., Vol. 94, 1890, p. 967.

TYPE LOCALITy : "Caves near Lexington, Kentueky." RANGE: West Virginia to Tennessee and Kentucky, also Indiana,

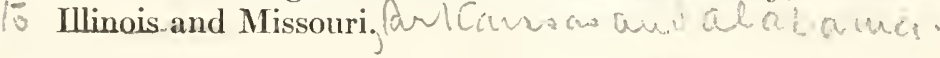

\section{Eurycea melanopleura (COPE)}

Spelerpes melanopleurus Cope

Proc. Ac. Nat. Sci. Phila., 1893, p. 383.

тy pe Locality : Raley's Creek, White River, Missouri.

RANGE: Missouri, arkenen. wer Tewan.

Eurycea montana (BAIRD)

Pseudotriton montanus BAIRD

Journ. Ac. Nat. Sci. Phila., Ser. 2, Vol. 1. 1849, p. 293.

Spelerpes ruber montanus COPE

Bull. U. S. Nat. Mus., No. 34, 1889, p. 179. 
TyPe LOCALity: South Mountain, near Carlisle, Penn.

RANGE: Mountains-from Pennsylvania southward to the Caro-

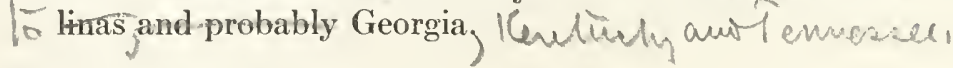

Eurycea multiplicata (COPE)

Spelerpes multiplicatus Cope

Proc. Ac. Nat. Sci. Phila., 1869, p. 106.

Spelerpes multiplicatus COPE

Bull. U. S. Nat. Mus., No. 34, 1889, p. 169, fig. 39.

TYPE LOCALITY: Red River, in Eastern Oklahoma.

RANGE: Missouri and Kansas, through Arkansas to Oklahoma, and New Mexico.

Eurycea platycephala (CAmP)

Spelerpes platycephalus CAMP

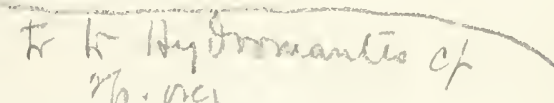

Univ. California Publ. Zoöl., Vol. 17, Sept. 18, 1916, p. 11.

TYPE LOCALITY : Head of Lyell Canon, The Yosemite Nat. Park,

California, 10,800 feet alt.

range: Head of Lyell Canon, The Yosemite National Park.

California, 10,800 feet alt.

Pasedotrition

Euryeer rubra rubra (SonNinI)

RED SALAMANDER

Salamandra rubra SonNini

in Latreille, Hist. Nat. Rept., Vol. 4. 1809, p. 305.

Spelerpes ruber COPE

Bull. U. S. Nat. Mus., No. 34, 1889, p. 172, fig. 43.

TYPE LOCALITY: United States.

RANGE: From New England to Florida westward to Louisiana,

Tennessee, IVisconsin, Illinois, and Ohio.

\section{Eurycea rubra schencki (BRIMLEY)}

Spelerpes ruber schenchi BRIuLEY

Proc. Biol. Soc. Wushington, Vol. 25, Dec. 4, 1912, p. 139, pl. 7,

figs. $12,13,15,17,18,20-29$.

TYPE LOCA LITY : Sunburst, North Carolina, 3,200 feet.

RANGE: Mountains of North Carolina.

\section{Eurycea stejnegeri (FigenuanN)}

Spelerpes stejnegeri EigENMANN

'Trans. Amer. Micr. Soc., Vol. 29,1901 , p. 189, pl. 28.

TYPE LOCALITY: Rock House Cave, Missouri.

RANGE: The Ozark Plateau. 
Precuctoticion (Genus)

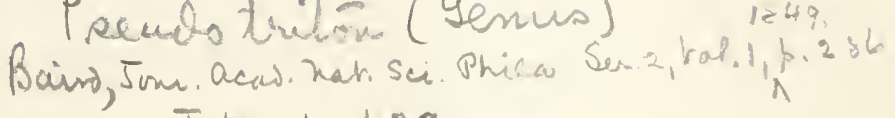
Tyle: rubnar.

$\checkmark$ Pseudotriton rubar nitidno Dum.

Psecubtritor rubra nitions Dum.

Proc. Biol. Soc. Trask., Vol. 33, Dec. 30,1920, f. 133 .

Type localit5: White Top. hit. Yuginia. Huoft.

Rame: Area bounded by the Stone tron hits to the trest, The Blue Ridge to the Sart, the Black hist. If the soutt,

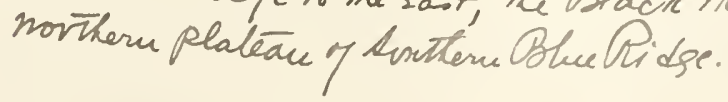

Psecobotritón montanusflarizsonum Hallormele.

Pseniotriton flavisaimon tolumede.

Proc, acai, hair, Sei. Phica, 1856, p.130

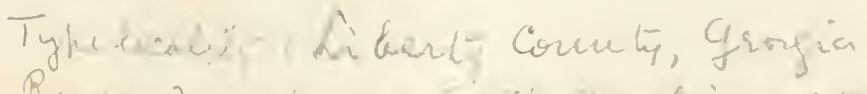

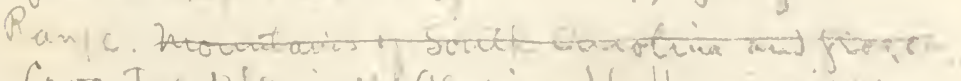

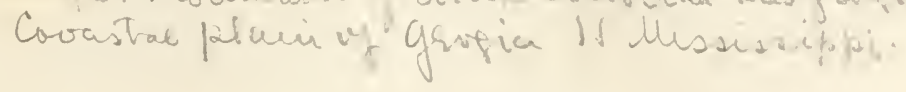


Hydromantes (Derus)

gist $\varepsilon l$, hating, 184.8, $\$ . \times 1$.

Type:genei

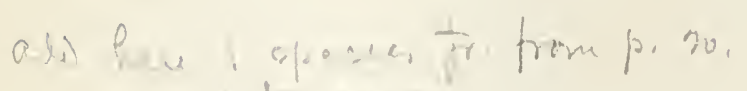
platyugate-

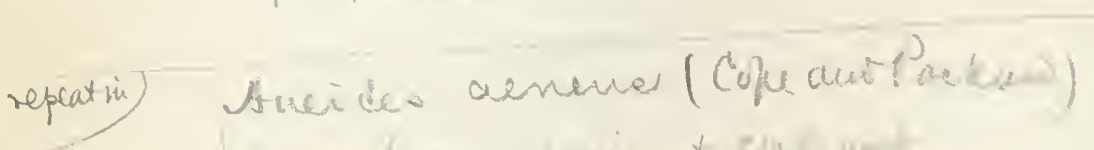

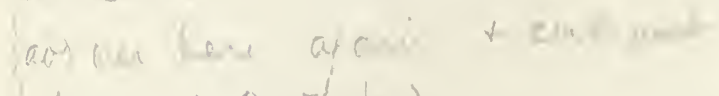
trma in Pucthodin) 


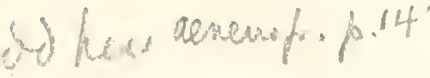

Aneides ${ }^{1}$ (GENUS)

Aneides ferreus Cope

Baird, Iconogr. Encycl., Vol. , 1849, Zoöl., p. 257

TYPE: lugubris

Anaides ferreus $\mathrm{Cope}$

Proc. Ac. Nat. Sci. Phila., 1869, p. 109.

Autodax ferreus COPE

Bull. U. S. Nat. Mus., No. 34, 1889, p. 185, fig. 45.

TyPE LOCALity: Fort Umpqua, Oregon.

RANGE: Northern California, Oregon.

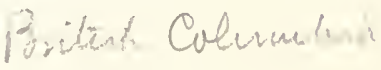

\section{Aneides iëcanus (Cope)}

Plethodon iëcanus Cope

Proc. Ac. Nat. Sci. Phila., 1893, p. 94.

Aneides iëcanus Grinnell and $\mathrm{C}_{\mathrm{A}} \mathrm{p}$

Univ. Calif. Publ. Zoöl., Vol. 17, July 11, 1917, p. 135.

Autodax iëcanus CopE

Bull. U.S. Nat. Mus., No. 34, 1889, p. 187, fig. 46.

ty ye locality : Shasta Co., California.

RANGE: Northern California.

\section{Aneides lugubris lugubris (HALLOWELL)}

Salamandra lugubris Hallowell

Proc. Ac. Nat. Sci. Phila., Vol. 4, 1849, p. 126.

Aneides lugubris BaIRD

Iconogr. Encycl., Vol. 2, 1849, Zoöl., p. 257.

Autodax lugubris CoPe

Bull. U. S. Nat. Mus., No. 34, 1889, p. 183, fig. 4 t.

TYPE LOCA L I Y : "Island of Maui, one of the-Sandwich Islands:" RANGE: California; the Coronados Islands, Mexico.

Aneides lugubris iarallonensis (VAN DenBURGH)

Autodar lugubris farallonensis VAN Denburgir

Proc. Cal. Ac. Sci., Ser. 3, Zoöl., Vol. 4, 1905, p. 5, pl. \&.

Aneides lugubris farallonensis GRINnELL and CAMP

Univ. Calif. Publ. Zoöl., Vol. 17, July 11, 1917, p. 135.

TYPE LOCALITY: South Farallon Island, California. RANGE: The Farallon Islands.

1 Generic name spelt also Anaides (p. 460 and \%oöl. p. 256) in same reference - but Anaides is preoc. by Anaides of Westwood, Proc. Ent. Soc. Lond., 1841, for a genus of Longicorn beetles. Aneides seems to have been the spelling intended. 


\section{Desmognathus ( $G E N U S$ )}

BAIrD, Journ. Ac. Nat. Sci. Phila., Ser. 2, Vol. 1, 1849, pp. 282, 285 TYPE: fuscus

Desmognathus brimleyorum STE.JNEGER

Desmognathus brimleyorum STEJNEGER

Proc. U. S. Nat. Mus., Vol. 17, May 11, 1895, p. 597.

TyPe L OCAlity: Hot Springs, Arkansas.

RANGE: Arkansas ount for as

\section{Desmognathus fuscus fuscus (RAfinesque)}

Triturus fuscus RAFInESQUE

Ann. Nat., Lexington, No. 1, March 2Q, 1820, p. 4.

Desmognathus fuscus BAIRD

Journ. Ac. Nat. Sci. Phila., Ser. 2, Vol. 1, 1849, p. 285.

Desmognathus fusca fusca CoPE

Bull. U. S. Nat. Mus., No. 34, 1889, p. 194, fig. 48.

TYPE LOCALITY: Northern parts of State of New York.

RANGE : Southern Canada to the mountains of the Carolinas and

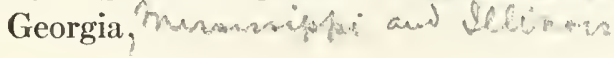

\section{Desmognathus fuscus auriculatus (HoLBRook)}

Salamandra auriculata HoLBrook

N. Amer. Herp., Ed. 1, Vol. 3, 1838, p. 115, pl. 28.

Desmognathus auriculatus BAIRD

Journ. Ac. Nat. Sci. Phila., Ser. 2, Vol. 1, 1849, p. 286.

Desmognathus fusca auriculata CoPE

Bull. U. S. Nat. Mus., No. 34, 1889, p. 195, fig. 49.

TYPE LOCALITY : Riceborough, Georgia.

RANGE: Lowlands from Virginia to Florida and the Gulf States.

\section{Desmognathus monticola DUNN}

Desmognathus monticola DUNN

Proc. Biol. Soc. Washington, Vol. 29, April 4, 1916, p. 73.

TYPE LOCALITY: Elk Lodge, near Brevard, North Carolina.

RANGE: From Virginia and West-Virginia to Georgia. (In the mountains 500-4500 feet.)

\section{Desmognathus ochrophaeus ochrophaeus (COPE)}

Desmognathus ochrophaea Cope

Proc. Ac. Nat. Sci. Phila., 1859, p. 124.

Desmognathus ochrophaea CoPE

Bull., U.S. Nat. Mus., No., 34, 1889, p. 191, fig. 47. 


TYPe Locality: Susquehanna Co., Penn.

RANGE: New Brunswick and Northern New York soutl to the mountains of Maryland.

Desmognathus ochrophaeus carolinensis DUNN

Desmognathus ochrophaeus carolinensis DUNN

Proc. Biol. Soc. Washington, Vol. 99, April 4, 1916, p. 74.

TyPE LOCAlity: Mi. Mitchell, North Carolina, over 6500 feet. RANGE: West Virginia to Georgia (in the mountains).

Desmognathus quadra-maculatus (НоLrRook)

Salamandra quadra-maculata НоцвRоок

N. Amer. Herp., Ed. 1, Vol. 4, 1840, pl. 2\%. (The text p. 121 reads maculo-quadrata.)

Desmognathus quadrimaculata SteJNeger

Proc. U. S. Nat. Mus., Vol. 26, Jan. 29, 1903, p. 557.

TYPE LOCALITY: Georgia and the Carolinas.

RANGE: Virginia to Georgia, in the mountains, $2000-6000$ feet.

\section{Leurognathus ( $G E N U S)$}

Moore, Proc. Ac. Nat. Sci. Phila., 1899, p. 316

TYPE: marmoratì

Leurognathus marmoratis Moore

Leurognathus marmorat is MoORE

Proc. Ac. Nat. Sci. Phila., 1899, p. 316.

TyPE LOCALITy: South slope Grandfather Mt., North Carolina, 3500 feet.

RANGE: Mountains of North Carolina.

min

Typhlotriton ( $G E N U S)$

Stejneger, Proc. U.S. Nat. Mus., Vol. 1j, 1892, p. 115

TYPE: spelaens

Typhlotriton spelaeus STEJNEGER

Typhlotriton spelaeus STEJNeger

Proc. U. S. Nat. Mus., Vol. 15, Aug. \&, 1892, p. 115, pl. 9.

TYPE LOCALITY: Rock House Cave, Missouri.

RANGE: Rock House Cave, Barry Co., Marble Cave, Stone Co.,

Doris Cave, Wright Cave, Missouri. 


\section{MEANTES (SUBORDER)}

Linné, Syst. Nat., Ed. 12, Vol. 1, Pt. 2, 1766, sign.

Rrrr 5, Addenda (not paged)

\section{SIRENIDAE (FAMILY)}

Siren $(G E N U S)$

Linné, Syst. Nat., Ed. 12, Vol. 1, pt. \&, 1766. sign.

Rrrr 5, Addenda (not paged)

Siren lacertina LinNé

TY PE: lacertina

MUD-EEL

Siren lacertina LinNÉ

Syst. Nat., Ed. 12, Vol. 1, pt. 2, 1766, sign. Rrrr 5, Addenda (not paged).

Siren lacertina Cope

Bull. U. S. Nat. Mus., No. 34, 1889, p. 226, fig. 57.

TYPE LOCALITY: "Habitat in Carolinae paludosis."

RANGE: Eastern Virginia to Florida, through the Gulf States to

the west side of the Rio Grande. Ascends the Mississippi Val-

ley to Illinois and Indiana. Occurs in scattered localities in Arkansas and Missouri.

\section{Pseudobranchus ( $G E N U S$ )}

Gray, Ann. Philos. (N. S.), Vol. 10, 1825, p. 216

TYPE : striatus

\section{Pseudobranchus striatus (Le Conte)}

Siren striata Li CoN'te

Ann. Lyc. New York, Vol. 1, pt. 1, 1824, p. 53, pl. 4.

Pseudobranchus striatus Gray

Ann. Philos., (N. S.), Vol. 10, 1825, p. 216.

Pseudobranchus striatus Cope

Bull. U. S. Nat. Mus., No. 34, 1889, p. 230, fig. 58.

TYPE LOCALITY: Not given, but by inference it was one of the

Le Conte plantations in Floyd or Liberty Co., Georgia.

RANGE: Swamps of southern South Carolina, lower Georgia and Florida. 




\section{SALIENTIA (ORDER) \\ Laurenti, Syn. Rept., 1768, p. 跳 \\ COSTATA (SUBORDER)}

Lataste, Act. Soc. Linn. Bordeaux, Vol. 33, 1879, p. 339

DISCOGLOSSIDAE (FAMILY)

Ascaphus (GENUS)

Stejneger, Proc. U.S. Nat. Mus., Vol. Q1, June 20,1899 , p. 899 TYPE : truei

Ascaphus truei SteJNeger

Ascaphus truei Stejneger

Proc. U. S. Nat. Mus., Vol. 21, June 20, 1899, p. 900, figs. 1-4, pl. 89. TYPE locality: Humptulips, Washington. RANGe: Chehalis Co. and Pierce Co., Washington,

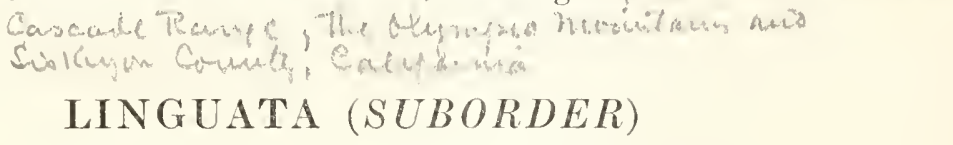

Gravenhorst, Thierreich, 1845, p. 43

SCAPHIOPODIDAE ( $F A M I L Y)$

Scaphiopus (GENUS)

Ноцвrook, N. Amer. Herp., Ed. 1, Vol. 1, 1836, p. 85 TYPE: solitarius $=$ holbrookii

Scaphiopus couchii BAIRD

Scaphiopus courhii BAIRD

Proc. Ac. Nat. Sci. Phila.. Vol. 7, 185k, p. 69.

Scaphiopus courhii Cope

Bul!. U. S. Nat. Mus., Vol. 34, 1882, p. 301, fig. 75.

ty e locality: Rio Nasas, Coahuila and Matamoros, Tamaulipas, Mexico.

RANGE: Texas to Arizona, northern states of Mexico, Lower California.

Scaphiopus hammondii BAIRD

Scaphiopus Hammondii BalRD

Rep. Pacif. R. R. Surv., Vol. 10, Pt. 4, 1875, p. 12, pl. 28, fig. 2. 
Spea hammondii CoPE

Bull. U.S. Nat. Mus., No. 34, 1889, p. 303, fig. 77.

TYPE LOCALITY: Fort Reading, California.

RANGE: Western and southwestern states from Montana south

to Texas and Mexico, and westward to the Pacific Coast states,

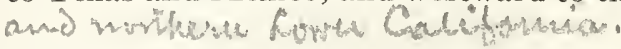

Scaphiopus holbrookii holbrookii ${ }^{1}$ (HARLAN)

SPADEFOOT

Rana holbrooliii IIARLAN

Med. Phys. Researches, 1835, p. 105.

Scaphiopus holbrookii COPE

Bull. U. S. Nat. Mus., No. 34, 1889, p. 298.

TYPE LOCALITY: South Carolina.

RANGE: Eastern states, Massachusetts to Florida, west to Louisiana, Texas to Arkansas.

\section{Scaphiopus holbrookii albus (GARmaN)}

Scaphiopus albus GARMAN

Proc. Amer. Ass. Adv. Sci., Buffalo, 1877, p. 194.

TYPE LOCALITy: Key West, Florida.

RANGE: Florida Keys and possibly the extreme southern part of

the peninsula of Florida.

\section{Scaphiopus hurterii STRECKER}

Scaphiopus hurterii STRECKER

Proc. Biol. Soc. Washington, Vol. 23, July 23, 1910, p. 116, pl. 2, figs. 3-4.

TYPE LOCALity: Waco, Texas.

RANGE: Texas.

BUFONIDAE (FAMILY)

Bufo ( $G E N U S)$

Laurentr, Syn. Rept., 1768, p. 25

TYPE: vulgaris $=$ bufo

Bufo alvarius Girard

Bufo alvarius Girard

U. S. Mex. Bound. Surv., Vol. 2, 1859, Rept., p. 26, pl. 41, figs. 1-6.

Bufo alvarius Cope

Bull. U. S. Nat. Mus., No. 34, 1889, p. 265 , fig. 62.

1 This species usually appears in the literature as Scaphiopus solitarius Holbrook. Harlan's description appeared a year before Holbrook's but Harlan knew of Holbrook's discovery of tbe species for he writes (I. c.) "Tbis new species, first figured and described by Dr. Holbrook (vide his valuable work on North Ameriean Reptiles), possesses very peculiar charaeters. . . ." 


TYPE LOCALity: Valley of the Gila and Colorado Rivers. RANGE: Southern Arizona and Southern California.

Bufo americanus HoLBROoK

AMERICAN TOAD

Bufo americanus HoLBRook

N. Amer. Herp., Ed. 1, Vol. 1. 1836, p. 75, pl. 2.

Bufo lentiginosus americanus $\mathrm{CopE}$

Bull. U. S. Nat. Mus., No. 34, 1889, p. 284. fig. 70.

TYPE LOCALITY: "From Maine through all the Atlantic States." RANGE: Eastern North America from Hudson Bay Southward.

Bufo boreas boreas (Baird and Girard)

Bufo boreas BAIRD and GIRARD

Proc. Ac. Nat. Sci. Phila., Vol. 6, 1852, p. 174.

Bufo columbiensis COPE

Bull. U. S. Nat. Mus., No. 34, 1889, p. 267 , fig. 63.

TYPE locality: Columbia River and Puget Sound.

RANGE: Colorado north and west to Puget Sound. Also British

Columbia and southwestern Alaska to Prince William Sound.

Bufo boreas halophilus (BAIRD and Girard)

Bufo halophila BAIRD and GIRARD

Proc. Ac. Nat. Sci. Phila., Vol. 6. 1853, p. 301.

Bufo boreas halophilus CAup

Univ. California Publ. Zoöl., Vol. 17, No. 9, Feb. 3, 1917, p. 116.

Bufo columbiensis halophilus COPE

Bull. U. S. Nat. Mus., No. 34, 1889, p. 269, fig. 64.

TYPE LOCALITY: Benicia, California.

RANGE: Western Nevada, California, and northern Lower Califormia.

Bufo canorus CANIP

Bufo canorus CanlP

Univ. California Publ. Zoöl., Vol. 17, Nov. 17, 1916, p. 59.

trpe locality : Porcupine Flat, Yosemite National Park, California.

RANGE: Region about Porcupine Flat, Yosemite National Park, California.

\section{Bufo cognatus cognatus (SAY)}

Bufo cognatus $\mathrm{S}_{\mathrm{AY}}$

Long's Exp. Rocky MIts., Vol. , 18\&3, p. 190.

Bufo cognatus Cope

Bull. U. S. Nat. Mus., No. 34, 1889, p. 275, fig. 67. 
rleters w

TyPe locality: Arkansas River, Prowers Co., Colorado.

RANGE: Arizonayand Colorado to Texas, Arkansas to Kansas,

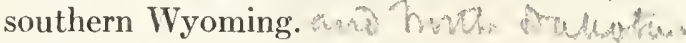

Bufo cognatus californicus CAMP

Bufo cognatus califormicus CAMP

Univ. California Publ. Zoöl., Vol. 12, April 2, 1915, p. 331.

Ty PE LOCAlity: Sta. Paula, Ventura Co., California.

RANGE: Southwestern California.

Bufo compactilis WiegmanN

Bufo compactilis WiegManN

Isis, 1833 , p. 661.

Bufo compactilis COPE

Bull. U. S. Nat. Mus., No. 34, 1889, p. 279, fig. 65.

TyPE LOCAlity: Mexico.

RANGE: Northern Mexico, Texas, New Mexico and Arizona.

Bufo debilis Girard

Bufo debilis GIRARD

Proc. Ac. Nat. Sci. Phila., Vol. 7, 1854, p. 87.

Bufo debilis Cope

Bull. U. S. Nat. Mus., No. 34, 1889, p. 264, fig. 61.

TYPE LOCALITY: Lower part of the valley of the Rio Grande,

Texas and in Tamaulipas, Mexico.

RANGE: Colorado south into the northern states of Mexico.

- Bufo fowleri Garman

FOWLER'S TOAD

Bufo fowleri Garman

Bull. Essex Inst. Vol. 16, 1884, p. 42.

Bufo lentiginosus fowleri COPE

Bull. U. S. Nat. Mus., No. 3t, 1889, p. 279, fig. 68.

TYPE LOCALITY: "Manitoba to Winnipeg; Massachusetts." RANGE: New England and New York, southward to Georgia,

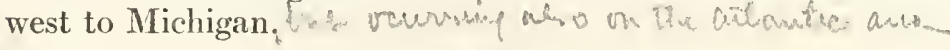

Bufo hemiophrys Cope

Bufo hemiophrys Cope

Proc. Amer. Philos. Soc., Vol. 23, 1886, p. 515.

Bufo hemiophrys Cope

Bull. U. S. Nat. Mus., No. 34, 1889, p. 273 , fig. 66.

type locality: Pembina and Turtle Mountains, North Da-

kota.

RANGE: North Dakota and Manitoba. 


Bufo punctatus BAIRD and GIrarid

Bufo punctatus Baird and Girard

Proc. Ac. Nat. Sci. Phila., Vol. 6, 1852, p. 173.

Bufo punctatus COPE

Bull. U.S. Nat. Mus., No. 34. 1889, p. 262, fig. 60.

TyPE Locality: Rio San Pedro, tributary of the Rio Grande del Norte, Texas.

RANGE: Western Texas, Arizona, Utah, Southern California and Lower California.

Bufo quercicus HoLBrook

Bufo qucrcicus HoLBRoок

N. Amer. Herp., Ed. 1, Vol. 4, 1840, p. 109, pl. 92.

Bufo quercicus COPE

Bull. U. S. Nat. Mus., No. 34, 1889, p. 291, fig. 79.

TYPE LOCALITY: Charleston, South Carolina and Smithville,

North Carolina.

RANGE: Nortil Carolina to Alabama and Florida.

Bufo terrestris BonNaterRe

SOUTHERN TOAD

Bufo terrestris BonNaterRe

Tabl. Encycl. Méth. Erp., 1789, p. 8.

Bufo lentiginosus lentiginosus CoPE

Bull. U. S. Nat. Mus., No. 34, 1889, p. 289. fig, 71.

TYPE LOCALITY : "La Caroline."

RANGE: The Carolinas to Florida, west to the Mississippi.

Bufo valliceps WiegmanN

Bufo ralliceps WiegmanN

Isis, $1833, \mathrm{p} .65 \%$.

Bufo valliceps COPF

Bull. U. S. Nat. Mus., No. 34, 1889, p. 월, fig. 73.

TYPE LOCALITY: Mexico.

RANGE: Louisiana, Texas south to Costa Rica.

Bufo woodhousii Girard

Bufo woodhonsii Girard

Proc. Ac. Nat. Sci. Phila., Vol. 7, 1854, p. 86.

Bufo lentiginosus woodhousei CopE

Bull. U. S. Nat. Mus., No. 34, 1889, 281, fig. 69.

TyPE LOCAlity: San Francisco Mt., Arizona.

RANGE: Texas to Kansas, Nebraska and Montana, west to Nevada, Arizona and southeastern California. 


\section{HYLIDAE ( FAMILY)}

\section{Acris ( $G E N U S$ )}

Duméril and Bibron, Erp. Gén., Vol. 8, 1841, p. 506 TYPE: gryllus

Acris gryllus (Le Conte)

\section{CRICKET-FROG}

Rana gryllus Le Conte

Ann. Lyc. New York, Vol. 1, Pt. 2, 1825, p. 282.

Acris gryllus Duméril and BıBon

Erp. Gén., Vol. 8, 1841, p. 507.

Acris gryllus Cope

Bull. U. S. Nat. Mus., No. 34, 1889, p. 324, fig. 82.

TYPE locality: Not given.

RANGE: Florida to New York and-Connecticut in the East;

northward through the Central Valley from Louisiana and

Texas to the Canadian Northwest Territories.

\section{Pseudacris (GENUS)}

Fitzinger, Syst. Rept., 18ł3, p. 31

TYPE : nigrita

\section{Pseudacris feriarum (BAIRD)}

Helocaetes feriarum BAIRD

Proc. Ac. Nat. Sci. Phila., Vol. 7, 1854, p. 60.

Pseudacris feriarum CoPE

Proc. Ac. Nat. Sci. Phila., 1862, p. 157.

Chorophilus feriarum COPE

Bull. U. S. Nat. Mus., No. 3\$, 1889, p. 339, fig. 86.

TYPE LOCALity: Carlisle, Pennsylvania.

RANGE : Eastern United States, west to Illinois.

\section{Pseudacris nigrita ( $\mathrm{Le}$ Conte)}

Rana nigrita LE CoNTe

Ann. Lyc. New York, Vol. 1, Pt. 2, 1825, p. 282.

Pseudacris nigrita GÜNTHER

Cat. Batr. Sal. Brit. Mus., 1858, p. 97.

Chorophilus nigritus COPE

Bull. U. S. Nat. Mus., No. 34, 1889, p. 397, fig. 85.

TYPE LOCALITY: Not given.

RANGE: South Carolina to Mississippi.

Pseudacris occidentalis (BAIRD and Girard)

Litoria occidentalis BAIRD and GirarD

Proc. Ac. Nat. Sci. Phila., Vol. 6, 1853, p. 301. 


Chorophilus occidentalis Cope

Bull. U. S. Nat. Mus., No. 34, 1889, p. 335, fig. 84.

TYPE LOCALITY: The type was incorrectly stated to have come

from San Francisco.

RANGE: Georgia and Florida, west to Arkansas and Texas.

\section{Pseudacris ocularis (HoLBrook)}

IIylodes ocularis Howвrook

N. Amer. Herp., Ed. 1, Vol. 3, 1838, p. 79, pl. 14.

Chorophilus ocularis CopE

Bull. U. S. Nat. Mus., No. 34, 1889 , p. 348.

TYPE LOCALITY: South Carolina and Georgia.

RANGE: Southeastern United States.

Pseudacris ornata (HoLbrook)

Rana ornata HoLbrook

N. Amer. Herp., Ed. 1, Vol. 1, 1838, p. 97, pl. 16.

Chorophilus ornatus Cope

Bull. U. S. Nat. Mus., No. 34, 1889, p. 333, fig. 83.

TyPf Locality: Between the Cooper and Ashley Rivers, four

miles from Charleston, South Carolina.

RANGE : South Carolina to Florida, west to Texas.

Pseudacris triseriata (WIED)

Hyla triseriata WIED

Reise Nord-Amer., Vol. 1, Pt. 4, 1838, p. 249.

Chorophilus triseriatus COPE

Bull. U. S. Nat. Mus., No. 34, 1889, p. 349, fig. 87.

TYPE LOCALITY: Mt. Vernon, Ohio River, Indiana.

RANGE: Alleghany Mts. to New Mexico, Arizona and Idaho.

\section{Pseudacris septentrionalis Boulenger}

Chorophilus septentrionalis Boulenger?

Cat. Batr. Sal. Brit. Mus., 1882, p. 335, pl. 23, fig. 1.

TYPE LOCALITY: Great Bear Lake, Canada.

RANGE: Canadian Northwestern swamp areas.

Hyla andersonii BAIRD

$$
\text { Hyla ( } G E N C S \text { ) }
$$

Laurenti, Syn. Rept., 1768, p. 32

TYPE: viridis

ANDERSON TREE-FROG

Hyla andersonii BAIRD

Proc. Ac. Nat. Sci. Phila., Vol. 7, 1854, p. 60. 
IIyla andersonii COPE

Bull. U. S. Nat. Mus., No. 34, 1889, p. 365, fig. 91, pl. 83, fig. 1.

TyPe locality: Anderson, South Carolina.

RANGE: White cedar swamps in Southern New Jersey, and South

Carolina.

Hyla arenicolor Cope

IIyla arcnicolor Cope

Journ. Ac. Nat. Sci. Phila.. Ser. 2, Vol. 6, July, 1866, p. 84.

IIyla arenicolor COPE

Bull. U. S. Nat. Nus., No. 34, 1889, p. 369, fig. 93.

TyPE Locality: Northern Sonora, Mexico.

RANGE: Most of the Southwestern United States from Utah to Lomensouthern California, east to Texas and in Mexico south to

Guadalajara and Toluca.

Hyla baudini Duméril and BibroN

IIyla baudinii DumérIr and BIBRoN

Erp. Gén., Vol. 8, 1841, p. 564.

Smilisca baudinii Cope

Bull. U. S. Nat. Mus., No. 34, 1889, p. 379.

TYPE LOCALITY: Mexico.

RANGE: Central America north to southern Texas.

\section{Hyla cinerea (SCHNEIDER)}

Calamita cinereus SCHNeiner

Hist. Ampl., Vol. 1, 1799, p. 174.

IIyla cinerea GARMAN

Bull. Illiuois State Lab. Nat. Hist., Vol. 3. 1899, p. 349.

IIyla carolinensis COPE

Bull. U. S. Nat. Mus., No. 34, 1889, p. 366, fig. 92.

TYPE LOCALITY: "Inhabits Carolina."

RANGE: Virginia to Florida, west to Texas and northward up the

Mississippi Valley to Southern Illinois.

\section{Hyla crucifer Wien}

SPRING-1PEPER

IIyla crucifer WIED

Reise Nord-Amer., Vol. 1, Pt. 5, 1838, p. 275.

Hyla pickeringii COPE

Bull. U. S. Nat. Mus., No. 34, 1889, p. 352, fig. 88.

TYPE LOCALITY : Cantonment Leavenworth, "Kansas."

RANGE: New Brunswick to Manitoba, soutl to South Carolina,

Louisiana, Arkansas and Kansas. 
Hefla carierea evirata Drma eopreia, ho. 53,191 \& 10.2.1 
Hyla evittata Miller

Hyla evittata MILLER

Proc. Biol. Soc. Washington, Vol. 13, 1899, p. 75.

TYPE locality: Four Mile Run, Virginia (near the City of Washington).

RANGE: Reed beds and lily pools along the Potomac and York Rivers, Virginia, also New Jersey.

Hyla eximia BAIRD

IIyla eximia BAIRD

Proc. Ac. Nat. Sci. Phila., Vol. 7, 1854, p. 61.

IIyla eximia Cope

Bull. U. S. Nat. Mus., No. 34, 1889, p. 361.

TYPE LOCALITY: City of Mexico.

RANGe: Mexico and New Mexico.

\section{Hyla femoralis LATreiluLe}

Ifyla femoralis LATREILLE

Hist. Nat. Rept., Vol. Q, 180\&, p. 181.

Hyla femoralis COPE

Bull. U. S. Nat. Mus., No. 34, 1889, p. 371, fig. 94.

TYPE LOCALITY: Carolina.

RANGE: Carolinas to Florida and west to Texas.

Hyla gratiosa Le Conte

Hyla gratiosa Le Conte

Proc. Ac. Nat. Sci. Phila., 1856, p. 146, pl. 4.

Hyla gratiosa $\mathrm{COPE}$

Bull. U. S. Nat. Mus., No. 34, 1889, p. 37\%, fig. 96, pl. 83, fig. 5.

TYPE LOCALITY : “Lower country of Georgia," evidently one of

the Le Conte plantations in Floyd or Liberty Counties. RANGE: South Carolina to Florida and Mississippi.

Hyla regilla BAIRD and GIRARD

PACIFIC TREE TOAD

IIyla regilla Baird and Girand

Proc. Ac. Nat. Sei. Phila., Vol. 6, 1852, p. 174.

Hyla regilla COPE

Bull. S. Nat. Mus., No. 34, 1889, p. 355, fig. 89.

TyPE LOCALITY: " Sacramento River, in Oregon and Puget Sound."

RANGE: Vancouver Island and British Columbia to Lower California; Nevada. 
Hyla squirella LATreiLle.

IIyla squirella LATREHLLE

Hist. Nat. Rept., Vol. Q, 1809, p. 181.

Hyla squirella Cоре

Bull. U. S. Nat. Mus., No. 34, 1889, p. 363, fig. 90.

TYPE LOCALITY: Carolina.

RANGE: Virginia to Florida, west to Texas, and northward up the Mississippi basin to Indiana.

Hyla versicolor versicolor Le CONTE

THE TREE TOAD, RAIN TOAD

Hyla versicolor LE CONTE

Ann. Lyc. New York, Vol. 1, Pt. 2, 1825, p. 281.

Hyla versicolor Cope

Bull. U. S. Nat. Mus., No. 34, 1889, p. 373, fig. 95.

TYPE LOCALITY: “Northern States."

RANGE: Maine, Southern Canada, west to Minnesota, south to

the Gulf States (Texas and Arkansas in part only).

Hyla versicolor chrysoscelis (COPE)

IIyla femoralis chrysoscelis CopE

Bull. U. S. Nat. Mus., No. 17, 1880, p. 29.

Hyla versicolor chrysoscelis STRECKER

Proc. Biol. Soc. Washington, Vol. 33, 1910, p. $11 \%$.

TYPE LOCALITY: Dallas, Texas.

RANGE: Southern Arkansas to east-central Texas.

\section{LEPTODACTYLIDAE (FAMILY)}

\section{Eleutherodactylus ( $G E N U S)$}

Dunéril and Bıbron, Erp. Gén., Vol. 8, 1841, p. 620

TYPE: martinicensis

Eleutherodactylus latrans (COPE)

Lithodytes latrans COPE

Bull. U. S. Nat. Mus., No. 17, 1880, p. $25 .^{1}$

Lithodytes latrans COPE

Bull. U. S. Nat. Mus., No. 34, 1889, p. 316, fig. 80 .

ty Pe locality : Helotes, Bexar Co., Texas.

RANGE: Texas.

1 Mocquard (Bull. Soc. Philo., Ser. 9, 1, 1899, p. 159) is inelined to believe that this species is a synonym of Eleutherodactylus augusti (Brocchi), but as yet there has been no actual comparison of material and it is perhaps better to keep them apart until such comparison can be made. 


Eleutherodactylus ricordii (DuníriL and BrBRoN)

IIylodes ricordii DuméRIL and Bıron

Erp. Gén., 8, 1841, p. 693.

Eleutherodactylus ricordii STEJNEGER

Shattuck's Bahama Islands, 1905, p. 331.

Lithodytes ricordii COPE

Bull. U. S. Nat. Mus., No. 34, 1889, p. 318.

TYPE LOCALITY: Cuba.

RANGE: Bahama Islands, Cuba and Florida, north to Brevard Co.

(probably accidentally introduced).

\author{
Syrrhophus (GENUS) \\ Cope: Amer. Nat., Vol. 12, 1878, p. 253 \\ TYPE: marnockii
}

\title{
Syrrhophus campi STEJNEGer
}

Syrrhophus campi STEJNEGER

Proc. Biol. Soc. Washington, Vol. 28, June 29, 1915, p. 131.

TYPE LOCALITy: Brownsville, 'Texas.

RANGE: At present known from the type locality only.

\section{Syrrhophus marnockii Cope}

Syrrhophus marnockii CoPE

Amer. Nat., Vol. 12, 1878, p. 253.

Syrrhophus marnochii $\mathrm{COPE}$

Bull. U.S. Nat. Mus., No. 34, 1889, p. 318, fig. 81.

Ty PE locality : near San Antonio, Texas.

RANGE: Known from type locality only.

\section{RANIDAE (FAMILY)}

Rana $(G E N U S)$

Linné, Syst. Nat., Ed. 10, Vol. 1, 1758, p. 210

TYPE: temporaria

Rana areolata BAIRD and GIRARD

Rana arcolata BAIRD and GIRARD

Proc. Ac. Nat. Sci. Phila., Vol. 6, 1859, p. 173.

Rana areolata COPE

Bull. U. S. Nat. Mus., No. 34, 1889, p. 409, fig. 103.

TYPE LOCA LITY : Indianola, Texas.

RANGE: Georgia, Texas, Missouri, Indiana and Illinois. 


\section{Rana aesopus (CoPE)}

\section{GOPHER-FROG}

Rana areolata aesopus Cope

Proc. Amer. Philos. Soc., Vol. 23, Dec. 1886, p. 517.

Rana areolata aesopus Cope

Bull. U. S. Nat. Mus., No. 34, 1889, p. 412.

TYPE LOCALITY : Micanopy, Florida. .

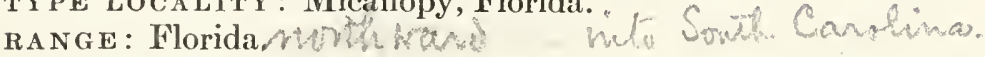

Rana aurora BAIRD and GIRARD

Rana aurora BAIRD and Girard

Proc. Ac. Nat. Sci. Phila., Vol. 6, 1859, p. 174.

Rana agilis aurora GOPE

Bull.U..S. Nat. Mus., No.34, 1889, p. 439, fig.-113.

TYPE LOCAlity: Puget Sound.

RANGE: California to Washington, Vancouver Island.

Rana boylii boylii (BAIRD)

Rana boylii BAIRD

Proc. Ac. Nat. Sci. Phila., Vol. 7, 1854, p. 62.

Rana boylii boylii $\mathrm{C}_{\mathrm{AMP}}$

Univ. California Publ. Zoöl., Vol. 17, No. 9, Feb. 3, 1917, p. 117.

Rana boylii Cope

Bull. U. S. Nat. Mus., No. 34, 1889, p. 444, fig. 115.

type locality: El Dorado Co., California.

RANGE : California and southwestern Oregon.

Rana boylii muscosa CaMP

Rana boylii muscosa $\mathrm{C}_{\mathrm{AMP}}$

Univ. Calif. Publ. Zoöl., Vol. 17, No. 9, Feb. 3, 1917, p. 118.

TYPE L OCAlity : Arroyo Seco Cañon, at about 1300 feet altitude, near Pasadena, California.

RANGe: California: San Gabriel, San Bernadino and San Jacinto Mts.

\section{Rana boylii sierrae CAMP}

Rana boylii sierrae $\mathrm{CAMP}_{\mathrm{A}}$

Univ. Calif. Publ. Zoöl., Vol. 17, No. 9, Feb. 3, 1917, p. 120.

TYPE LOCA Lity : Matlock Lake, Sierra Nevada, Inyo Co., California, 10,500 feet altitude.

RANGE: Southern half of Sierra Nevada, above 7,000 feet altitude, California.

\section{Rana cantabrigensis cantabrigensis (BAIRD)}

Rana cantabrigensis BAIRD

Proc. Ac. Nat. Sci. Phila., Vol. 7, 1854, p. 62. 


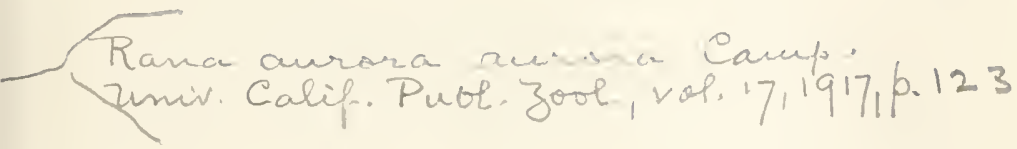



Rana cantabridgensis cantabrigensis $\mathrm{COPE}$

Bull. U. S. Nat. Mus., No. 34, 1889, p. 436, fig. 110. TYPE LOCALITY: Cambridge, Mass. (in errore). RANGE: Illinois and-Mimesota northward in-Ganada to James is Bay-in-the east to Great Slare Lake in the west.

\section{Rana cantabrigensis latiremis COPE}

Rana cantabridgensis latirem is COPE

Proc. Amer. Philos. Soc., Vol. 93, 1886, p. 520.

Rana cantabrigensis latiremis COPE

Bull. U. S. Nat. Mus., No. 34, 1889, p. 435, fig. 111.

TYPE LOCALITY: Lake Alloknagik, Alaska.

RANGE: Alaska, southeastward to the region of Great Slave Lake.

Rana catesbeiana SHAw

BULLFROG

Rana catesbeiana SHaw

Gen. Zool., Vol. 3, Pt. 1, 1802, p. 106, pl. 33.

Rana catesbiana Cope

Bull. U. S. Nat. Mus., No. 34, 1889, p. 424, fig. 108.

TYPE LOCALITY: Shaw evidently knew of the Bull Frog, principally from Catesby, but he only speaks of it as being North American and apparently saw no actual specimens himself.

RANGE: Widespread in North America, east of the Rocky Moun-

tains.

\section{Rana clamitans LATREILLE}

GREEN FROG, SPRING-FROG

Rana clamitans LATREILLE

Hist. Nat. Rept., Vol. ㅇ, 1802, p. 157.

Rana clamata Cope

Bull. U. S. Nat. Mus., No. 34, 1889, p. 419 , pl. 107.

TYPE LOCALITY: Charleston, South Carolina.

RANGE: Eastern North America. Canada to Florida and Louisiana, west to Michigan, Illinois and Arkansas.

Rana draytonii BAIRD and GirARD

Rana draytonii BAIRD and GIRARD

Proc. Ac. Nat. Sci. Phila., Vol. 6, 1852, p. 174.

Rana draytoni Cope

Bull. U.S. Nat.-Mus., No, 34, 1889, p. 441, fig. 114.

TYPE LOCAlity: San Francisco, and the Columbia River.

RANGE: Oregon, California and Lower California. 


\section{Rana grylio SteJNEGER}

Rana grylio Stejneger

Proc. U. S. Nat. Mus., Vol. 24, Oct. 4, 1901, p. 212.

TYPE LOCALITY : Bay St. Louis, Mississippi.

RANGE: Southern Mississippi to peninsular Florida.

\section{Rana onca Cope}

Rana onca Core

in Wheeler's Rep. Expl. W. 100th Mer., Vol. 5, Zoöl. 1875, p. 528, pl. 25 , figs. 1-3.

Rana draytoni onca $\mathrm{COPE}$

Bull. U.S. Nat. Mus., No. 34, 1889, p. 443.

TYPE LOCALITY: Utah.

RANGE: Utah and Nevada.

\section{Rana palustris Le Conte}

PICKEREL-FROG

Rana palustris Le Conte

Ann. Lyc. New York, Vol. 1, Pt. 2, 1825, p. 282.

Rana palustris COPE

Bull. U. S. Nat. Mus., No. 34, 1889, p. 406, fig. 102.

TYPE LOCALITY: Not given.

RANGE: Hudson Bay south to Arkansas and Louisiana and all of the Eastern States.

\section{Rana pipiens SCHREBER}

LEOPARD-FROG

Rana pipicns ScHREBER

Naturforscher, Vol. 18, 1782, p. 185, pl. s.

Rana virescens COPE

Bull. U. S. Nat. Mus., No. 34, 1889, p. 397.

TyPL locality: Pennsylvania.

RANGE: North America, east of the Sierras southward into Mexico.

\section{Rana pretiosa pretiosa (BAIRD and GIRARD)}

Rana pretiosa BAIRD and GIRARD

Proc. Ac. Nat. Sci. Phila., Vol. 6, 1853, p. 378.

Rana tcmporaria pretiosa Cope

Bull. U. S. Nat. Mus., No. 34, 1889, p. 432, fig. 110.

TYPE LOCALITY: Puget Sound, Washington.

RANGE: Utah and Montana west to the Pacific Coast, California

to British Columbia. 


Rana pretiosa luteiventris Thompson

Rana pretiosa luteiventris Thompson

Proc. Biol. Soc. Washington, Vol. 26, March 2\%, 1913, p. 53.

TYPE LoCAlity : Anne Creek, Elko Co., Nevada.

RA NGE: Eureka and Elko Cos., Nevada.

Rana septentrionalis BAtRD

MINK-HROG

Rana septentrionalis BAIRD

Proc. Ac. Nat. Sci. Phila., Vol. 7, 185 t, p. 61.

Rana septentrionalis COPE

Buil. U. S. Nat. Mus., No. 34, 1889, p. 416, fig. 106, pl. 86.

TyPE LOCALITY: Northern Minnesota.

RANGE: Northern New England and northern New York, west through Michigan to Minnesota, Canada northward to Hudson Bay.

\section{Rana sphenocephala (Cope)}

SOUTHERN LEOPARD-FROG

Rana halecina sphenoeephala COPE

Proc. Amer. Philus. Soc., Vol. 23, Dec. 1886, p. $51 \%$.

Rana rireseens sphenocephala COPE

Bull. U. S. Nat. Mus., No. 34, 1889, p. 399, fig. 99.

TYPE LOCALITY: Near St. John's River, Florida. RANGE: Southeastern States

Rana sylvatica Le Conte

WOOD-FROG

Rana sylratica Le Conte

Ann. Lyc. New York, Vol. 1, Pt. 2, 1825, p. 282.

Rana silvatica COPE

Bull. U. S. Nat. Mus., No. 3!, 1889, p. 447, fig. 115.

TYPE LOCALITY: Not stated.

RANGE: Quebec and Nova Scotia to South Carolina westward to

the Great Plains. Southe: outare aut chito.

Rana virgatipes Cope

SPHAGNUM-FROG

Rana virgatipes $\mathrm{COPE}$

Amer. Nat., Vol. 30, 1891, p. 1017.

Ty pe locality: Near Atlantic City, New Jersey.

RANGE: New Jersey to North Carolina. 
BREVICIPITIDAE (FAMILY)

Gastrophryne ( $G E N U S)$

Fitzinger, Syst. Rept., 1843, p. 33

TYPE : rugos $a=$ carolinensis

Gastrophryne areolata (STRECKER)

Engystoma areolata STRECKer

Proc. Biol. Soc. Washington, Vol. 22, June 25, 1909, p. 118.

TYPE LOCALITY: Guadalupe River bottom, Victoria, Victoria

Co., Texas.

RANGE: Southeastern Texas.

Gastrophryne carolinensis (HoLBRooK)

Engystoma carolinense НоцвRоок

N. Amer. Herp., Ed. 1, Vol. 1, 1836, p. 83, pl. 11.

Engystoma carolinense Cope

Bull. U. S. Nat. Mus., No. 34, 1889, p. 385, fig. 97.

TYPE LOCALITY: Charleston, South Carolina.

RANGE: Virginia to Florida, Gulf States to Texas, northward

through Central Valley to southern Indiana.

Gastrophryne texensis (GIRARD)

Engystoma texense Grara

Proc. Ac. Nat. Sci. Phila., 1859, p. 169.

Engystoma carolinense (part), COPE

Bull. U. S. Nat. Mus., No. 34, 1889, p. 385.

TYPE LOCALITY: Rio Seco, Texas.

RANGE: Texas.

\section{Hypopachus ( $G E N U S$ )}

Keferstein, Nachr. Ges. Wiss. Göttingen, July 24, 1867, p. 351 TYPE: seebachii $=$ variolosus

Hypopachus cuneus COPE

Hypopachus cuneus Cope

Proc. U. S. Nat. Mus., Vol. 11, July 5, 1889, p. 395.

Hypopachus cuneus COPE

Bull. U. S. Nat. Mus., No. 34. 1889, p. 388, fig. 98.

type locality: San Diego, Duval Co., Texas.

RANGE: Southern Texas. 




\title{
REPTILIA (CLASS)
}

Laurenti, Syn. Rept., 1768, p. 19

\section{DIAPSIDA (SUBCLASS)}

Osborn, Science (N.S.), Vol. 17, Feb. 13, 1903, p. 276

\author{
LORICATA (ORDER) \\ Merrem, Syst. Amph., 1820, p. 34
}

CROCODYLIDAE (FAMILY)

Crocodylus ( $G E N$ N $T$ )

Laurenti, Syn. Rept., 1768, p. 53

TYPE: niloticus $=$ Lacerta crocodilus Linné in part

\section{Crocodylus acutus Cuvier}

CROCODILE

Crocodilus acutus Cuvier

Ann. Mus. Hist. Nat. Paris, Vol. 10, 180 , p. 55, pl. 1, fig. 3, pl. 2, fig. 5 .

Crocodilus americanus Cope

Ann. Rep. U. S. Nat. Mus. 1898 (1900), p. 173, fig. 2.

TYPE LOCALity : San Domingo.

RANGE: Southern Florida, The Greater Antilles (except Porto

Rico), both coasts of Central America from Mexico to Equador and Columbia.

\section{Alligator ( $G E N C S$ )}

Cunier, Ann. Mus. Hist. Nat. Paris, Vol. 10, 180\%, p. 25

TYPE: lucius $=$ Lacerta alligator Blumenbacis, $1 \% 88$, in part $=$ mississipiensis

\section{Alligator mississipiensis (DAUDIN)}

ALLIGATOR

Crocodilus mississipiensis Daudin

Hist. Nat. Rept., Vol. 2, 1803, p. 412. 
Alligator missisipensis [sic] GRAY

Syn. Rept., 1831. p. 69.

Aligator mississippiensis CoPE

Ann. Rep. U. S. Nat. Mus. 1898 (1900), p. 168, fig. 1.

TYPE LOCALity: "Les bords du Mississippi."

RANGE: Rivers and swamps of the lowlands of the Carolinas,

Georgia and Florida, west to Louisiana, Mississippi and westward to the Rio Grande in Texas.

\title{
SQUAMATA (ORDER)
}

Opper, Ordn. Rept. 1811, p. 14

\section{SAURIA (SUBORDER)}

Macartney, in Ross' Transl. Cuvier's Lect. Comp. Anat., 1802, Vol. 1, table 3

\author{
GEKKONIDAE $(F A M I L Y)$, \\ Phyllodactylus $(G E N U S)$ \\ GraY, Spicileg. Zool., 1830, p. 3 \\ TYPE: pulcher
}

Phyllodactylus tuberculosus WiegmanN

Phyllodactylus tubcroulosus WiEgMANN

Nova Acta Ac. Leop.-Carol., Vol. 17, 1835, p. 241, pl. 18, fig. 2-2a.

Phyllodactylus tuberculosus COPE

Ann. Rep. U. S. Nat. Mus. 1898 (1900), p. 458, fig. 83.

TYPE LOCALITY: "Californien."

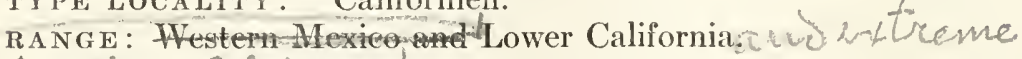
S. wetherm Cabiforich

Phyllodactylus unctus (COPE)

Diplodactylus unctus COPs

Proc. Ac. Nat. Sci. Phila., 1863, p. 102.

Phyllodactylus (Diplodactylus) unctus Bocourt

Miss. Sci. Mexique, Rept., Pt. 2, 1873, p. 43.

Phyllodactylus unctus COPE

Ann. Rep. U. S. Nat. Mus. 1898 (1900), p. 460, fig. 84.

TyPE LOCALITy: Cape St. Lucas, Lower California.

RANGE: Peninsula of Lower California. 
Itanisasty kno (Geres)

ollen, dais, $181 \%$ p. 1163.

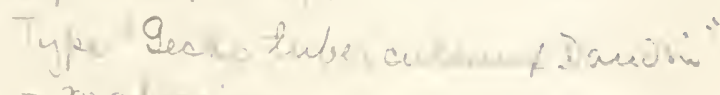
- rmat....ins.

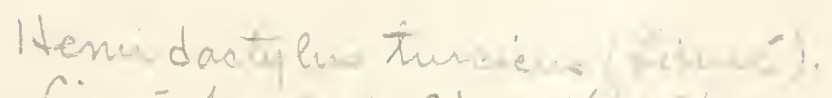

Lime-, Lyar, net, Ed.121166, : $5 t=$

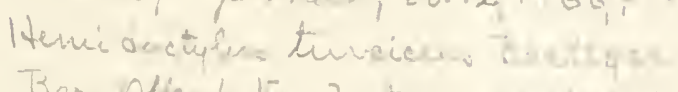

Ter, Ofienl. Ver. hatün, 1:87\% p.57.

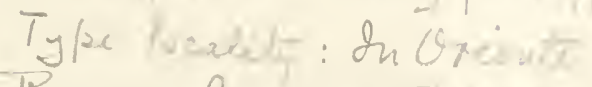

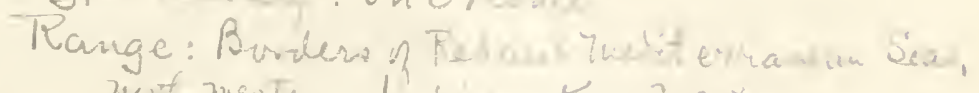
novid matera thioia: Key hear. 
Sphaere dactyhe cineren Wagles.

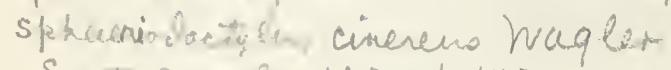
Syzt. ampin. $1830,10.143$.

Ty/u ficaliti : Haiti

Ranye: Haite, Cukn, bla de Prnos, Key trest: 


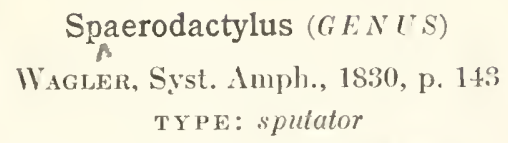

$\checkmark$ Sphaerodactylus notatus BAIRD

Sphaeriodactylus notatus BA1RD

Proc. Ac. Nat. Sci. Phila.. 1858, p. 254.

Sphaerodactylus notatus COPF,

Ann. Rep. U. S. Nat. Mus. 1898 (1900), p. 469 , fig. 85.

TYPE LOCALITY : Key West, Florida.

RANGE: Bahama Islands, Cuba, also southern Florida and the

Keys (perhaps introduced by commerce).

\section{EUBLEPHARIDAE (FAMILY)}

Coleonyx (GENLS)
GraY, Anm. Mag. Nat. Hist., Vol. 16, 1845, p. 162
TYPE: elegans

\section{$\sqrt{ }$ Coleonyx brevis Stejneger}

Coleonyx brevis STEJNEGER

N. Amer. Fauna, No. 7, 1893, p. 163.

Eublepharis variegatus (part) СоРе

Ann. Rep. U. S. Nat. Mus. 1898 (1300), fig. 86.

Ty PE locality: Helotes, Bexar Co., Texas.

RANGE: Texas.

\section{Coleonyx variegatus (BAIRD)}

- Stenodactylus variegatus BAIRD

Proc. Ac. Nat. Sci. Phila., 1858, p. 254.

Coleonyx variegatus COPE

Proc. Ac. Nat. Sci. Phila., 1866, p. 195.

Eublepharis variegatus (part) COPE

Ann. Rep. U. S. Nat. Mus. 1898 (1900). p. $\$ 66$.

TYPE LOCALITY: Rio Grande and Gila Valleys. RANGE: Texas to California, and hertheren Lomer California. 


\section{IGUANIDAE (FAMILY)}

Anolis ( $G E N U S)$

Daudin, Hist. Nat. Rept., Vol. 4, 1803, p. 50

TYPE: bullaris $=$ carolinensis

\section{Anolis carolinensis Vorgt}

"CHAMELEON." ANOLIS

Anolius carolinensis VoIGT

Cuvier's Thierreich, Vol. 2, 1832, p. 71.

Anolis carolinensis COPE

Ann. Rep. U. S. Nat. Mus. 1898 (1900), p. 233, fig. 16.

TYPE LOCALITY: Carolina.

RANGE: North Carolina to Florida, westward through the Gulf region to the Rio Grande.

\section{Ctenosaura ( $G$ EN $U S$ )}

WiegManN, Isis, 1828, p. 371

TYPE: cycluroides

\section{Ctenosaura hemilopha COPE}

Clenosaura hemilopha COPE

Proc. Ac. Nat. Sci. Phila., 1863, p. 105.

Ctenosaura hemilopha COPE

Ann. Rep. U. S. Nat. Mus. 1898 (1900), p. 238, fig. 17.

TyPE LOCALity: Cape St. Lucas, Lower California.

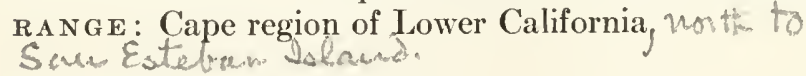

\section{Ctenosaura multispinis CorE}

Ctenosaura multispinis COPE

Proc. Amer. Philos. Soc., Vol. 23, 1885, p. 267.

Ctenosaura multispinis Cope

Ann. Rep. U. S. Nat. Mus. 1898 (1900), p. 240.

TYPE LOCALITY: Dondomúigvillo (sic) = Dondomuiguillo, Oa-

xaca and Batopilas, Chihuahua.

RANGE: Mexico northward to Southern Arizona.

$$
\text { Dipsōsaurus ( } G E N U S \text { ) }
$$

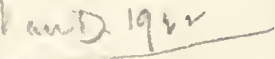

Hallowell, Proc. Ac. Nat. Sci. Phila., Vol. 7, 1854, p. 92

$$
\text { Derva TY PE: dorsalis }
$$

\section{Dipsosaurus dorsalis (BAIRD and Girard)}

Crotaphytus dorsalis BAIRD and GIRARD

Proc. Ac. Nat. Sci. Phila., Vol. 6, 1852, p. 126. 
$\checkmark$ Ctenosaura conspicuosa Diekerson = Lemiloph - K Clenosauna conspienra Dickersan

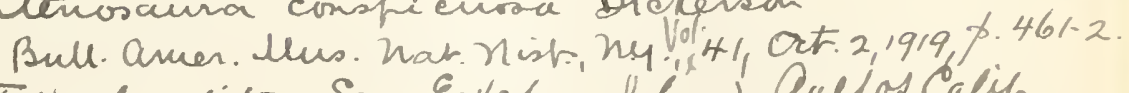
Type lacalitij: San Esteban bland. Quef of Calif. Range: same

$\checkmark$ Ctenosaura insulana Dickeroon = Kemiloph

* Clenosana insulana Dickerson

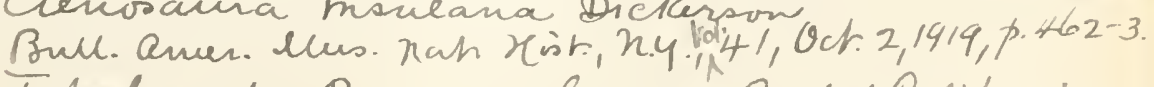
Typelacality: Cerralvo Sland, quef of Califurcia Range: same.

Dipsúsaurus dorsalis hucasenois Van Deuburgh Dipsosaurus dorsalis hucasensis Vauderbugh. Proe. Cal Acad Sci. Ser. 4., Vol. 10, Curq. 6, 1920,

Type localily: San José del Cabo, toren California Range: Cape Relion of Lorres Californera

b.t.o also 
Dipsósaurus carnenensis Vau Deuburgh.

Dipsosourus carmenensis Van Deuburgh.

vce. Pafers Calif. Acad. Sci. rol. 10, hor.23,1922, p. 81

Type locality: Carmen to. Gulf. of Califormia.

Ronge: Carmen und Coronado bls. Forrer Calif.

Dipsosaurus eatalinensis Van Deuburh.

Dipsosaurns catalinersis Van Deubugh.

oce. Pafers Calif. Acad. Sci. Vol.10, hor.23,1927, p. 83.

Type localily: Sautà Catahiua bolaut, Guef of Califormia. Range: Same.

Crotaphytes insularis Vau Deubuash + Slevin.

Crtaphytas insularis vau Dauburgh +5 lEvin.

Proc. Calif. Acas. Sei., Ser. 4, Vof. 9 J July 30, 1921,10.96.

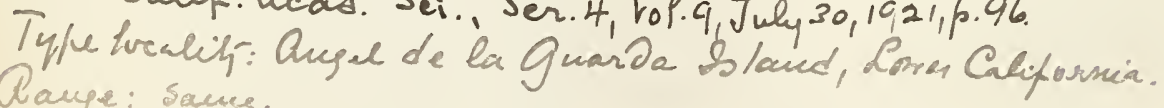

Raupe: Sacuse. 
Dipsosaurus dorsalis Hallowell

Proc. Ac. Nat. Sci. Phila., vol. 7, 1854, p. 92.

Dipsosaurus dorsalis COPE

Ann. Rep. U. S. Nat. Mus. 1898 (1900), p. 243, fig. 18.

TYPE LOCALITY: Colorado Desert, California.

RANGE: From Gape St.-Lueas north along Gulf-Coast of Lower

Galifornia the Colorado and Mojave Deserts, east to the Colorado River.

\section{Crotaphytus (GENUS)}

Holbrook, N. Amer. Herp., Ed. 2, Vol. , 1842, p. 79

TYPE : collaris

Crotaphytus collaris collaris ( $\mathrm{SAY}$ )

COLLARED LIZARD, MOLNTAIN BOOMER

Agama collaris $\mathrm{SAY}_{\mathrm{A}}$

Long's Exp. Rocky Mts., Vol. 2. 1823, p. 252.

Crotaphytus collaris IOLBRook

N. Amer. Herp. Ed. ․ Vol. 2, 1842, p. 79, pl. 10.

Crotaphytus collaris (part) COPE

Ann. Rep. U. S. Nat. Mus. 1898 (1900), p. 248.

TYPE LOCALITY: Verdigris River near its union with the Arkansas River, Oklahoma.

RANGE: Arkansas south to Middle-western and Northwestern

Texas and west to Eastern New Mexico.

\section{Crotaphytus collaris baileyi (STEJNEGER)}

Crotaphytus baileyi STEJNEGER

N. Amer. Fauna, No. 3, 1890, p. 103, pl. 12, fig. 1.

Crotaphytus collaris baileyi STONe

Proc. Ac. Nat. Sci. Phila., 1903, p. 30.

Crotaphytus collaris (part) COPE

Ann. Rep. U. S. Nat. Mus. 1898 (1900), p. 250, fig. 19.

TYPE LOCALITY : Painted Desert, Arizona.

RANGE: Southwestern Texas, southern and western New Mexico,

Arizona, Utah, Nevada, Idaho, southwestern California, northern Mexico, and Lomer Califorsia Eastern

\section{Crotaphytus copeii YaRROW}

Crotaphytus copeii YARROW

Proc. U. S. Nat. Mns., Vol. 5, 1882, p. 441.

Crotaphytus wislizenii (part) COPE

Am. Rep. U. S. Mus. 1898 (1900), p. 255.

TyPe LOCAlity: La Paz, Lower California.

RANGE: Southern Lower California, Cerros and Tiburon Islands. 
Crotaphytus reticulatus BAIRD

Crotaphytus reticulatus BAIRD

Proc. Ac. Nat. Sci. Phila., 1858, p. 253.

Crotaphytus reticulatus COPE

Ann. Rep. U. S. Nat. Nus. 1898 (1900), p. 254, fig. 20.

TyPE LOCALIty: Laredo and Ringgold Barracks, Texas.

RANGE: Extreme southwestern Texas.

Crotaphytus silus STEJNEGER

Crotaphytus silus STEJNEGER

N. Amer. Fauna, No. 3, 1890, p. 105.

Crotaphytus wislizenii (part) COPE

Ann. Rep. U. S. Nat. Mus. 1898 (1900), p. 955.

TYPE LOCALITY: Fresno, California.

RANGE : San Joaquin Valley, California, Washington.

Crotaphytus wislizenii BAIRD and GIRARD

LEOPARD LIZARD

Crotaphytus wislizenii BAIRD and GIRARD

Stansbury's Expl. Surv. Great Salt Lake, 1852, p. 340, pl. 3.

Crotaplytus wislizenii (part) COPE

Ann. Rep. U. S. Nat. Mus. 1898 (1900), p. 255, fig. 21.

ty pe locality: Near Santa Fé, New Mexico.

RANGE: Northern Idaho, Nevada and Utah, southward into

Mexico, Lower and southern California.

\section{Sauromalus ( $G E N U S)$}

Duméril, Arch. Mus. Hist. Nat. Paris, Vol. 8, 1856, p. 535

TYPE : ater

- Sauromalus ater DumériL

CHUCK-WALLA

Sauromalus ater DunéniL

Arch. Mus. Hist. Nat. Paris, Vol., 8, 1856, p. 536, pl. 23, fig. 3.

Sauronnalus ater COPE

Ann. Rep. U. S. Nat. Mus. 1898 (1900), p. 266, fig. 23.

TYPE LOCALITY: Unknown.

RANGE : Southern -Nevada, southwestern Utah, Arizona, south-

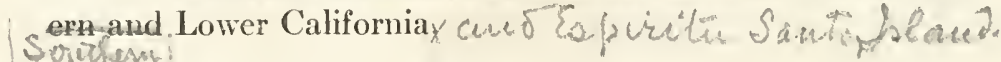

Sauromalus hispidus STEJNEgER

Sauromalus hispidus Stejneger

Proc. U. S. Nat. Mus.. Vol. 14, Oct. 27, 1891, p. 409.

Sauromalus hispidus Cope

Ann. Rep. U. S. Nat. Mus. 1898 (1900), p. 264, fig. 22. 
Crotabiytus diciterar.ae Achmidt Crotaphy tus dickerarnas Schmidt

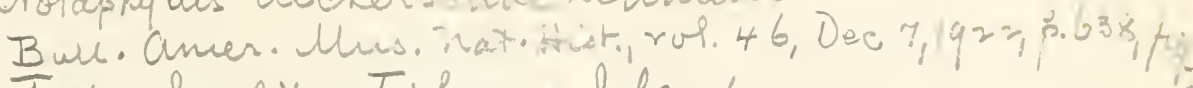
Type locality: Tiburon dalaud. Rame: save.

Sauromahns obesus (Baird) Euphryne obtesa Bairo thos. acas. hot. Sci. Philos, $1858, p, 253$. Sannomalna obesus schmidt Bull. Amer uns. hat. Atior, vol, +6, Dec. 7, 19rr,p.641. Type locality: Fort yuma, arizona.

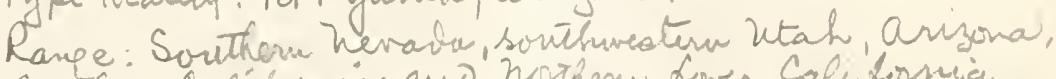

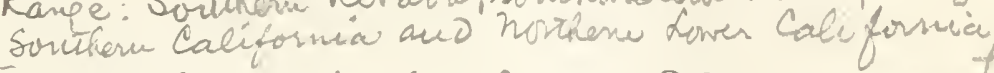

Sauromalus interbrachialis Dickersow = ate? Saunomalus interbrachialis Dickerson

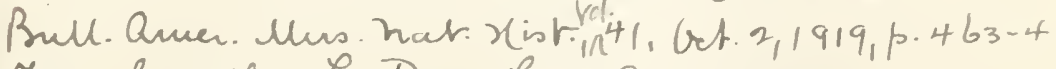

Iype localily: La Pay, Lorrer California

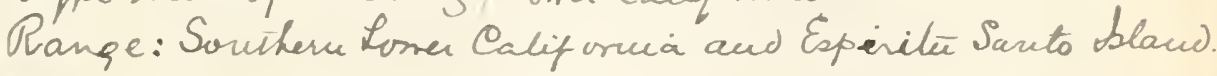

Sauromalus tonnsendi Dickerson Sauromalus townsenor Dickesson

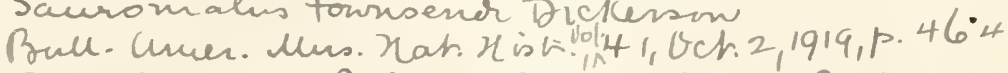

Type lucality: Jiburon Drlans, quef of Califoncia

Range: Ii inson bland und fossiby abjacent corats region of Sonora, on sxico.

Sauromalno rarius Dickersom

Sauromalus rarius Hickerson

Bull. Aver, Mus. hat. X ist. 14 1, Oet, 2,1919, p. 464-5

Typelucalily: San Esteban oland, fuef of aliforcia.

Rave: Sасие.

$\checkmark$ Sauromalus Slevini Tan Denbugh.

Sauromalus Slevini Van Deuburgh.

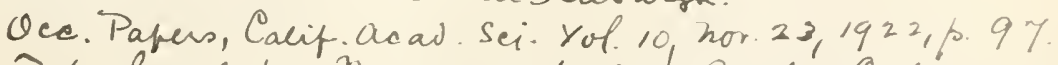

Type locality: Thonserrate sel. Quefof Califormia.

Raupe: Monserrate, Carmen aud Connalo 2.

Lona calif. 
I Callisaurus carmenensin Dickersm = gabloi

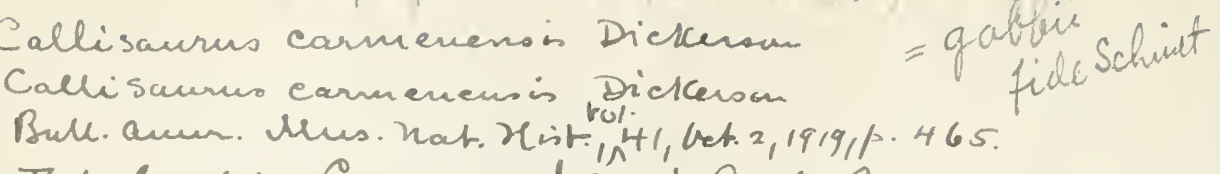

Tytuelvality: Carmen heacid, Grefor Califurnion. Range: sacue.

vantralis

-allisaurus imusitatus Dickerson

$\left(\begin{array}{c}\text { subst find } \\ \text { Sohniet }\end{array}\right)$

Callisaurns imusitatus Dickerson

Bull. Amer. Mus. Hat. Nist., Vo1. 41, vet. 1919, p. $4605=6$.

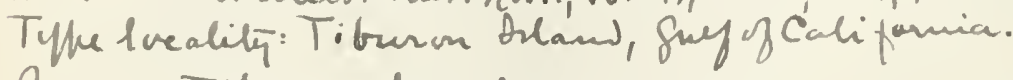

Raupe: Tiburon thloul and region aberst graymas maxico.

Callisanns plasticus Dickerson = gabthi fille schiett Callisanns Hlasticus Dickerson

Bulle. Ancen. Uns. hat: Hist, Vol. 41, Oct. 2,1919, p. 466.

Typre lealits; Aqua Verde Bay, furrer Califurmia. Range: Centrue zartern curast region, Lover Califarmia and San José Drand.

$\checkmark$ Callisanns splendidus Dickerson. Callisamms splentides Dickersm Bull. Amer. Mhr. Wat, Xist, Vo1. 41, Och. 2, 1919, p. 467.

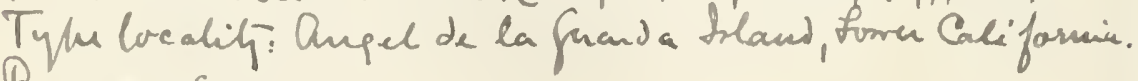
Range: Same.

Callisaurus ventrakis gabbii Cofe Calle srem. ventracis quatio Cote

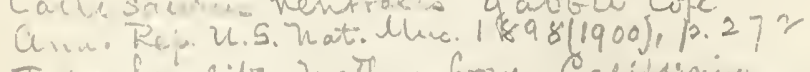
Type focalitï: hothene hower Califfomise. Raye. Lower Calitomin, decerts of Sontitem Colifornio and adjacent deeents ni Arigonos.

lima

unia (genus:).

to frelos Baird, Proc Ocai. har. Sci. Phila, 1858, p, 253. Callisousons Tylu: notalá

hitt one opeciess. 
TYPE LOCAlity: Angel de la Guardia Island, Gulf of California. RANGE: Same as type locality.

\section{Callisaurus $(G E N T S)$}

Blaintille, Nouy, Ann. Mus. Hist. Nat. Paris, Vol. 4, 189.5, p. 986 TYPE: draconoides

$\sqrt{ }$ Callisaurus crinitus Cope

Callisaurus crinitus: $\mathrm{COPE}$

Amer. Nat., Vol. 30, Dec. 5, 1596. p. 1049.

TYPE LOCALITY: Ballenas Bay, Lower California.

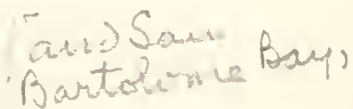

RANGE: Ballenas Bay, Lower California.

Callisaurus draconoides Blainville

Callisaurus draconoides B baINville

Nouv. Ann. Mus. Hist. Nat. Paris, Vol. ‘, 1835. p. 286, pl. 24, fig. ‥

Callisaurus draconoides COPE

Ann. Rep. U. S. Nat. Nus. 1898 (1900), p. 269, fig. 94.

TYPE LOCALITY: California.

RANGE: Southern Lower California.

Callisaurus ventralis ventralis (HALLOWELL)

GRIDIRGN-TAILED LIZARD

IIomalosaurus ventralis HALLOWELL

Proc. Ac. Nat. Sci. Phila., Vol. 6, 1859, p. 179.

Callisaurus ventralis BAIRD

U. S. Mex. Bound. Surv.. Vol. 1, Pt. ․, 1859, Rept., p. 8.

Callisaurus draconoides rentralis COPE

Amn. Rep. C. S. Nat. Mis. 1898 (1900). p. 979, fig. 25.

TYPE LOCAlity: New Mexico west of Rio Grande.

RANGE: Texas to California, north to Nevada, southward into

Mexico at least to Guaymas.

Callisaurus ventralis myurus RICHARDsoN

Callisaurus ventralis myurus RichARDSON

Proc. C.S. Nat. Mius., Vol. 48, Jan. 19, 1915. p. 408.

ty

RAxge: Region about Pyramid Lake, Nevada.

thise

Callisaurus notatus (BAIRD)

Uma notata BAIRD

Proc. Ac. Nat. Sci. Phila., 1858, p. 253.

Gallisaurus notatus COPE

Amer. Nat. Vot $30,-1896, p,-1049$.

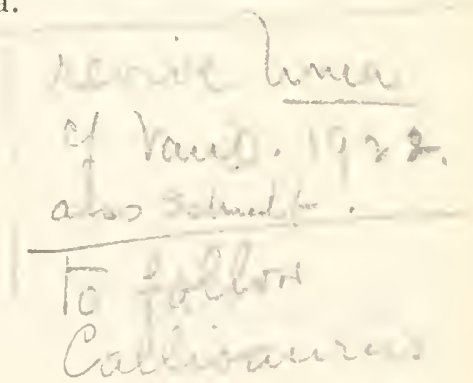


Uma notata Cope

Ann. Rep. U. S. Nat. Mus. 1898 (1900), p. 27\%, fig. 27.

TYPE LOCALITy : Mojave Desert, California.

RANGE: Yuma and Mojave Deserts.

Callisaurus scoparius (Cope) ${ }^{1}$

Uma scoparia Cope

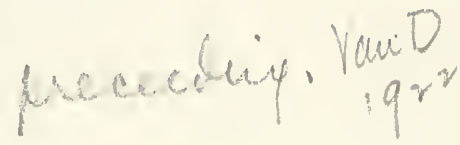

Amer. Nat., Vol. 28, 1894, p. 435, figs. 3-4.

Uma scoparia Cope

Ann. Rep. U. S. Nat. Mus., 1898, (1900), p. 282, fig. 29.

TYPE LocAlity: Tueson, Arizona.

RANGE: Arizona Deserts.

\section{Hoibrookia ( $G E N U S)$}

Grrard, Proc. Amer. Assoc. Adv. Sci., New Haven, Vol. 4, 1851, p. 201 TYPE: maculata

Holbrookia maculata maculata (GIRARD)

Holbrookia maculata GIRARD

Proc. Amer. Assoc. Adv. Sci., New Haven, Vol. 4, 1851, p. 201.

Holbrookia maculata maculata CoPE

Ann. Rept. U. S. Nat. Mus. 1898 (1900), p. 293, fig. 33.

T Y PE LOCA Lit Y : Opposite Grand Island, Platte River, Colorado. RANGE: Northern Texas west to Arizona and northward to Wyoming and Nebraska.

\section{Holbrookia maculata approximans (BAIRD)}

IIolbrookia approximans BA1RD

Proc. Ac. Nat. Sci. Phila., 1858, p. 253.

Ilollroolia maculata approximans Stejneger

N. Amer. Fauna, No. 3, 1890, p. 109.

Holbrookia maculata approximans Cope

Ann. Rep. U. S. Nat. Mus. 1898 (1900), p. 297.

TyPE LOCALITY: “Lower Rio Grande."

RANGE: Southern New Mexico and Arizona, south into northern

Mexico.

\section{Holbrookia maculata flavilenta Cope}

IIolbroolvia maculata flavilenta CopE

Proc. Ac. Nat. Sci. Phila., 1883, p. 10.

IIolbroolia maculata flavilenta CoPE

Ann. Rep. U. S. Nat. Mus. 1898 (1900), p. 298 , fig. 34.

1 In the original description Cope says "I name the specimen from Tucson Uma scopifera" but a few lines latcr calls it Uma scoparia and does so in all later publications. 
sut

tolbratia pueahra Achmult tolrookix prechua Schmidt aneer. Inus. hor., ho. 22, Dea.1,1921,p.1.

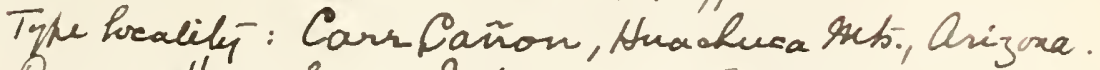
Range: Huachuca kth., East of kogoles, Arizona.

Nolbrobice onaculatā caupi Schmict. - a'. Stet Hehrookei enaculata caupi dchmilt. aneur. Hus. Hor, Ho.22, Dec. 1, 1921,p.1. Type Prealit5: $8 \mathrm{~m}$. . . L. of Odamawa, apade C., Arizon. Aange: Sontheru paut of the Colorado Hatean in ho. arizona, possibly E. nito new mesoico tot not $\pi$. to Wtah.

Hallvokia dickersonae Sahuidt. Hallrooki dicheromae Schuidt. Ama. Mus. Nor., ho.22, dec.1,1921,p.2.

Sype bralif5: Castanuelas, Coahila, hes. Rampe: Kuorn mh trom the thre loculit-acis alaneos de Purras, Coahuila, Mesico. 
$\checkmark$

Hobbrarcia elegans Rocourt

Hobroracia elegans Bocowrt

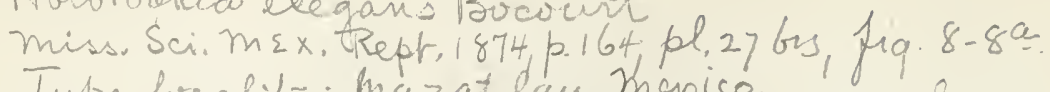

Type lvaality: Inazat lau Moxico.

Range: Trestern mexico from simaloa northward Io Juscon, arizura. 
TYPE LOCALitY: Lake Valley, southern New Mexico.

RANGE: Southern New Mexico, southern and western Arizona.

Holbrookia maculata lacerata $\left(\mathrm{COP}^{\mathrm{N}} \mathrm{E}\right)$

IIolbrookia lacerata COPE

Bull. U.S. Nat. Mus., No. 17, 1880, p. 15.

Holbrookia maculata lacerata STEJNEGER

N. Amer. Fauna, No. 3. 1890, p. 109.

IIolbrookia maculata laccrata COPE

Ann. Rep. U. S. Nat. Mus. 1898 (1900), p. 292, fig. 32.

TYPE LOCALITY: Erath Co.; west of the Upper Brazos, Comanche Co.; on the Guadalupe River in Kendal or Comal Co. RANGE: Texas, middle district east of the plains and west of the timbered region, from the northern boundary south to the Rio Grande.

Holbrookia propinqua BaIRd and Girard

IIolbrookia propinqua BAIRD and GiRARD

Proc. Ac. Nat. Sci. Phila., Vol. 6, 18.52, p. 126.

Holbrookia propinqua COPE

Ann. Rep. U. S. Nat. Mus. 1898 (1900), p. 289, fig. 31.

TYPE LOCALITY: Between Indianola and San Antonio, Texas. RANGE: Texas.

\section{Holbrookia texana (Troschel)}

Cophosaurus texanus Troschel

Arch. Naturg. Vol. 1, 1850 (1852), p. 389, pl. 6.

IIolbrookia texana BAIRD and GIRARD

Proc. Ac. Nat. Sci. Phila., Vol. 6, 1852, p. 124.

IIolbrookia texana COPE

Ann. Rep. U. S. Nat. Mus. 1898 (1900), p. 986, fig. 30.

TYPE LOCALITY: The German colony of Neubraunfels, on the

Guadalupe River in Western Texas, Lat. $28^{\circ} \mathrm{N}$.

RANGE: Texas and eastern New Mexico.

Uta $(G E N U S)$

Baird and Girard. Stansbury's Expl. Surv. Vall. Great Salt Lake, 1852 , p. 345

Uta auriculata Cope

TY PE: stansburiana

Uta auriculata COPE

Proc. Boston Soc. Nat. Hist.. Vol. 14, 18 1, p. 303.

TyPE LOCALITY: Socorro Island, Revilla Gigedo Archipelago, Mexico.

RANGE: The type locality. 
Uta clarionensis TOWNSEND

Uta clarionensis 'TownSEND

Proc. U. S. Nat. Mus., Vol. 13, Sept. 9, 1890, p. 143.

ty Mexico.

RANGE: Same as type locality.

Uta graciosa (HALLOWELL)

Urosaurus graciosus HALlowell

Proc. Ac. Nat. Sci. Phila., Vol. 7, 1854, p. 92.

Uta graciosa BAIRD

U. S. Mexican Bound. Surv., Vol. 2, Pt. 2, 1859, Rept., p. 7.

Uta graciosa COPE

Ann. Rep. U. S. Nat. Mus. 1898 (1900), p. 325, fig. 46.

TYPE LOCALITY: "Lower California" - with the intention of saying southern California.

RANGE: Southern California Arizona. Nevadaculd wortheraterse

Uta levis Stejneger forven Caldeformia.

Uta levis STEJNEGer

N. Amer. Fauna, No. 3, 1890, p. 108.

Uta levis Cope

Ann. Rep. U. S. Nat. Mus. 1898 (1900), p. 313, fig. 40.

type locality: Tierra Amarilla, New Mexico.

RANGE: New Mexico.

Uta martinensis $V_{\text {AN }}$ DeNBURGH

Uta martinensis $\mathrm{V}_{\mathrm{AN}}$ Denburgh

Proc. California Ac. Sci., Ser. 3, Vol. 4, 1905, p. 18, pl. 6.

TYPE LOCALITY : San Martin Island, Lower California.

RANGE: San Martin Island.

Uta mearnsi STEJNEGER

Uta mcarnsi STEJNEGER

Proc. U. S. Nat. Mus., Vol. 17, Nov. 30, 1894, p. 589.

Uta mearnsii Cope

Ann. Rep. U. S. Nat. Mus. 1898 (1900), p. 204, fig. 37.

TYPE LOCAlity: Summit of the Coast Range, Mexican Boundary of California.

RANGE: Boundary region of southern and Lower California.

\section{Uta microscutata VAN Denburgh}

Uta microscutata VAN DENBURGH

Proc. California Ac. Sci., Ser. 2, Vol. 4, 1894, p. 998. 
- iltá concinna Dickerson M L L E ulà concinna Dickerson. valis füle Sehuet

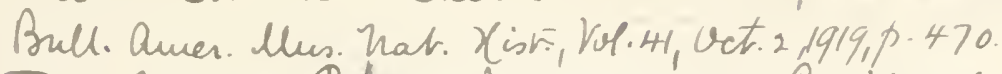
Type localif: Cedros beand, fuef of Californéa. Rampe: Cedrosand Latividad Islaus, Lomer cacifomia

I Wà mannoptorus Dieklesson Ulà mannophoreso biekeroon.

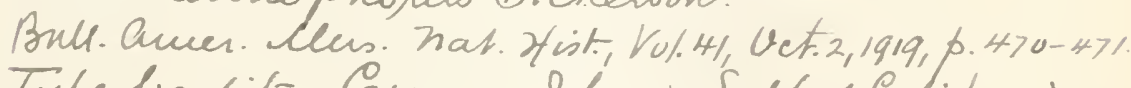
Tyle brealits: Carmen hlawd, Sulf of Paliforina. Ramp: sacue.

Wà barra Dickuron $=U$. s. Myares 1 ?

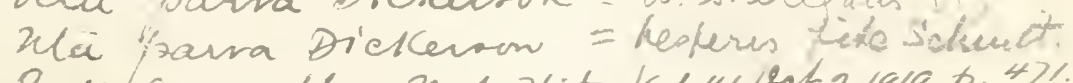

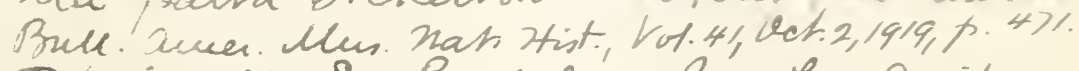

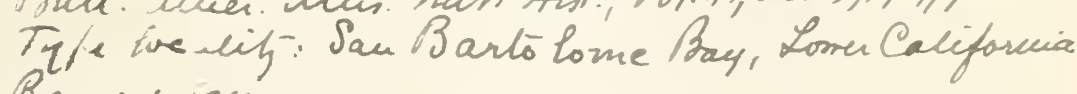
Raye: sacu.

$\checkmark$ UL squamata Dickusm. Wia squacuata bickerson

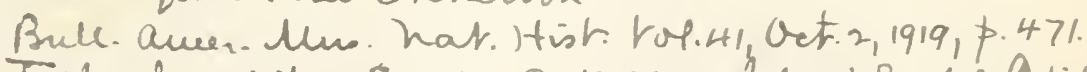
The 1reulity: Sauta Catralina hland, fuef of Lalifaricia Raves: save.

Wà wrighte Shmidt. $=$ livis fie taud Wta wriqhti Schuidet anver. mus. hor., ho.22, Dec.111921,p.3

Tyhs lveality: Granidyulch, Sau luauco., Vtat.

Pange: hresteru Colorab and eontreasteru and sontheru hiak.

Iste yajuri tchmidt nta gavori Achmidt. Muer. Mus. hor., ho.22, Dea.1, 19-1,3.3. Tyhe lrealit: Calradia, fallico, inesico Rave: Pulisco and Michoacas 'mu ico 
Tita tubuculata Sehmidt nta tiberculata Schriedt Amen. Mus. Nor., ho. 22, Dec.1, 1921, p.4 Type bocalifs: Colinea, Colina, huxico. of Ranpe: Colina ans folisco, Puxico.

\& Uta nelsoni Sehmidt hà nelsoni schmidt.

Amer. Hus. Hor., ho. 22, wae.1, 1921,s.

Tylue loralif-: Cuicat lan, Daxaca, Mupico. Rampe: The tothe lvealif.

Wtà nolas cencis rau Jeubuyh o Slevin. nià nolascensis vandeutumh + Alemin. Prre. Calif. Acas. Sci, Ser. 4, tol.11, Dec.17,1921, p.395 Type hralit5: SauPebro holas co blant, Oonen Califorma. Racye: same.

Uta stans-buriana stejneger Schuidt Wta etansburiana etejnegeri Schuidt. Amer. Mns. hov. ho.15,1941, 1 . Type Proality: Mount of Ory Cañon, alanogordog otero County,

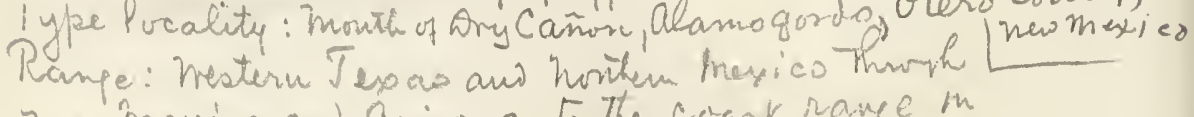
new mesico aud Arizona to The coast rasye in Califorma; nurderastern homer Californa:; anyel de la Guarda blans 
Uta parviscutata COPE

Ann. Rep. U. S. Nat. Mus. 1898 (1900), p. 324, fig. 4.5.

TYPe Locality: San Pedro Martir Mountains, Lower California. In whtheres formen Catiomia

RANGe: San Pedro Martir Mountain and San José Island, Lower California.

\section{Uta nigricauda CoPE}

Uta nigricanda Cope

Proc. Ac. Nat. Sci. Phila., 186it, p. 176.

Uta nigricauda COPE

Ann. Rep. U. S. Nat. Mus, 1898 (1900), p. 329, fig. 44.

TYPE LOCALITY: Cape St. Lucas, Lower California.

RANGE: Southern Lower California.

Uta ornata BArrd and Grrard

Uia ornata BAIRD and GiRARD

Proc. Ac. Nat. Sci. Phila., Voi. 6, 1852, p. 126.

Uta ornata Cope

Ann. Rep. U.S. Nat. Mus. 1898 (1900), p. 315, fig. 41.

ty lo locality : Rio San Pedro, Texas, and Sonora.

RANGE: Utah, Nevada, western Texas west to southern Cali-

fornia, northern Mexico.

Uta palmeri STEJNEGER

Uta palmeri STEJNEGER

N. Amer. Fauna, No. 3, 1890, p. 106.

Uta palmerii Cope

Ann. Rep. U. S. Nat. Mus. 1898 (1900), p. 313, fig. 39.

TYPE LOCALITY: San Pedro Martir Island, Gulf of California.

RANGE: San Pedro Martir Island, Gulf of California.

\section{Uta repens Van Denburgir}

Uta repens Van Denburgil

Proc. California Ac. Sci., Ser. 2 , Vol. 5, 1895, p. 102, pl. 7-8, figs. a-e.

Uta repens $\mathrm{COPE}$

Ann. Rep. U. S. Nat. Mus. 1898 (1900), p. 303, fig. 36.

TyPe locality: Comondu, Lower California.

RANGE : Lower California.

Uta stansburiana stansburiana (BAIrD and Girard)

Uta stansburiana BAIRD and Girard

Stansbury's Expl. Surv. Vall. Great Salt Lake, 1852, p. 345, pl. 5, figs. 4-6.

Uta stansburiana COPE

Ann. Rep. U. S. Nat. Mus. 1898 (1900), p. 306, fig. 38. 
TYPE LOCALITY: Salt Lake Valley.

RANGE: Parts of Utah, Idaho, Nevada and southern California.

- Uta atansburiana elegans (YARRow)

Uta elegans Yarrow

Proc. U. S. Nat. Mus., Vol. 5, Sept. 29, 1882, p. 442.

Uta stansburiana elegans Ricinardson

Proc. U. S. Nat. Mus., Vol. 48, Jan. 19, 1915, p. 413.

Ty PE Locality: La Paz, Lower California.

RA NGE: Southern and Lower California, Arizona,-New-Mexico,

Texas, and parts of northern Mexico (in deserts).

$\checkmark$ Uta stansburiana hesperis RICHARDSON

Uta stansburiana hesperis RICHARDSON

Proc. U. S. Nat. Mus., Vol. 48, Jan. 19, 1915, p. 415.

type locality: Arroyo Seco Cañon, near Pasadena, Los

Angeles County, California.

RANGE : Coast region of southern California.

\section{Uta stellata Van Denburgh}

\section{Uta stellata $\mathrm{V}_{\mathrm{AN}}$ DenBURGH}

Proc. California Ac. Sci., Ser. 3. Vol. 4, 1905, p. 21, pl. 8.

TYPE LOCALITY: San Benito Island, Lower California.

RANGE: The type locality.

\section{vernato.}

Uta symmetrica BAIRD

Uta symmetrica BAIRD

Proc. Ac. Nat. Sci. Phila., 1858, p. 253.

Uta symmetrica Cope

Ann. Rep. U.S. Nat. Mus. 1898 (1900), p. 317, fig. 42.

type locality : Fort Yuma, Arizona.

RA NGE: Southern Arizona, Southeastern-California.

\section{Uta thalassina Cope}

Uta thalassina CoPE

Proc. Ac. Nat. Sci. Phila., 1863, p. 104.

Uta thalassina COPE

Ann. Rep. U. S. Nat. Mus. 1898 (1900), p. 301, fig. 35.

TYPE LOCALITy: Cape St. Lucas, Lower California.

RANGE: Southern Lower California.

\section{Coformedo deand and Colerabo Rica Vallu.}


$\checkmark$ Wta slevini Van Denburgh uta relevini Vau Denburgit.

occ. Popers Calif. Acad. Sci. Vol, 10, Nor. $23,1922,5.94$

iyte lucality: Minjia Dland. Yueff. of Califonia

Range: Angel de la Guarda Lze aud he jia Lal. Lon a Calif.

? To a w in oru.

$+c p .207$.

Sator (genus) (to follow retas).

Dicterson, Bule. Amer. Mus. Nat. Xist, Hot.41, oct $2,1919, p .468-9$.

Type: grandaevers.

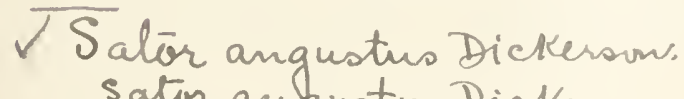

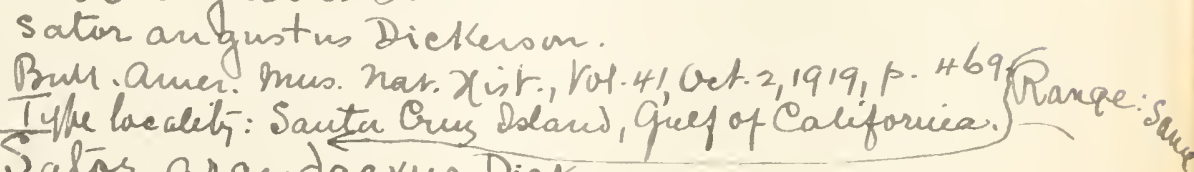

Satar grandaevns dickerson.

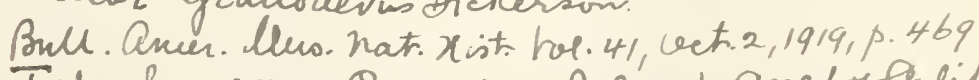

Tilhe localit: Cerrulvo Doland, guef of Califormia.

Range: Sacue. 



\section{Sc eloporus ( $G E N U S)$}

Wiegmann, Isis, 1828, p. 369

TYPE: torquatus

$\checkmark$ Sceloporus clarkii BAIRD and Girard

Sceloporus clarkii BaIRD and GIRARD

Proc. Ac. Nat. Sci. Phila., Vol. 6, 1852, p. 127.

Sceloporus clarkii Cope

Ann. Rep. U. S. Nat. Mus. 1898 (1900), p. 358, fig. 54.

TYPE LOCALITY: Sonora.

RANGE: New Mexico, Arizona, and Northern Mexico.

Sceloporus consobrinus consobrinus (BAIRD and Girard)

Sceloporus consobrinus BAIRD and GIRARD

Marcy’s Expl. Red River, 1853, Rept., p. 237, Zoöl., pl. 10, figs. 5-12. Sceloporus undulatus consobrinus Cope

Ann. Rep. U. S. Nat. Mus. 1898 (1900), p. 377, fig. 60.

TyPE locality: Oklahoma, Roger Mills Co., Red River.

RANGE: The Dakotas, southward to Texas and westward to

California.

\section{Sceloporus consobrinus garmani (BoulENGER)}

Sceloporus garmani BouleNGer

Proc. Zool. Soc. London, 1882, p. 761, pl. 56.

type Lochlity: Near Pine Ridge, South Dakota.

RANGE: Region about type locality.

Sceloporus couchii BAIRD

Sceloporus couchii BAIRD

Proc. Ac. Nat. Sci. Plila., 1858, p. 254.

Sceloporus couchii COPE

Ann. Rep. U. S. Nat. Mus. 1898 (1900), p. 395, fig. 66.

Lysoptychus lateralis $\mathrm{COPE}$

Aun. Rep. U. S. Nat. Mus. 1898 (1900), p. 329, fig. 47.

Ty PE locality: Santa Caterina, Nuevo Leon, Mexico.

RANGE: Northern Mexico to southern Texas.

\section{$\checkmark$ Sceloporus disparilis Stejneger}

Sceloporus disparilis STEJNEGER

Proc. Biol. Soc. Washington, Vol. 29, Dec. 16, 1916, p. 228.

TyPE LOCALITY: Lomita Ranch, six miles north of Hidalgo,

Texas.

RANGE: Extreme southern corner of Texas and northeastern Mexico. 
Sceloporus elongatus STEJNEGER

Sceloporus elongatus STEJNEGER

N. Amer. Fauna, No. 3, 1890, p. 111.

Sceloporus undulatus (part) COPE

Anu. Rep. U. S. Nat. Mus. 1898 (1900), p. 368.

ty pe locality: Moa Ave., Painted Desert, Arizona.

RANGE: The Painted Desert region.

Sceloporus graciosus graciosus (BAIRd and GrRARD)

Sceloporus graciosus BaIro and Grrard

Stansbury, Expl. Surv. Vall. Great Salt Lake, 1852, p. 346, pl. 5, figs. 1-3.

Sceloporus graciosus graciosus: CAMP

Univ. California Publ. Zoöl., Vol. 17, Dec. 28, 1916, p. 67.

Sceloporus graciosus Cope

Ann. Rep. U. S. Nat. Mus. 1898 (1900), p. 386, fig. 63.

TYPE LOCALITY: Valley of Great Salt Lake.

RANGE: Oklahoma to California and Utah, Nevada and-Oregon.

Sceloporus graciosus vandenburgianus (COPF)

Sceloporus randenburgianus COPE

Amer. Nat., Vol. 30, 1896, p. 834.

Sceloporus graciosus vandenburgianus GRINNELL and CAMP

Univ. California Publ. Zoöl., Vol. 17, July 11, 1917, p. 159.

Sceloporus vandenburgiamus COPE

Ann. Rep. U. S. Nat. Mus. 1898 (1900), p. 390, fig. 64.

type locality: Summit of the Coast Range, San Diego Co.

California.

RANGE: Mountain ranges south of Ventura Co. California, into

untherm Rower Caluformia.

Sceloporus jarrovii Cope

Sceloporus jarrovii COPE

Wheeler's Rep. Surv. W. 100th Merid., Vol. 5, 1875, p. 569, pl. 23. fig. 2-2c.

Sceloporus jarrovii COPE

Ann. Rep. U. S. Nat. Mus. 1898 (1900), p. 345, fig. 49.

TYPE LOCALITY: Southern Arizona.

RANGE: Arizona.

Sceloporus licki Van Denburgh

Sceloporus licki Van Denburgh

Proc. California Acad. Sci., Ser. 2, Vol. 5, 1895, p. 110, pl. 10.

Sceloporus lickii COPE

Ann. Rep. U. S. Nat. Mus. 1898 (1900), p. 363.

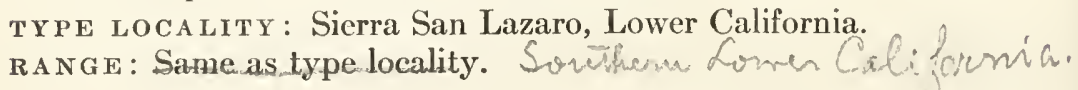


Sedoporers hireatulus Diekerson.

Sceloporers lineatulus diekenson

Bull. Amer. llus. hat X x st. Vul. 41, Get. 2, 1919, f. 467

Typhe Realit: Santa Calalina sland, Qulf Bf Califormia.

Tanpe: sacuce.

Sceloporis monserratensis rau Deubugh o Slenin. suloperns monserratensis tan Dentugh o Slerin.

Proc. Calif. Aead. Sci, Ser. 4, Vol. "1, Dec.17,1921,p.396.

Tyle lrealib-: Monserrate Dsland, forres Califarnia.

Range: same.

Sceloporus graciosus gracilis (Pairo a Tiraw)

Sceloporua graceles Bairs \& Geraid

Prec acad. Lat. Sci. Phila., rol. 6, 1852, p. 175

Socoporno gracions gracilis $\operatorname{ran} D$ enburgh.

oce. Paters Calif Acad. Sci. Fol 10, hor. 23,1922,19 2 so Trke lerality: Oreqon.

Range: trestern Orejon and hasieiter + Mr. Calif. 

Sceloporus magister HaLlowelL

Sceloporus magister HaLlowell

Proc. Ac. Nat. Sei. Phila., 185̃, p. 93.

Sceloporus clarkii (part) CopE

Ann. Rep. U. S. Nat. Mus. 1898 (1900), p. 358.

TYPE LOCALITY: Yuma, Arizona.

RANGE: Southeastern California, western Arizona, southern Nevada, southwestern Utah, no.thwesterm Lomer inabigorinica

Sceloporus merriami STEJNEGER

Sceloporus merriami STEJNEGER

Proc. Biol. Soc. Washington, Vol. 17, Feb. 5, 1904, p. 17.

TYPE LOCALITY: East Painted Cave near moutl of Pecos River,

Rio Grande Valley, Texas.

RAnge: Rio Grande Cañon, Western Texas.

Sceloporus occidentalis occidentalis (BAIRD and GIRARD)

Sceloporus occidcntalis BAIRD and GiRARD

Proc. Ac. Nat. Sci. Phila., Vol. 6, 1852, p. 175.

Sceloporus occidentalis occidentalis CAnP

Univ. California Publ. Zoöl., Vol. 17. Dec. 28, 1916, p. 65.

TYPE LOCALITY: Benicia, California.

RANGE: Northern and nortl central California.

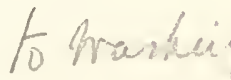

Sceloporus occidentalis beclsi (VAN DENBURGH)

Sceloporus becki TAx Dexburgh

Proc. California Ac. Sci., Ser. 3, Zoöl., 4, June 15, 1905, p. 9, pl. 4.

Sceloporus occidentalis bechi GRINAELL and CAMP

Univ. of Calif. Publ. Zoöl., Vol. 17, July 11, 1917, p. 162.

TyPe LocAlity: San Miguel Island, Santa Barbara Co., Cali-

fornia.

RANGE: Islands off coast of Santa Barbara County, California.

\section{Sceloporus cccidentalis bi-seriatus (HALlowell)}

Sceloporus bi-seriatus HALLOWELL

Proc. Ac. Nat. Sci. Phila., Vol. 7, 1854, p. 93.

Sceloporus occidentalis bi-seriatus CAMP

Univ. California Publ. Zoöl., Vol. 17, Dec. 28, 1916, p. 65.

Sceloporus biseriatus Cope

Ann. Rep. U.S. Nat. Mus. 1898 (1900), p. 381, fig. 61.

Ty PE Locality: El Paso Creek, Tejon Valley, Texas.

RANGE: Texas westward to California, also Utah, Idaho and

Nevada. 
Sceloporus occidentalis taylori CAMP

Sceloporus occidentalis taylori CAMP

Univ. California Publ. Zoöl., Vol. 17, Dec. 28, 1916, p. 65.

TYPE LOCALITy: Halfway between Merced Lake and Sunrise

Trail (Ecko Creek Basin), altitude 7500 feet, Yosemite National Park, California.

RANGE: Yosemite National Park, above 7000 feet altitude.

\section{Sceloporus orcutti STEJNEGER}

Sceloporus orcutti STEJNEGER

N. Amer. Fauna. No. 7, May 31, 1893, p. 181, pl. 1, figs. 4a-c.

Sceloporus orcutiii COPE

Ann. Rep. U. S. Nat. Mus. 1898 (1900), p. 354, fig. 52.

ty pe locality: Milquatay Valley, San Diego County, Cali-

fornia.

RANGE : Southern California.

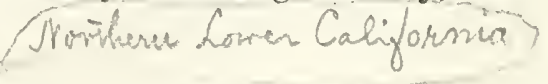

Sceloporus scalaris WeigmanN.

Sceloporus scalaris WiegmanN

Isis, 1828, p. 370.

Sceloporus scalaris Cope

Ann. Rep. U. S. Nat. Mus. 1898 (1900), p. 401, fig. 68.

TYPE LOCALITY: Mexico.

RANGE: Mexico northward to the U.S. boundary.

Sceloporus spinosus floridanus (BaIkD)

Sceloporus floridanus BAIRD

Proc. Ac. Nat. Sci. Phila., 1858, p. 254.

Sceloporus spinosus floridanus STEJNEGER

N. Amer. Fauna, No. 7, May 31, 1893, p. 181.

Sceloporus spinosus (part) COPE

Ann. Rep. U. S. Nat. Mus. 1898 (1900), p. 364, fig. 55.

type locality : Pensacola, Florida.

RANGE: Extreme western Florida to Texas, New Mexico and northern Mexico.

\section{Sceloporus torquatus poinsettii (BAIRD and GIRARD)}

Sceloporus poinsettii BAIRd and GiRARD

Proc. Ac. Nat. Sci. Phila., Vol. 6, 1852, p. 126.

Sceloporus torquatus poinsettii CopE

Proc. Amer. Philos. Soc., Vol. 29, 1885, p. 402.

Sceloporus torquatus poinscttii COPE

Ann. Rep. U. S. Nat. Mus. 1898 (1900), p. 350, fig. 51.

ty pe Locality: Rio San Pedro, Texas, and Sonora, Mexico. RANGE: Texas to Arizona, northern states of Mexico. 
Secloporus reifi Ioosum Yarrow.

secloporw refidaram Yauron.

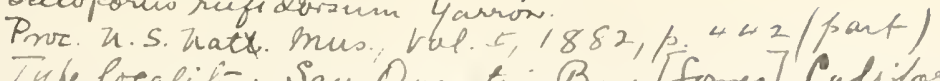

Tyhe Poeality. San Quen ki Bay, [Sorres]' Coliforncic.

Raupe: Hesteru Loner Califorma and Cerros Seacul. 
Sculapormo wordi Stajreger.

S.7lovid Pine Rigan.

Sceloparm woodi Stejuege, Proc. Biol. Soc. Wask, Vol. 31 , gune 29,1918,

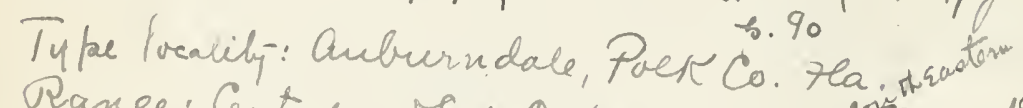

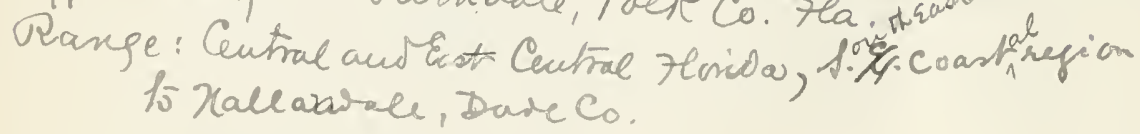


$\checkmark$ Sceloporus undulatus (Latreille)

PINE LIZARD, FENCE LIZARD

Stellio undulatus LATREILLE

Hist. Nat. Rept., Vol. ․ 1809, p. 40.

Sceloporus undulatus WiEgMaNN

Isis, 1828, p. 369.

Secloporus undulatus (part) COPE

Ann. Rep. U. S. Nat. Mus. 1898 (1900), p. 368, fig. 5\%.

TYPE LOCALITY: "Les grands bois de la Caroline."

RA NGE: Eastern States, New Jersey to Florida.

Sceloporus variabilis WIEgmanN

Sceloporus rariabilis Wiegmann

Herp. Mexico, 183t, p. 51.

Sceloporus rariabilis COIE

Ann. Rep. U. S. Nat. Mus. 1898 (1900), p. 398, fig. 67.

TYPE LOCALITY: Mexico.

RANGE: Guatemala and Mexico to southern Texas.

Sceloporus zosteromus COPE

Sceloporus zosteromus COPE

Proc. Ac. Nat. Sei. Phila., 1863, p. 105.

Sceloporus zosteromus COPE

Amm. Rep. U. S. Nat. Mus. 1898 (1900), p. 356, fig. 53.

TYPE LOCALITY: Cape St. Lucas, Lower California.

RA NGE:, Lower California.

Soriberse ard centrat

Phrynosoma (GENUS)

Wiegmane, Isis, 18:8, p. $36 \tau$

TYPE: orbicularis

\section{Phrynosoma blainvillii GraY}

Phrynosoma blainvillii GRAY

Zoöl. Beachey's Voyage, 1839, p. 96, pl. 99, fig. 1.

I'hrynosoma blaimillei (part) COPE

Aun. Rep. U.S. Nat. Mus. 1898 (1900), p. 493, fig. 74.

TYPE LOCALITY: California.

RANGE: Southern California, northern Lower California.

Phrynosoma brevicornis Boulenger

Phrynosoma brevicornis BOULENGER

Proe. Zool. Soc. London, 1916, p. 537, pl. 1.

TYPE LOCALITY: TeXas.

RANGE' : Unknown.

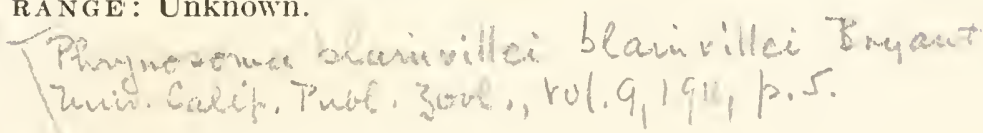


Phrynosoma brevirostre (GIRARD)

Tapaya brevirostris GIRARD

U. S. Expl. Exp., Herp., 1858, p. 397.

Phrynosoma brevirostre COPE

Proc. Ac. Nat. Sci. Phila., 1866, p. 309.

TYPE LOCALity: Plains of Kansas and Nebraska.

RANGE: Wyoming, the Basins of the Yellowstone and Platte Rivers.

\section{Phrynosoma cerroense Stejneger}

Phrynosoma cerroense STEJNEGER

N. Amer. Kauna, No. 7, May 91,1893, p. 187.

Phrynosoma cerroense COPE

Ann. Rep. U. S. Nat. Mus. 1898 (1900), p. 428, fig. 75.

TYPE LOCALITY : Cerros Island, Lower California. RANGE: The type locality.

ᄂ Phrynosoma cornutum (HARLAN)

Agama cornuta HarLaN

Journ. Ac. Nat. Sci. Phila., Vol. 4, 1825, p. 299, pl. 20.

Phrynosoma cornutum GraY

Griffith's Animal Kingdom, Syn. Rept., 1831, p. 9.

Phrynosoma cornutum COPE

Ann. Rep. U. S. Nat. Mus. 1898 (1900), p. 432, fig. 77.

TYPE LOCALITY: Great Plains east of the Rocky Mountains.

RANGE: From Kansas to the northern states of Mexico, west to

Colorado and New Mexico.

1. Phrynosoma coronatum (Blainville)

Agama (Phrynosoma) coronata B LAINville

Nouv. Ann. Mus. Hist. Nat. Paris, Vol. 4, 1835, p. 284, pl. 25, fig. 1.

Phrynosoma coronatum Duméril and Bibron

Erp. Gén., Vol. 4, 1837, p. 318.

Phrynosoma coronatum COPE

Ann. Rep. U. S. Nat. Mus. 1898 (1900), p. 430.

TYPE LOCALITY: "California” by inference.

RANGE: Lower California.

Phrynosoma ditmarsi STEJNEGeR

Phrynosoma ditmarsi StEJNEGer

Proc. U. S. Nat. Mus., Vol. 29, Feb. 2, 1906, p. 565.

TYPE LOCA LITY : State of Sonora, Mexico, not far from boundary of Arizona.

RANGE: Northern Mexico and probably southern Arizona. 
Phrymosma deuglassii ornatum (Irius).

Phrezursuma ornatum. Inend

liesp. in. s. Expl. Exp. pl. $2 q$, fi. 1-4.

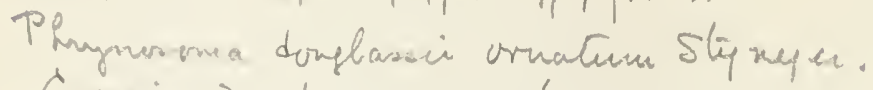

Coplia, ho.65,1919,p. H.

Tyine locatis: Salt Lare.

Rame: The saex fale Barin 
CHECK LIST N. AMER. AMPH. REPT.

Phrynosoma douglassii (BELL)

Agama douglassii BELL

Trans. Linu. Soc. London, Vol. 16, 1833, p. 105, pl. 10.

Phrynosoma douglasii WIEgMaxx

Herp. Mexico, 1834, 1). 54.

Phrynosoma douglasii douglassii COPE

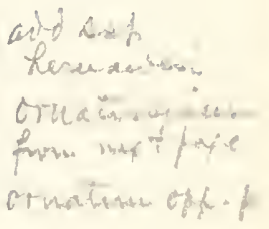

Ann. Rep. U. S. Nat. Mus. 1898 (1900), p. \$11, fig. 69.

TYPE LOCALITY: "In ora occidentali Americae Borealis ad ripas fluminis Columbiae."

RANGE: Oregon and Washington.

Lanivillei

$\checkmark$ Phrynosoma frontale VAx Deniurgh

Phrynosoma frontalis VAx Dexbergir

Proc. California Ac. Sci., Ser. 2. Vol. 4, 1894, p. 296.

Phrynosoma blainuillei (part) CoPE

Ann. Rep. U. S. Nat. Mus. 1898 (1900), p. 423.

Type Locality: Bear Valley, San Benito Co., California.

RANGE: El Dorado to Kern Counties, California.

donstasii

( Fhrynosoma heinandesi (Girard)

Tapaya hernandesi GIRARD

U. S. Expl. Exp., Herp., 1858, p. 395.

Phrynosoma hernandesi STEJNEger

N. Amer. Fauna. No. 3, 1890, p. 112.

Phrynosoma donglassii hernandesi COPE

Ann. Rep. U. S. Nat. Mus. 1898 (1900), p. 413, fig. 70.

TYPE LOCALITY: New Mexico.

RANGE: The plateau region of Colorado, Etah, New Mexico sedichow Mexico

Phrynosoma m'callii (Halloweli)

Anota m'callii Halloweli

Proc. Ac. Nat. Sci. Phila., Vol. 6, 1852, p. 182.

Phrynosoma maccallii Cope

Proc. Ac. Nat. Sci. Phila., 1866, p. 310.

Phrynosoma maccallii COPE

Ann. Rep. U. S. Nat. Mus. 1898 (1900), p. 448, fig. 8?.

TYPE LOCALity: Colorado Desert between Vallecita [sic] and

Camp Yuma, 160 miles east of San Diego, California.

RANGE: Southeastern California and southwestern Arizona.

\section{Phrynosoma modestum GiR.ARD}

Phrynosoma modestum Girard

Stansbury's Expl. Surv. Vall. Great Salt Lake, 1852, pp. 361, 365, pl. 6, figs. $4-8$. 
Anota modesta Cope

Ann. Rep. U. S. Nat. Mus. 1898 (1900), p. 437, fig. 78.

type locality: The Rio Grande, west of San Antonio, Texas, and from between San Antonio and El Paso.

RANGE: Texas to Arizona and northern Mexico.

Phrynosoma ornatissimum (GIrard)

Tapaya ornatissima GiRARD

U. S. Expl. Exp., Herp., 1858, p. 396.

Phrynosoma ornatissimum STEJNEGER

N. Amer. Fauna, No. 3, 1890, p. 115, pl. 12, figs. 3, a-c.

Phrynosoma douglassii ornatissimum COPE

Ann. Rep. U. S. Nat. Mus. 1898 (1900), p. 415, fig. 71.

TYPE LOCALITY: Mountainous region of New Mexico.

RANGE: Deserts of Arizona, New Mexico and Colorado, Sdaho,

herate and Utapis

Phrynosoma platyrhinos Girard

Phrynosoma platyrhinos Girard

Stansbury's Expl. Surv. Vall. Great Salt Lake, 1859, pp. 361, 363, pl. 7, figs. 1-5.

Anota platyrhina Cope

Ann. Rep. U.S. Nat. Mus. 1898 (1900), p. 443, fig. 81.

TYPE LOCAIJIT: Great Salt Lake Valley, Utah.

RANGE: Washington southward to California, Utah, Idaho and

Nevada, Sonth wectem Asejena and novih Easteme

fower Cafitumion?

Phrynosoma solare GraY

Phrynosoma solaris Gray

Cat. Liz. Brit. Mus., 1845, p. 229.

Phrynosoma solare Cope

Ann. Rep. U. S. Nat. Mus. 1898 (1900), p. 420, fig. 73.

TYPE LOCALITY : "California."

RANGE: Northern Lower California and extreme southern Ari-

zonaand Somerear.

\section{ANGUIDAE (FAMILY) \\ Ophisaurus (GENUS)}

Daudin, Bull. Soc. Philom. Paris, Vol. 3, No. 72, March, 1803, p. 188

TY PE: ventralis

Ophisaurus ventralis (LiNNÉ)

GLASS-SNAKE, JOINT-SNAKE

Anguis ventralis Linné

Syst. Nat., Ed. 12, 1766, p. 391. 
- Phrynosonea schmicti Backow

Phipnosoma schmicti. Batrom,

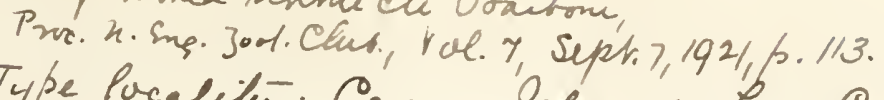

Type Pocalily: Cerrs Seace d, Lorra Calijormia.

Raupe: sacue.

Phrynosoma jamesi Schruidt

Phrynosoma fancwi Cohiuk t

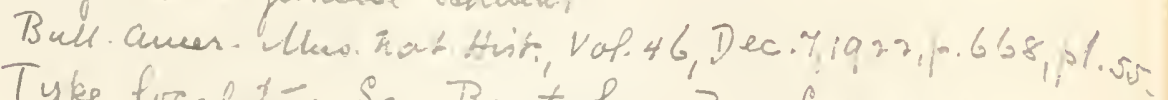

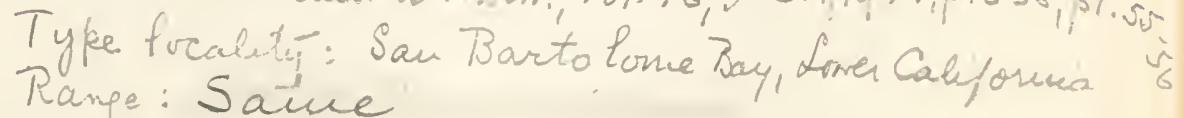

Ranpe: Saune

Plingmoverare nchoon Schuidt

Phrynosoma neleori sollewill-

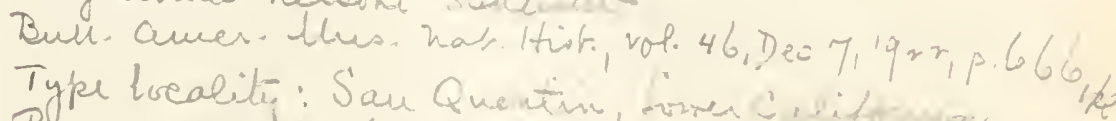

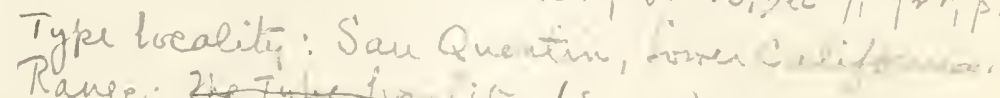
Range: 2ir Tylu. 


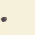


Ophisaurus ventralis DAUDIN

Hist. Nat. Rept. Vol. 7, 1803, p. 35\%, pl. 88.

Ophisaurus ventralis CoPE

Ann. Rep. U. S. Nat. Mus. 1898 (1900), p. 494, fig. 88.

TYPE LOCALITY: Carolina.

RANGE: Southern United States, in the east distributed northward to the Carolinas and Tennessee, in the central valley to Wisconsin, westward to New Mexico and southward to the state of Vera Cruz, Mexico.

\section{Gerrhonotus ( $\left.G E N L^{\top} S\right)$ \\ WIEG.IANN, Isis, 18\%8, p. 379 \\ TYPE: tessellatus = liocephalus}

Gerrhonotus coeruleus WIEGMANN ${ }^{1}$

Gerrhouotus coeruleus WiEguaxN

Isis, 1828, p. 380.

Gerrhonotus burnettii Cope

Ann. Rep. U. S. Nat. Mus. 1898 (1900), p. 526, fig. 94.

TYPE LOCALITY: "Brasilia” (in errore; probably San Francisco, Cal.).

RA NGE: Coast region of southern California.

Gerrhonotus infernalis (BAIRD)

Gerrhonotus infernalis BAIRD

Proc. Ae. Nat. Sei. Phila., 1858, p. 955.

Gerrhonotus lioeephalus infernalis COPE

Ann. Rep. U. S. Nat. Mus. 1898 (1900), p. 517, fig. 91.

TYPE LOCALITY: Devil's River, Texas.

RANGE: Southern Texas and northern Mexico.

$\checkmark$ Gerrhonotus multi-carinatus (Blainville)

Cordylus (Gerrhonotus) multi-carinatus BlaINville

Nouv. Aun. Mus. Hist. Nat. Paris, Vol. 4, 1835, p. 989, pl. 95, fig. … Gerrhonotus multicarinatus Duméril and Brbron

Erp. Gén., Vol. 5, 1839, p. 404.

Gerrhonotus multiearinatus (part) COPE

Ann. Rep. U. S. Nat. Mus. I898 (1900), p. 520.

TYPE LOCALITY: “ California."

RANGE : Lower California and Hexioa.

Gerrhonotus nobilis (BAIrD and Girard)

Elgaria nobilis Baird and GIRARD

Proc. Ac. Nat. Sei. Phila., Vol. 6, 1859, p. 129.

1 Type examined by L. Stejneger in Berlin.

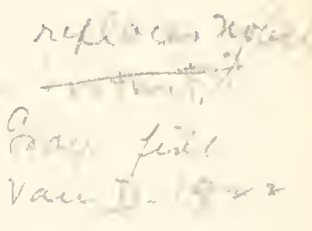


Gerrhonotus nobilis BAIRD

U. S. Mex. Bound. Surv., Vol. 2, 1859, Rept. p. 11, pl. 25, figs. 1-8. Gerrhonotus nobilis $\mathrm{COPE}$

Ann. Rep. U. S. Nat. Mus. 1898 (1900), p. 519 , fig. 92.

TYPE LOCALITY: Fort Webster, copper mines of the Gila (Santa

Rita del Cobre) New Mexico.

RANGE: New Mexico, Arizona and northern Mexico.

\title{
Gerrhonotus palmeri (STEJNEGER)
}

Gerrhonotus scinciauda palmeri STEJNEGER

N. Amer. Fauna, No. 7. May 31, 1893, p. 196.

Gerrhonotus palmeri $\mathrm{V}_{\text {an }}$ Denburgh

Occ. Papers California Ac. Sci., No. 5, 1897, p. 113.

Gerrhonotus multicarinatus palmerii COPE

Ann. Rep. U. S. Nat. Mus. 1898 (1900), p. 595.

TYPE LOCALITY: South Fork King's River, California.

RANGE: Sierra Nevada slopes, in central California.

$\checkmark$ Gerrhonotus principis (BAIRD and GIRARD)

Elgaria principis $\mathrm{B}_{\mathrm{AIRD}}$ and GIRARD

Proc. Ac. Nat. Sci. Phila., Vol. 6, 1852, p. 175.

Gerrhonotus principis STEJNEgER

N. Amer. Fauna, No. 7, May 31, 1893, p. 197.

Gerrhonotus principis COPE

Ann. Rep. U. S. Nat. Mus. 1898 (1900), p. 529, fig. 95.

TYPE LOCALITY: Oregon and Puget Sound.

RANGE: - Western Washington and Oregon, northwestern Califormia and Vancouver Island.

\section{Gerrhonotus scincicauda (Skilton)}

Tropidolepis scincicauda SKILTON

Amer. Journ. Sci. Arts, Ser. 2, Vol. 7, 1849, p. 202, pl. at p. 312, figs. 1-3.

Gerrhonotus multicarinatus (part) COPE

Ann. Rep. U. S. Nat. Mus. 1898 (1900), p. 520, fig. 93.

TYPE LOCALITY: "Dalles of the Columbia."

RANGE: Oregon and California west of the Sierra Nevada.

\author{
Barissia $(G E N U S)$ \\ Gray, Cat. Liz. Brit. Mus., 1845, p. 54 \\ TYPE: imbricata
}

Barissia levicollis SteJneger

Barissia levicollis StEJNEger

Proc. U. S. Nat. Mus., Vol. 13, Sept. 9, 1890, p. 184. 
$\checkmark$ Terrhonolis ocincicauda webbii (Rain),

topalow Serrhonohis rebbii Bairo, Proc Acad. hat Sci. Phila, $1858, p .255$. Terrhonolis, ecincicanda rrebbii Irinnell tCamp. unir. Calif. Publ. Zool, vol. $17,710.10,1917,168$ Jyhe locality: Sau Diefo, Calformia.

Range: Soñheru Califor hop Lomes Calif

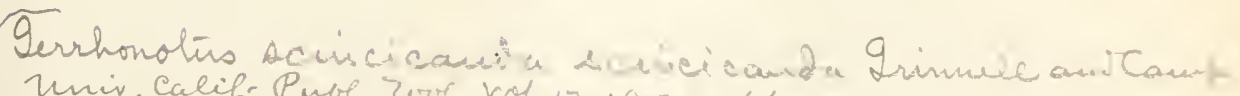
univ. Calif Pube, zove Vol.17,19.7,1,166 

Barissia levicollis Cope

Am. Rep. U. S. Nat. Mus. 1898 (1900), p. 53.5, fig. 96.

TYPE LOCALITY: "Mexican Boundary."

RANGE: Unknown.

ANNIELLIDAE (FAMILY)

Anniella $(G E N C S)$

Gray, Ann. Mag. Nat. Hist., Ser. 2, Vol. 10, 1852, p. 440

TYPE: pulchra

Anniella nigra (FIschen)

Aniella nigra Fischer

Ablı. Naturw. Ver. Hamburg, Vol. 9, Pt. 1, 1886, p. 9.

Anniella pulchra var. A. nigra Cope

Ann. Rep. U. S. Nat. Mus. 1898 (1900), p. (675.

TYPE LOCALITY: San Diego, California, probably in errore.

RANGE: Country about Pacific Grove, California.

Anniella pulchra GRAY

Anniella pulchra Gray

Ann. Mag. Nat. Hist., Ser. 2, Vol. 10, 185, p. 440.

Anniella pulchra COPE

Ann. Rep. U. S. Nat. Mus. 1898 (1900), p. 674, fig. 138.

TYPE LOCALITY: California.

RANGE: Coastal region of southern California.and nom the the Cower Careitgomperar.

\section{HELODERMATIDAE (FAMILY)}

Heloderma ( $G$ EN $U S)$

WIEGMANN, Isis, 1829 , p. 624

TYPE: horridum

\section{Heloderma suspectum Cope}

GILA MONSTER

IIcloderma suspectum COPE

Proc. Ae. Nat. Sei. Phila., 1869, p. 5.

IIeloderma suspectum Cope

Ann. Rep. U. S. Nat. Mus. 1898 (1900), p. 476, fig. 87.

TYPE LOCAlity: Sierra de la Union, Arizona, ("Sonora"). RANGE: Extreme southern Utah and Nevada, Arizona and So-

nora. 


\section{XANTUSIIDAE (FAMILY)}

Xantusia $(G E N U S)$

Baind, Proc. Ac. Nat. Sci. Plita.. 18.58, p. 255

TYPE : vigilis

\section{Xantusia gilberti Van Denburgh}

Xantusia gilberti $V_{\text {AN }}$ Denburgh

Proc. California Ac. Sci., Scr. 2. Vol. j. 1895, p. 121, pl. 11. Amocbopsis gilbertii COPE

Ann. Rep. U. S. Nat. Mus. 1898 (1900), p. .555, fig. 101.

TyPe locality: San Francisquito, Sierra Laguna, Lower California.

RANGE: Limits of range-unknown. Sovtheres fomer Califormic.

Xantusia henshawi STEunEger

Xantusia henshawi STEJNEGER

Proc. U. S. Nat. Mus. Vol. 16. July 21, 1893, p. 467.

Zablepsis henshavii COPE

Ann. Rep. U.S. Nat. Mus. 1898 (1900), p. 553, fig. 100.

TyPe locality: Witch Creek, San Diego Co., California.

RANGE: Known from a few localities in southern California.

Xantusia riversiana Cope

Xantusia riversiana $\mathrm{COPE}$

Proc. Ac. Nat. Sci. Phila., 1883. p. 99.

Xantusia riversiana COPE

Ann. Rep. U. S. Nat. Mus., 1898 (1900), p. 550, fig. 99.

TYPE LOCALITY: California, according to Cope, but later stated

by Prof. Rivers to have been San Nicholas Island, off the California Coast.

RANGE: San Nicholas, Santa Barbara and San Clemente Islands, Coast of California.

\section{Xantusia vigilis BAIRD}

Xantusia vigilis BAIRD

Proc. Ac. Nat. Sci. Phila., 1858, p. 255.

Xantusia vigilis Cope

Ann. Rep. U. S. Nat. Mus. 1898 (1900), p. 545. fig. 97.

TYPE LOCALitY: Fort Tejon, California.

RANGE: The tree-yucea zone of southern Nevada and southeastern Californiarano novth Eastem hover Califormia. 

$\checkmark$ Verticaria eacrulea Dicleveon Verticaria caerulea Dickersom.

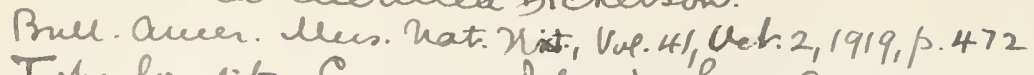
Type lvealit5. Carmen Drlased, Sorror California. Rampe: sacue.

Verticaria picta Van Deuburgh + Slevin. Verticaria pricta Vandenburgh. is Sevin Prove. Calif. Acad. Sei., Ser. 4, Vol.11. July S0, 1921, p.98. Type tucality: Monserrate bland, Dore. Cacif. Rauge: Sacue.

1 Vaticarin ceralkensis rand deubuph + Slerin Verticaria ceracbensis Yundeuburgh + Slerm. Pno. Calif. Cuas. Sci. Ser.4, Vop.11, Dec.17, 1921,0.396 Thu frabit: Cerallo Dlaut, Smer California. Raye: Sanu.

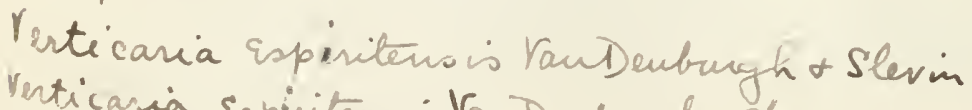
Venticaria Espiriteros Vai Deuburch + Slesin.

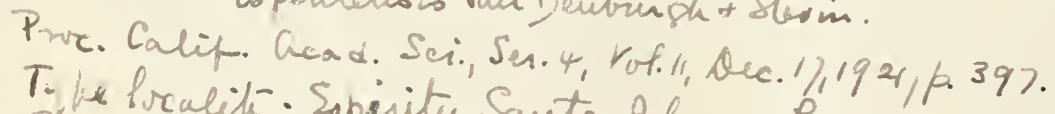
T. We brality: Sopiritu Sauto Blaud, Dorre Califorria. bisyc: Same.

Vuticaria hyperytha schmidti Vard entursh + Slevin

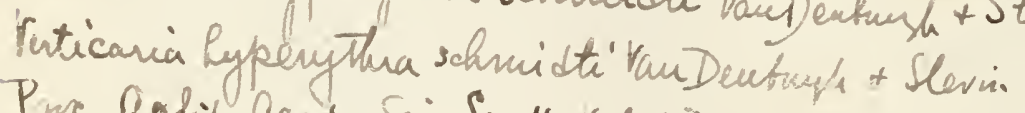

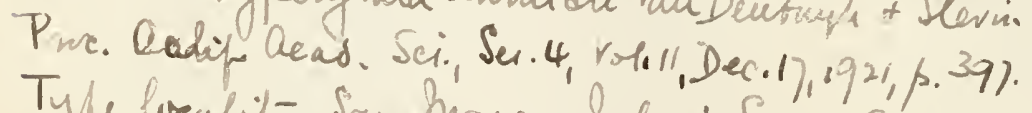

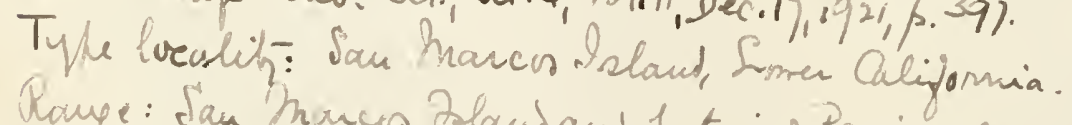
Raupe: Sau hunces Slaubant prterin of Peninsula of Rnu Califomia, opporit.

Verticani franciscensis Tandeubugh + Alevin Verticania Francicensis raudenbush + Slerin

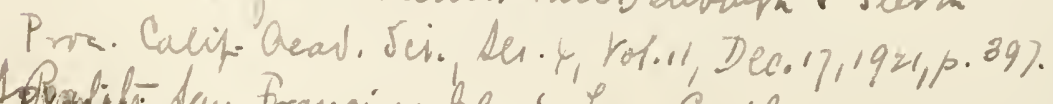

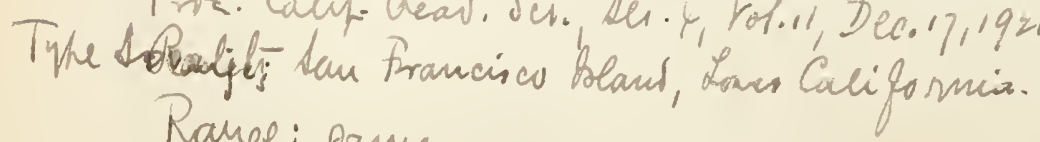
Raupe: oame. 
CHECK LIST N. AMER. AMPH. REPT.

\section{TEIIDAE $(F A M I L Y)$ \\ Verticaria (GENUS)}

Cope, Proc. Amer. Plilos. Soc., Vol. 11, 1869, p. 158

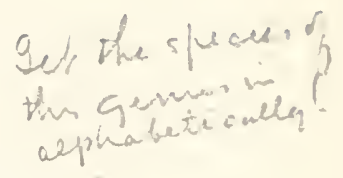

TYPE: hyperythra

\section{$\int$ Verticaria hyperythra hyperythra (COPE) \\ Verticaria hyperythra COPE \\ Proc. Amer. Philos. Soc., Vol. 11, 1869, p. 158. \\ Ierticaria hyperythra (part) Cope \\ Ann. Rep. U. S. Nat. Mus. 1898 (1900), p. 563.}

TyPE Locality: Cape San Lucas, Lower California.

RANGE: Southern end of Lower California.

\section{Verticaria hyperythra beidingi (STEJNEGER)}

Verticaria beldingi STEJNEGER

Proc. U. S. Nat. Mus., Vol. 17, May 4. 1S94, p. 1\%.

Verticaria hyperythra beldingi VAN DENBURGH

Proc. California Ac. Sci.. Ser. Q, Vol. 5, 189j. p. 131.

Verticaria hyperythra (part) COPE

Ann. Rep. L. S. Nat. Mus. 1898 (1900), p. 563, fig. 102.

TYPE LOCALITY: Cerros Island, Lower California.

RANGE: Southern California to middle Lower California, Cerros

Islands. Saut Mare orita and Magdelesa

Verticaria sericea VAx Denbtrgh

Verticaria sericea VAx Dexburgh

Proc. California Ac. Sci., Ser. ․, Vol. 5, 1895, p. 132, pl. 12.

Verticaria sericea COPE

Ann. Hip U. S. Nat. Mus. 1898 (1900), p. 564, fig. 103.

TYPE L OCALITY: San José Island, Lower California.

RA NGE: San José Island, Lower California.

\section{Cnemidophorus $(G E N U S)$ \\ WAgler, Syrst. Amph., 1830, p. 154 \\ TX $\mathrm{PE}$ : murinus \\ THE SWIFTS OR WHIP-TAILS}

Cnemidophorus arizonae $V_{\text {AN DeNBUrgh }}$

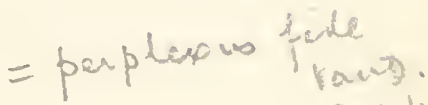

Cnemidophorus arizonae VAN DENBURGH

Proc. California Ac. Sci., Ser. 2, Vol. 6, p. 344.

Ty PE locality: Fairbank, Cochise Co., Arizona.

RANGE: Southern Arizona. 
Cnemidophorus grahamii BaIrd and Girard

Cnemidophorus grahamii BAIRD and GIRARD

Proc. Ac. Nat. Sci. Phila., Vol. 6, 1852, p. 128.

Cnemidophorus grahamii grahamii CoPE

Ann. Rep. U. S. Nat. Mus. 1898 (1900), p. 598, fig. 117.

ty

RANGE: Texas.

1) Cnemidophorus labialis StEJNEGer

Cnemidophorus labialis STEJNEGER

Proc. U. S. Nat. Mus., Vol. 12, June, 1890, p. 643.

Cnemidophorus labialis COPE

Ann. Rep. U. S. Nat. Mus. 1898 (1900), p. 610, fig. 122.

TY PE LOCALITY: Cerros Island, Lower California.

RANGE : Cerros Island, Lower California.

\section{Cnemidophorus martyris STEJNEGER}

Cnemidophorus martyris STEJNEGER

Proc. U. S. Nat. Mus., Vol. 14, Aug. 31, 1891, p. 407.

type Locality: San Pedro Martir Island, Gulf of California.

RANGE: Type locality.

Cnemidophorus maximus Cope

Cnemidophorus maximus COPE

Proc. Ac. Nat. Sci. Phila., 1863, p. 104.

Cnemidophorus maximus CoPE

Ann. Rep. U. S. Nat. Mus. 1898 (1900), p. 570, fig. 104.

TyPE LOCALity: Cape St. Lucas, Lower California.

RANGE: Southern Lower California.

\section{Cnemidophorus melanostethus COPE}

Cnemidophorus melanostethus COPE

Proc. Ac. Nat. Sci. Phila., 1863, p. 104.

Cnemidophorus tessellatus melanostethus CoPe

Amn. Rep. U. S. Nat. Mus. 1898 (1900), p. 581, fig. 109.

TYPE LOCALity: " Region of the Colorado of California" Colorado Desert, Arizona.

RANGE: Deserts of southern Arizona.

\section{Cnemidophorus multiscutatus (COPE)}

Cnemidophorus tessellatus multisentatus CoPE

Trans. Amer. Philos. Soc., Vol. 7, 1892, p. 38.

Cnemidophorus multiscutatus VAN Denburgu

Proc. California Ac. Sci., Ser. 4 , Vol. 4, 1914, p. 144.

Cnemidophorus tessellatus multiscutatus COPE

Ann. Rep. U. S. Nat. Mus. 1898 (1900), p. 586, fig. 111. 
- Cremidophorus celeripes Dickerson.

Enemi dophorus celeripes Dickerson.

Bnll. Anver. Uns. hat. Kist., rol. 41, Ger. 2, 1919, p. 472-3

Type localit;: San Gozé Bslaud, Lorre Califarnia.

TEanc $c$ : Lave

Cnemidophorus dis parilis Dicterson.

Cnenidophorus disparilis Diekerson.

Bull. Quver. llus. hat. Hist., Vol. 41,0 ch: 2, 1919, p. 473-4.

Tyne lvealits: Ti buron Bland, fuly of Califomia.

Raype: same.

$\checkmark$ Cremidophorus estebanenais Diellevor.

Cnemi dophorers estebanensis Diexersom.

Bull. Anver. Mus. hat. Hist, vol.41, bech 2, 1919, p. 474-5.

Tyre brealits: Sau Eteban kland, Sulf of Califonia. Raye: sacue.

$\checkmark$ Cnenidophorus punctilinealis Dicherson. funsein

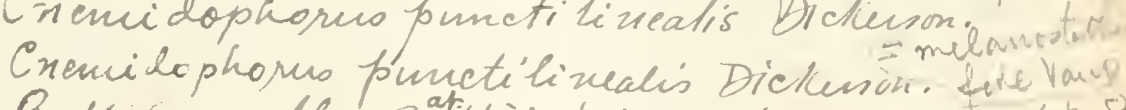

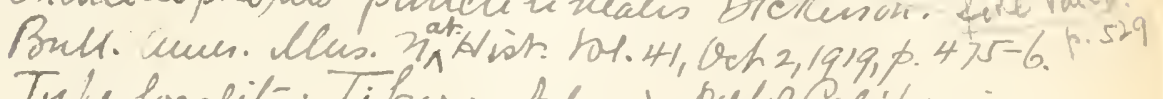

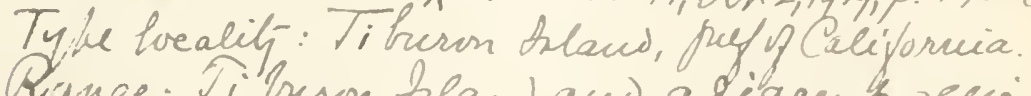
Rinpe: Jilinon bland aud a \&jacent sgeion i) Sorura (fuaymas).

Cneniclophorus farto lomas dicterson. Cnenilophorus barto-lomas Dickeroon.

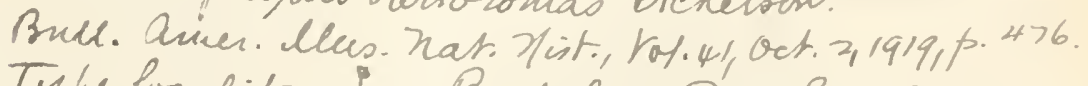
Tyle localits: San Bartolome Bray, Lorrer Califorria. Rane: Same.

Cnemidophores catalinensis raud Deubugh + Sherin Cremidophomes catalinensis tandenbugh + Slerin.

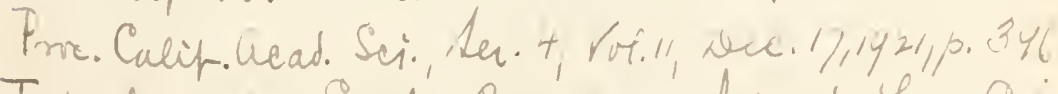
Type lvadif-: Sauta Cutalina blant, Lomer Californier Ravye: Janue. 
Cremidophorus bacalis VauDeubursh + Slevn:

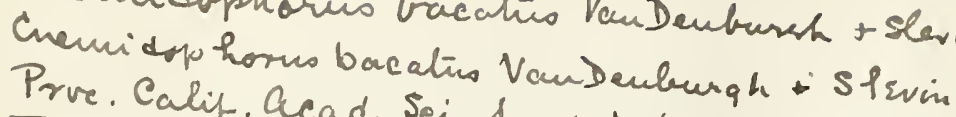

Prve. Calif. Acad. Sei., Ser. 4 , Vol. 9, July30, 1921, p. 97.

Typelveality: Sau Pedro holaseo Slaro, Lover California

Rauge: Same.

Creni tophorus canus Vau Deuburgh s Slevim

Cremidophorus canus Vau Deubungh + Slevon

Proe. Cacif. Qcad. Sci., Ser. 4, top. 9, July30,1911,1.97.

Tyle Prealit-: Sal Si Puedes doland, Lover Califormia

Rauge: daune and $x$. S. San tiremg the

1 Cnemidopkorus dickersonae Tau Deuburgh. Slevin

Cremidophorrs dieknsonar Van Denbugh + Slevin Proc. Calif. Acad. Sei, Ser.4, top. 9, Jucly $30,1921,0.97$

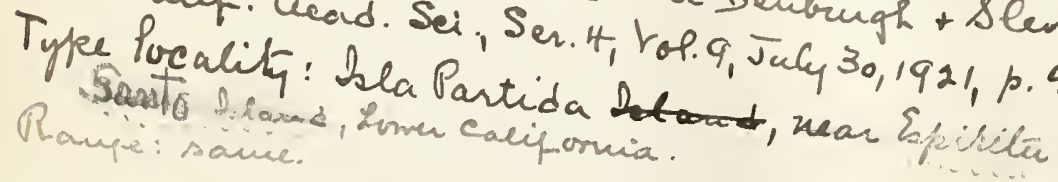

Ravipe: saine. 
TYPE LOCAyitr : Cepros Island, Lower California.

RA NGE: Cefros Island, Lower California.

Cnemidophorus perplexus BAIRD and Girais)

Cnemidophorus perplexus BaIrd and Girard

Proc. Ac. Nat. Sci. Phila. Vol. 6, 1852, p. 198.

Cnemidophorus tessellatus perplexus (part) Cope

Ann. Rep. U. S. Nat. Mus. 189S (1900), p. 573, fig. 106.

TYPE LOCALITY: Valley of the Rio San Pedro, tributary of the

Rio Grande del Norte, Texas.

RANGE: 'Texas and New Mexico.

Cnemidophorus rubidus (Cope)

Cnemidophorus tessellatus rubidus Cope

Trans. Amer. Philos. Soc., Ser. 2, Vol. 17, 1892, p. 36, pl. 12, fig. f.

Cnemiácphorus rubidus VAx Denburgh

Proc. California Ac. Sci., Ser. \$, Vol. 4, 1914, p. 145.

Cnemidophorus tessellatus rubidus Cope

Ann. Rep. U. S. Nat. Mus. 1898 (1900), p. 584, fig. 110.

TYPE LOCALity: Sta. Margarita Island, Lower California.

RANGE: Sta. Margarita Island, Lower California. awe handele ha

\section{Cnemidophorus sexlineatus (Linvé)}

Lacerta sexlineata LıNNé

Syst. Nat. Ed. 12, 1766, p. 364.

Cnemidophorus sexlineatus Duméril and Bibron

Erp. Gén., Vol. 5, 1839, p. 131.

Cnemidophorus sexlineatus Cope

Ann. Rep. U. S. Nat. Mus. 1898 (1900), p. 593, fig. 116.

TYPE LOCALITY: In Carolina.

RANGE: Maryland to Florida, west to northern Mexico and Arizona and up the Mississippi Valley as far north as Lake Michigan.

Cnemidophorus gularis BAIRD and Girard

Cnemidophorus gularis BAIrD and GIRARD

Proc. Ac. Nat. Sci. Phila., Vol. 6, 1852, p. 128.

Cnemidophorus gularis gularis COPE

Ann. Rep. U. S. Nat. Mus. 1898 (1900), p. 602, fig. 118.

ty la locality: Indianola and San Pedro River, Texas.

RANGE: Arkansas and Oklahoma through Texas west to Arizona also the northern states of Mexico.

Cnemidophorus tessellatus tessellatus ( $\mathrm{SAY}$ )

Ameiva tesselata $\mathrm{S}_{\mathrm{AY}}$

Long's Exp. Rocky Mts., Vol. 2, 1823, p. 50. 
Cnemidophorus tessellatus BAIRD

Pacific R.R. Surv., Vol. 10, Pt. 4, 1859, p. 18.

Cnemidophorus tessellatus tessellatus (part) COPE

Ann. Rep. U. S. Nat. Mus. 1898 (1900), p. 575, fig. $10 \%$.

TYPE LOCAlity: Arkansas River, near Castle Rock Creek, Colorado.

RANGE: Texas to California, also Utah, Colorado and Nevada.

Cnemidophorus tesseilatus mundus (CAMP)

Cnemidophorus tigris mundus CAMP

Univ. California Publ. Zoöl., Vol. 17, Dec. 28, 1916, p. 71.

TYPE LOCALITY: "Near Fort Yuma, in San Joaquim Valley"?

$=$ Fort Miller, Fresno County, California.

RANGE: Central California.

\section{Cnemidophorus tesseilatus stejnegeri (Van Denburgh)}

Cnemidophorus stejnegeri VAN DenBURGH

Proc. California Ac. Sci., Ser. 2, Vol. 4, 1894, p. 300.

Cnemidophorus grahamii stejnegerii CopE

Ann. Rep. U. S. Nat. Mus. 1898 (1900), p. 599.

TYPE LOCALITY : Near Ensenada, Lower California.

RANGE: Southern California and northern Lower California.

\section{SCINCIDAE}

\section{Leiolopisma ( $G E N U S)$}

Dumíril and Bibron, Erp. Gén., Vol. 5, 1839, p. 742

TYPE: telfairii

Leiolopisma laterale ( $\mathrm{S}_{\mathrm{AY}}$ )

Scincus lateralis $\mathrm{S}_{\mathrm{AY}}$

Long's Exp. Rocky Mts., Vol. 2, 1823, p. 324.

Leiolopisma laterale JoRDan

Man. Vertebr. North. U. S., Ed. 8, 1899, p. 201.

Liolepisma laterale COPE

Ann. Rep. U. S. Nat. Mus. 1898 (1900), p. 692, fig. 193.

TYPE LOCALITY: Banks of Mississippi River below Cape Girardeau, Missouri.

RANGE: Maryland to Florida, west to Illinois and Texas. ${ }^{1}$

1 What is apparently the same species occurs also in China and Formosa. 
valid fise Schinutat

= rubite, fire raud $19=6$

Cnemidophorus vandenburghi Dickerson.

Cnemidophorus vandenturghi Dielcerson.

Bull. Amer. Mus. hat. 2list., hol. 4, oct $2,1919,7.47)$

Type treatity: Carmen 81 . quet of Califurnia.

Range: soure 



\section{Plestiodon (GENUS) \\ Duméril and Bıвrox, Erp. Gén., Vol. 5, 1839, p. 697 \\ TYPE: quinquelineatus}

Plestiodon anthracinus BAIRD

Plestiodon anthracinus BAIRD

Journ. Ac. Nat. Sci. Phila., Ser. 2, Vol. 1, 1819, p. 294.

Eumeces anthracinus COPE

Ann. Rep. U.S. Nat. Mus. 1898 (1900), p. 661 , fig. 135.

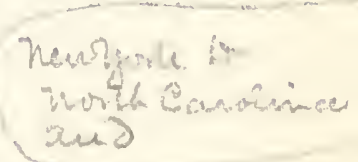

type Locality: North Mlontain near Carlisle, Pennsylvania.

RANge: Hestern Penpy yraniante Missouri, Arkansas and Texas.

Plestiodon brevilineatus (COPE)

Eumeces brevilineatus Cope

Bull. U. S. Nat. Mus.. No. 17, 1880, p. 18.

Eumeces brevilineatus Cope

Ann. Rep. L. S. Nat. Mus. 1898 (1900), p. 664, fig. 137.

TyPe locality: Helotes, Bexar Co., Texas.

RANGE: Texas.

\section{Plestiodon egregius BAIRD}

Plestiodon egregius BaInD

Proc. Ac. Nat. Sci. Phila., 1858, p. 256.

Eumeces egregius Cope

Ann. Rep. U. S. Nat. Mus. 1898 (1900), p. 655, fig. 192.

TYPE LOCALITY: Indian Key, Florida.

RANGE: Western Florida and the Florida Keys.

Plestiodon fasciatus (Livné)

BLLE-TAILED SKINK, SCORPION

Lacerta fasciata LiNxé

Sirst. Nat., Ed. 10, Vol. 1, 1758, p. 209.

Plestiodon fasciatus BAIRD

Journ. Ac. Nat. Sci. Phila., Ser. ̊,. Vol. 1, 1849, p. 294.

Eumeces quinquelineatus COPE

Ann. Rep. U. S. Nat. Mus. 1898 (1900), p. 632, fig. 125.

TYPE LOCALITY: Carolina.

RANGE: Southern New England to Florida, up the Mississippi

Valley to Canada and westward to Arizona.

Plestiodon guttulatus ${ }^{1}$ (Hallowell)

Lamprosaurus guttulatus HaLlowell

Proc. Ac. Nat. Sci. Phila., Vol. 6, 1852, p. 206. 
Plestiodon guttulatus Halloweli.

Proc. Ac. Nat. Sci. Phila., 1857, p. 215.

Eumeces guttulatus Cope

Ann. Rep. U. S. Nat. Mus. 1898 (1900), p. 645, fig. 127.

TyPE LOCALity : Fort Filmore below Jornada del Muerte, New Mexico.

RANGE: Western Texas and Oklahoma to Arizona.

\section{Plestiodon longirostris CoPE}

Plestiodon longirostris COPE

Proc. Ac. Nat. Sci. Phila., 1861, p. 313.

Eumeces longirostris $\mathrm{COPE}$

Ann. Rep. U. S. Nat. Mus. 1898 (1900), p. 631, fig. $12 t$.

TYPE LOCALITY: Bermuda.

RANGE: Bermuda.

\section{Plestiodon multivirgatus HaLloweld}

Plestiodon multivigatus Hallowell

Proc. Ac. Nat. Sci. Phila., 1857, p. 215.

Eumeces epipleurotus COPE

Ann. Eep. U. S. Nat. Mus. 1898 (1900), p. 650, fig. 129.

Eumeces leptogrammus Cope

Ann. Rep. U. S. Nat. Mus. 1898 (1900), p. 65 l, fig. 130.

Eumeces multivirgatus Cope

Ann. Rep. U. S. Nat. Mus. 1898 (1900), p. 653, fig. 131.

TyPe Locality: Posa Creek, 460 miles west of Fort Riley, Kansas.

RANGE: Nebraska to Northern Texas.

\section{Plestiodon obsoletus Baird and Girard}

Plestiodon obsoletum BAIRD and GIraRD

Proc. Ac. Nat. Sci. Phila., Vol. 6, 1852, p. 129.

Eumeces obsoletus Cope

Ann. Rep. U. S. Nat. Mus. 1898 (1900), p. 646, fig. 128.

TYPE LOCALITY: Valley of the Rio San Pedro, tributary of the

Rio Grande del Norte, Texas.

RANGE: Utah and Kansas southward to northern Mexico.

\section{Plestiodon pachyurus (COPE)}

Eumeces pachyurus Cope

Bull. U. S. Nat. Mus., No. 17, 1880, p. 19.

Eumeces pachyurus COPE

Ann. Rep. U. S. Nat. Mus. 1898 (1900), p. 659.

TYPE LOCALITY: Near Dallas, 'Texas.

RANGE: Texas. 


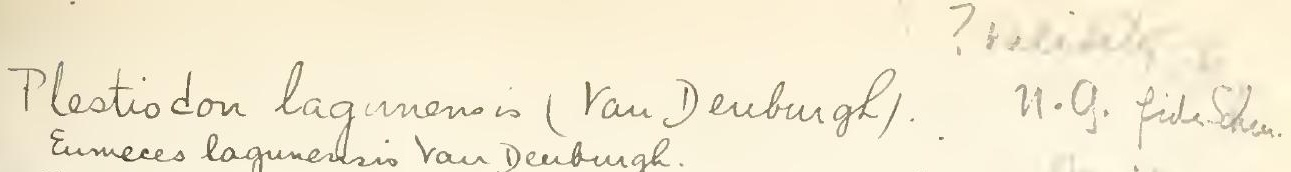
Enmeces laguneshsis Vau Deuburgh. Proc Calif. Acad. Sci, Ser. 2, rol. $5,1895, p .134, p / .33$ Plestiodon lagmensis Vampenburgh + Slevin Prre. Cacif acad. Sei, Ses. 4, rol.11,1921,p.02.

Syle Preality: Sau 'Iranaisquito, Sierra Laguna, Forrer Californica. 

Plestiodon pluvialis (COPE)

Eumeees pluvialis COPE

Bull. U. S. Nat. Mus., No. 17, 1880, p. 19.

Eumeces pluvialis $\mathrm{COPE}$

Ann. Rep. U. S. Nat. Mus. 1898 (1900), p. 663, fig. 136.

TYPE LOCALITY: Mobile, Alabama.

RANGE: Central Gulf area.

\section{Plestiodon septentrionalis BAIRD}

Plestiodon septentrionalis BAIRD

Proc. Ac. Nat. Sci. Phila., 1858, p. 2566.

Eumeces septentrionalis COPE

Ann. Rep. U. S. Nat. Mus. 1898 (1900), p. 656, fig. 133.

TYPE LOCALITY: Minnesota and Nebraska.

RANGE: Minnesota, Nebraska and Kansas.

\section{Plestiodon skiltonianus BAIRD and GirARD}

Plestiodon skiltonianum $\mathrm{BAIRD}$ and GIRARD

Stansbury's Expl. Surv. Vall. Great Salt Lake, 1859, p. 349, pl. 4, figs. 4-6.

Eumeces sliltonianus COPE

Ann. Rep. U. S. Nat. Mus. 1898 (1900), p. 640, fig. 126.

TYPE LOCALITY: Oregon.

RANGE: Western Oregon, and most of California, except the ex-

treme southeastern desert area; Lower California; \&ai Twa

thone havade lis Weals."

Plestiodon tetragrammus BAIRD

Plestiodon tetragrammus BAIRD

Proc. Ac. Nat. Sci. Phila., 1858, p. 956.

Eumeces tetragrammus CopE

Ann. Rep. U. S. Nat. Mus. 1898 (1900), p. 660.

TYPE LOCALITY : Lower Rio Grande.

RANGE: Southern Texas and northeastern Mexico.

\section{Neoseps (GENUS)}

Stejneger, Proc. U. S. Nat. Mus., Vol. 39, Sept. 23, 1910, p. 33 TYPE: reynoldsi

Neoseps reynoldsi Stejneger

Neoseps reynoldsi STEJNEGER

Proc. U. S. Nat. Mus., Vol. 39, Sept. 23, 1910, p. 34.

ty Pe locality: Near Spring Lake, Fruitland Park, Lake Co.,

Florida.

RANGE : Central Florida. 


\section{BIPEDIDAE (FAMILY)}

Bipes ( $G E N U S)$

Latreille, Hist. Nat. Rept., Vol. 2, 1802, p. 90 TY PE: canaliculatus

Bipes biporus (COPE)

Euchirotes biporus CopE

Amer. Nat., Vol. 28, 1894, p. 436, figs. 5a-c.

Euchirotes biporus Cope

Ann. Rep. U. S. Nat. 1898 (1900), p. 680, fig. 140.

TyPE LOCAlity: La Paz, ${ }^{1}$ Lower California.

RANGE: Southern Lower California.

\section{LEPOSTERNIDAE (FAMILI')}

Rhineura ( $G E N U S)$

Cope, Proc. Ac. Nat. Sci. Phila., 1861, p. 75

TYP E : floridana

Rhineura floridana (BAIRD)

Lepidosternon floridanum $\mathrm{B}_{\mathrm{AIRD}}$

Proc. Ac. Nat. Sci. Phila., 1858, p. 255.

Rhineura floridana Cope

Proc. Ac. Nat. Sci. Phila., 1861, p. 75.

Rhineura floridana CoPE

Ann. Rep. U. S. Nat. Mus. 1898 (1900), p. 686, fig. 141. TYPE LOCALITY: Micanopy, Florida. RANGE: Peninsular Florida.

\section{SERPENTES (SUBORDER)}

Linné, Syst. Nat., Ed. 10, Vol. 1, 1758, p. 214

\section{LEP'TOTYPHLOPIDAE (FAMILY) \\ Leptotyphiops ( $G E N U S$ ) \\ Fitzinger, Syst. Rept., 18ł3, p. 94 \\ TY PE: nigricans}

Leptotyphlops dulcis (Barrd and Grrard)

Rena dulcis BA1RD and Girard

Cat. N. Amer. Rept., Pt. 1, 1853, p. 142.

1 Given in crror in original description as Cape St. Lucas. 


Leptotyphlops duleis StEJneged

Proc. U. S. Nat. Mus., Vol. 14, Oct. 31, 1891, p. 501.

Glanconia dulcis COPE

Ann. Rep. U. S. Nat. Mus. 1898 (1900), p. 717, fig. 143.

TyPe locality: Between San Pedro and Comanche Springs,

'Texas.

IR A NGE: Northern Mexico, Texas and New Mexico.

\section{Siagonodon ( $G E N U S)$}

Peters, Sitz. Ber. Ges. Naturf. Freunde, Berlin, 1881, p. 71 TYPE: septemstriatus

Siagonodon humilis (BaIr1) and Girard)

Rena humilis BAIRD and GIRARD

Cat. N. Amer. Rept., pt. 1, 185\%, p. 143.

Siagonodon humilis Van Denburgi

Occ. Pap. California Ac. Sci., Vol. 5, 1897, p. 150.

Glanconia humilis COPE

Ann. Rep. U. S. Nat. Mus. 1898 (1900), p. 719, fig. 144.

TYPE LOCALITY: Valliecitas (sic), California.

RANGE: Deserts of Arizona, southern California, Lower California and northwestern Mexico.

\section{BOIDAE (FAMILY)}

\section{Lichanure. ( $G E N U S)$}

Cope, Proc. Ac. Nat. Sci. Plila., 1861, p. 304

TYPE: trivirgata

Lichanura roseofusca CoPE

Lichanura roscof usca COPE

Proc. Ac. Nat. Sci. Plila., 1868, p. 2.

Lichanura roseof usca Cope

Ann. Rep. U. S. Nat. Mus. 1898 (1900), p. 724, fig. 146.

TyPE LOCALITY: Northern Lower California.

RANGE: Southern California, northern Lower California and Arizona.

\section{Lichanura trivirgata COPE}

\section{Lichanura trivirgata COPE}

Proc. Ac. Nat. Sci. Phila., 1861, p. 304.

Lichanura triviryata COPE

Ann. Rep. U.S. Nat. Mus. 1898 (1900), p. 723, fig. 145.

ty Pe locality : Cape St. Lucas, Lower California.

RANGE: Lower California. 
Charina ( $G E N U S)$

Gray, Cat. Snakes Brit. Mus., 1849, p. 113

TYPE: bottae

\section{Charina bottae (BLAINVILLE)}

RUBBER SNAKE, BALL SNAKE

Tortrix boltae Blainville

Nouv. Ann. Mus. Hist. Nat. Paris, Vol. 4, 1835, p. 289, pl. 26, figs. 1-B1. Charina bottae GraY

Cat. Snakes Brit. Mus., 1849, p. 113.

Charina bottae COPE

Rep. U. S. Nat. Mius. 1898 (1900), p. 798, fig. 148.

TYPE LOCALITY: California.

RANGE: Humid districts of California, Nevada, Idaho, Oregon

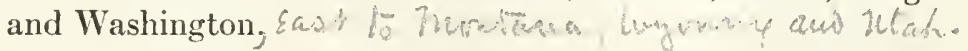

\section{COLUBRIDAE (FAMILY)}

Carphophis ( $G$ ENUS)

Gervais, D’Orbigny's Dict. Univ. Hist. Nat. Vol. 3, 1843, p. 191

TYPE: amoena

WORM SNAKES

Carphophis amoena ( $\mathrm{SAY}$ )

Coluber amoenus $\mathrm{S}_{\mathrm{AY}}$

Journ. Ac. Nat. Sci. Phila., Vol. 4, pt. , 1895, p. 237.

Carphophis amoena GervaIs

D’Orbigny's Dict. Univ. Hist. Nat., Vol. 3, 1843, p. 191.

Carphophiops amoenus COPE

Ann. Rep. U. S. Nat. Mus. 1898 (1900), p. 785, fig. 151.

TYPE LOCALITY: Pennsylvania.

RANGE: Connecticut to Florida, westward in Ohio, Illinois and Indiana.

\section{Carphophis vermis (KENNICOTT)}

Celuta vermis KenNicotT

Proc. Ac. Nat. Sci. Phila., 1859, p. 99.

Carphophiops vermis COPE

Ann. Rep. U. S. Nat. Mus. 1898 (1900), p. 737, fig. 159.

TYPE LOCALITY: Missouri.

RANGE: Kansas southward to Louisiana. 
$\checkmark$ Charmia bottac utahensis Vau Deubungl.

mit Charmia bottac wtak ensis randenbsesh.

onut Proc. Calif. Acad. Sei. Ser. 4, Vo1. 10, Auq. 6,1920, p. 31.

Type lvealib: fitche Cottonwood Cañon, Wasatch S. Wtah Rarege: Northwesterve Utah, also liake 



\author{
Abastor $(G E N U S)$ \\ Gray, Cat. Snakes Brit. Mus., 1849. p. 78 \\ TYPE: erythrogrammus
}

\title{
Abastor erythrogrammus (DAUDIN) \\ RAINIBOW SNAKE
}

Coluber erythrogrammus DAUDIN

Hist. Nat. Rept., Vol. 7, 1803, p. 93, pl. 83, figs. 2a-b. Abastor erythrogrammus GriY

Cat. Snakes Brit. Mus., 1849, p. 78.

Abastor erythrogrammus COPE

Ann. Rep. U. S. Nat. Mus. 1898 (1900), p. 738, fig. 159.

TYPE LOCALITY : Etats Unis d'Amérique.

RANGE: Virginia southward to Florida and Alabama.

\section{Farancia (GENUS)}

Gray, Zool. Mise., 1842, p. 78

TYPE: drummondi $=$ abacura

Farancia abacura (НоLвRоок)

HORN-SNAKE, MUD-SNAKE

Coluber abacurus HoLBrook

N. Amer. Herp., Ed. 1, Vol. 1, 1836, p. 119, pl. 23.

Farancia abacura BAIRD and GIrARD

Cat. N. Amer. Rept., Pt. 1, 1853, p. 193.

Farancia abacura COPE

Ann. Rep. U.S. Nat. Mus. 1898 (1900), p. 7t1, fig. 154.

TYPE LOCALITY: South Carolina.

RANGE: Virginia to Florida and Louisiana, in the Mississippi

Valley northward to Indiana.

\section{Diadophis ( $G E N U S)$}

Baird and Girard, Cat. N. Amer. Rept., Pt. 1, 1853, p. 112

TYPE: punctatus

RING-NECK SNAKES

Diadophis amabilis BAIRD and GIRARD

Diadophis amabilis BAIRD and GIRARD

Cat. N. Amer. Rept., Pt. 1, 1853, p. 113.

Diadophis amabilis COPE

Ann. Rep. U.S. Nat. Mus. 1898 (1900), p. 746, figs. 157, 160.

TYPE LOCALITY: San José, California.

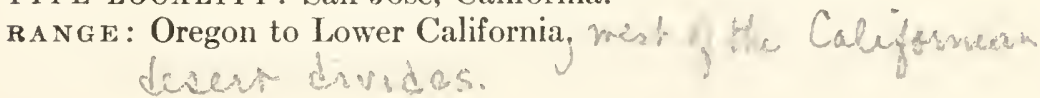


Diadophis arnyi (KENNICOTT),

Diadophis arnyi KenNicotT

Proc. Ac. Nat. Sci. Phila., 1859, p. 99.

Diadophis regalis arnyi COPE

Ann. Rep. U. S. Nat. Mus. 1898 (1900), p. 745, fig. 156.

TYPE LOCALITY: Hyatt, Anderson County, Kansas. n.W.

RANGE: Ohio, Illinois, Indiana, Kansas, Iowa, Missouri, Arkansas,

Oklahoma, and Texas.

\section{Diadophis punctatus (LINNÉ)}

Coluber punctatus LINNÉ

Syst. Nat., Ed. 12, Vol. 1, 1766, p. 376.

Diadophis punctatus BAIRD and GIRARD

Cat. N. Amer. Rept., Pt. 1, 1853, p. 112.

Diadophis punctatus COPE

Ann. Rep. U. S. Nat. Mus. 1898 (1900), p. 751, fig. 169.

TYPE LOCALITY: Carolina.

RANGE: In the east from southeastern Canada to Florida, in the west reaching Michigan also found in Illinois, Ohio and Tennéssee.

Diadophis regalis BAIRD and GIRAIiD

Diadophis regalis BAIRD and GIRARD

Cat. N. Amer. Rept., Pt. 1, 1853, p. 115.

Diadophis regalis $\mathrm{COPE}$

Ann. Rep. U. S. Nat. Mus. 1898 (1900), p. 744, fig. 155.

TYPE LOCALITY : Sonora, Mexico.

RANGe: Texas, New Mexico and Arizona to state of Vera Cruz,

Mexico.

\section{Heterodon $(G E N U S)$}

Latreille, Hist. Nat. Rept., Vol. 4, 1802, p. 32

TYPE: platirhinos $=$ contortrix

HOG-NOSE SNAKES

Heterodon browni STEJNEGER

Heterodon browni STEJNEGER

Proc. Biol. Soc. Washington, Vol. 16, 1903, p. 123.

TYPE LOCALITY: Lemon City, Florida.

RANGE: Extreme southern Florida.

Heterodon contortrix (LINNÉ) ${ }^{1}$

PUFFING ADDER, SPREADING ADDER

Boa contortrix Linne

Syst. Nat., Ed. 19, Vol. 1, 1766, p. 373.

1 This snake for years has been ealled $H$. platyrhinus or platirkinos but this name is clearly a synonym. Linné in the tenth editiou of the Systema Naturae (p. 216) deseribed Coluber constrictor. This 
$\checkmark$ Diadophis punctatus strictogenys Cope Diadophis punctatis var striclogenups Cofe Proc. Acizat. Sci. Ptila, 1860, p. 250.

Type fre ality: muscuom.

Raupe: S. ellinvis, thromph the forer hississiftic Valley to the fuep.

Diadophis punctalin punctátios (Limé) ..

Tyte Lrealily: Carolina Range; Itsifistates forlands ing 7 orida, north wand to northere nuth Carolina from sea-level to about 2500 Reet, possibly higher mi the monhtains of Sonth Carolina aced Gergia

Diabophis punctatus edwardsie (Merremi) Cohibcr ed wardsii Merren, Tent. Sypt.Rept., $1820, \% .136$

Type localily: Penusylvania.

tunge: The montaius of the Carolinas somfenvessee nonturaw to the Mnthern Penins nla of michigavis sonthern Cauara and the M aratiue Proriuces.

Dinnth The Opreachions

Diagothe punatali, functatin Bubrom

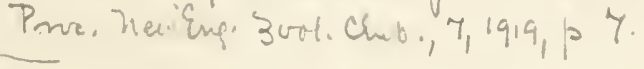

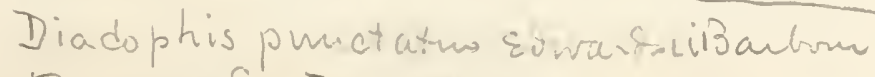
Proc. Teni he. Zrot. Club., 7, $1919,10.9$ 
Liopeltis vernalis Cope

Proc. Ac. Nat. Sci. Phila., 1860, p. 560.

Liopeltis vernalis Cope

Ann. Rep. U. S. Nat. Mus., 1898 (1900), p. 782, fig. 169.

TYPE LOCALITY: Pennsylvania and New Jersey are mentioned as habitat.

RANGE : Southern Canada through the Eastern and Middle states South to Florida and Texas, New Mexico.

\title{
Opheodrys ( $G E N U S$ )
}

Fitzinger, Syst. Rept., 1843, p. 26

TYPE: aestivus

Opheodrys aestivus (LINNÉ)

ROUGH GREENSNAKE

Coluber aestivus LinNé

Syst. Nat., Ed. 12, Vol. 1, 1766, p. 387.

Opheodrys aestivus Cope

Proc. Ac. Nat. Sci. Phila., 1860, p. 560.

Cyclophis aestivus Cope

Ann. Rep. U. S. Nat. Mus. 1898 (1900), p. 784, fig. 170.

TYPE LOCALITY: Carolina.

RANGE: New Jersey to Florida, westward to New Mexico, in the

Mississippi drainage basin found in the southern portion and northward to Kansas and Illinois.

\author{
Coluber ${ }^{1}$ ( $\left.G E N U S\right)$ \\ Linsé, Syst. Nat., Ed. 10, Vol. 1, 1758, p. 216 \\ TYPE: constrictor
}

Coluber anthonyi (STEJNEGER)

Bascanion anthonyi Stejneger

Proc. U. S. Nat. Mus., Vol. 23, June 5, 1901, p. 715.

TYPE LOCALITY : Clarion Island, Revilla Gigedo group, off west coast of Mexico.

RA NGE: Clarion Island, Revilla Gigedo group, off west coast of Mexico.

\section{Coluber aurigulus (COPE)}

Drymobius aurigulus Cope

Proc. Ac. Nat. Sci. Phila., 1861, p. 301.

1 As Coluber constrictor Linné of 1758 is composite and includes in part the black snake, Fitzinger's designation of the type of Coluber as $C$. constrictor seems to hold, as it is (partly) included in the original Coluber. 
Cohube harbouri Vautsuburgh . Sterim. Coluber hacboni Vau Duebursh r Slevin Proc. Calif. Ocad, Sei., Ser. 4, Vol. 11, July 30, 1921, p. 98. Type Pocality: bla Partida, Epiritu Santo Sland Jorrer Calif.

Rauge: Sacuc. 


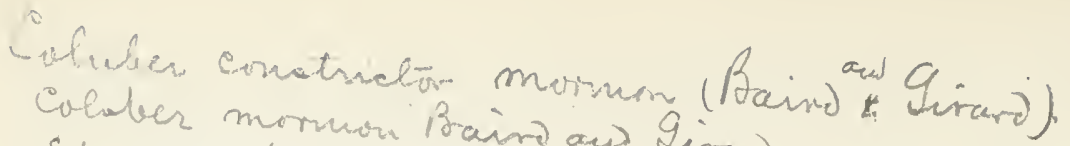
cuebler momuon Bain aw Givan

Stanchung's Ex/.. Ireat Sae* Laxe, Apsend. C, Rep\%, $1852,0,351$.

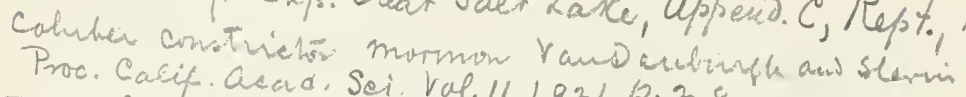

Proc. Casif. Cacad. Sei. Vap.11, $1921,10.28$

Tyle Proality: ralley of the Gues soet hate, wah.

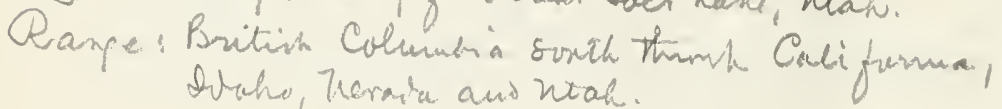
beho, Terate and noak. 


\section{Zamenis aurigulus COPE}

Ann. Rep. U. S. Nat. Mus. 1898 (1900), p. 810, fig. 179.

TyPE locality: Cape St. Lucas, Lower California.

RANGE: Southern Lower California.

\section{Coluber constrictor constrictor (LiNNÉ)}

BLACKSNAKE

Coluber constrictor (part) ' Linvé

Syst. Nat. Ed. 10, Vol. 1, 1758, p. 216.

Zamenis constrictor (part) COPE

Ann. Rep. U. S. Nat. Mus. 1898 (1900), p. 791, fig. 171.

TYPE LOCALITY : "Habitat in America septentrionale."

RANGE: The whole of the Eastern United States westward to

Texas and the Great Plains.

Coluber constrictor flaviventris ${ }^{2}\left(\mathrm{SAY}_{\mathrm{A}}\right)$

WESTERN OR BLLE RACER

Coluber flaviventris $\mathrm{S}_{A Y}$

Long”s Exp. Rocky Mts., Vol. 2. 1823. p. 185.

Coluber constrictor var. flariventris GARMAN

Mem. Mus. Comp. Zoöl., Vol. 8, Pt. 3, 1883, p. $1+7$.

TYPE LOCALITY: Stone quarry on west side of Missouri River

3 miles above the mouth of Boyer's River.

RANGE: Central, Southwestern and Paeife Coast states.

Coluber flagellum fiagellum (SHAw)

COACH WHIP

Coluber flagellum SHAW

Gen. Zool., Vol. 3, 1802, p. 475.

Zamenis flagellum flagellum COPE

Ann. Rep. Nat. Mus. 1898 (1900), p. 799 , fig. 17 .

TYPE LOCALITY: Carolina and Virginia.

RANGE: Virginia to Florida, westward to the Rocky Mountains.

\section{Coluber flagellum piceus ${ }^{3}$ (COPE)}

Bascanium piceum COPE

Proc. U. S. Nat. Mus., Vol. 14, March 28, 1892, p. 625.

Zamenis flagellum piceus CoPE

Ann. Rep. U. S. Nat. Mus. 1898 (1900), p. S04, fig. 175.

1 For remarks upon the use by Linné of the name eonstrictor see the note under Heterodon contortrix.

2 There are many colour phases within this species more or less intermediate between the black eastern form and the light coloured western form. Several of these have been given names but as yet it is not evident that they really constitute true gcographic races and hence they are not recognized at present.

3 Bascanion flogellum frenatus Stejneger 1893, not Coluber frenatus of Gravenhorst, 1807. 
TYPE LOCAlity: Camp Grant, Arizona.

RANGE: Southern Arizona.

\section{Coluber lateralis (HALlowell)}

Leptophis lateralis HaLlowell Proc. Ac. Nat. Sci. Phila., Vol. 6, 1853, p. 237.

Zamenis lateralis COPE

Ann. Rep. U. S. Nat. Mus. 1898 (1900), p. 807, fig. $17 \%$.

TYPE LOCALITY: California.

RANGE: Southern and Lower California.

Coluber schotti (BAIrd and Girard)

Masticophis schotti BAIRD and GIRARD

Cat. N. Amer. Rept., Pt. 1, 1853, p. 160.

Zamenis schotti COPE

Ann. Rep. U. S. Nat. Mus. 1898 (1900), p. 811, fig. 18a.

ty pe locality: Eagle Pass, Texas.

RANGE: Southern Texas and northeastern Mexico.

\section{Coluber semilineatus (COPE)}

Bascanium semilineatum COPE

Proc. U. S. Nat. Mus., Vol. 14, March 98, 1892, p. 626.

Zamenis semilineatus COPE

Ann. Rep. U. S. Nat. Mus. 1898 (1900), p. 805, fig. 176.

TyPe locality : Camp Grant and Colorado River, Arizona. RANGE: Arizona and the adjacent territory of Mexico.

Coluber taeniatus taeniatus (HALLOWELL)

Leptophis taeniata HaLlowelL

Proc. Ac. Nat. Sci. Phila., Vol. 6, 1852, p. 181.

Coluber taeniatus Garman

Mem. Mus. Comp. Zoöl., Vol. 8, Pt. 3, 1883, p. 46.

Zamenis taeniatus COPE

Ann. Rep. U. S. Nat. Mus. 1898 (1900), p. 815, fig. 182.

TyPE LOCAlity: New Mexico west of Rio Grande.

RANGE: Idaho and Utah, southward to all the border states just

entering Texas and California.

Coluber taeniatus girardi, nom. nov.

Masticophis ornatus ${ }^{1}$ BAIRD and GIRARD

Cat. N. Amer. Rept., Pt. 1, 1853, p. 102.

Zamenis ornatus CoPE

Ann. Rep. U. S. Nat. Mus. 1898 (1900), p. 813, fig. 181.

TYPE LOCALITY: “Between Indianola and El Paso," Texas. RANGE: Western Texas. 




\section{Salvadora (GEYYSS)}

Baird and Girard, Cat. N. Amer. Rept., Pt. 1, 1853, p. 104 TYPE: grahamiae.

Salvadora grahamiae BAIRD and (imiRI) PATCH-NOSE SNAKE

Salvadora grahamiae BARD and GIRARD

Cat. N. Amer. Pept., Pt. 1, 1S53, p. 104.

Saliadora grahamiae (part) Cope

Ann. Rep. U. S. Nat. Mus. 1898 (1900), p. 818.

TYPE LOCALITY: Sonora, Mexico.

RANGE: Utah southward through all the border states, eastward of Arizona and into Mexico as far south as the state of Hidalgo.

\section{Salvadora hexalepis (Cope)}

Phimothyra hexalepis Cope

Proc. Ac. Nat. Sci. Phila., 1866, p. 30\%.

Saliadora hexalepis Stejneger

Proc. U. S. Nat. Mus., Vol. 25, Scpt. 8. 1902, p. 154.

Salvadora grahamiae (part) CoPE

Ann. Rep. U. S. Nat. Mus. 1898 (1900), p. 818, fig. 183.

TrPe LoCALItr : Fort Whipple, Arizona.

RANGE : Arizona, California and Lower California.

RA NGE Arizona, California and Lower California.

\section{Phyilorhynchus ( $G E N U S)$}

Stejneger, Proc. U. S. Nat. Mus., Vol. 13, Sept. 9, 1S90, p. 151 TY РЕ: browni

\section{Phyllorhynchis browni STEJNEGER}

Phyllorhynchus browni StejNeger

Proc. U. S. Nat. MIus., Vol. 13, Sept. 9, 1890, p. 159.

Phyllorhynchus browni COPE

Ann. Rep. U. S. Nat. Mus. 1893 (1900), p. 891, fig. 184.

TYPE LOCALITT: Tucson, Arizona.

RANGE: Southern Arizona.

\section{Phyllorhynchus decurtatus (COPE)}

Phimothyra decurtata Cope

Proc. Ac. Nat. Sci. Phila., 1868, p. 310.

Phyllorhynchus decurtatus STEJNEGer

Proc. U. S. Nat. Mus., Vol. 13, 1890, ') 15 t.

Phyllorhynchus decurtatus Cope

Ann. Pep. U. S. Nat. Mus. 1898 (1909), p. 823, fig. 186.

TYPE LOCALITY: “In the upper part of Lower California."

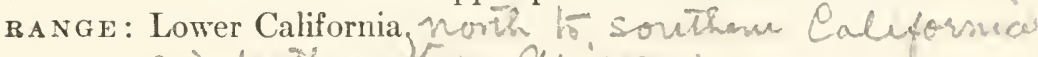
ani south westens Chizena. 


\section{Elaphe ( $G E N U S)$}

Fitzinger in Wagler, Descr. Icon. Amphib., Pt. 3, 1833, text to plate 97 TYPE: parreysii $=$ quatuorlineata

Elaphe bairdi (YaRRow)

Coluber bairdi $\mathrm{Y}_{\mathrm{ARR}} \mathrm{W}$

Bull. U. S. Nat. Mus., No. 17, 1880, p. 41.

Coluber bairdii COPE

Ann. Rep. U.S. Nat. Mus. 1898 (1900), p. 854, fig. 198.

type LOCAlity: Fort Davis, 50 miles northwest of Presidio, Texas.

RANGE: Known from type locality only.

\section{Elaphe chlorosoma (GüNTHER)}

Coluber chlorosoma GüNTHER

Biol. Centr. Amer., Rept., 1894, p. 115, pl. 41.

TyPE Locality: Atoyak and Amula in Guerrero and San Ramon in Jalisco, Mexico.

RANGE: Guerrero and Jalisco Mexico, northward to the Santa Rita Mountains in Arizona.

\section{Elaphe guttata (LrNNÉ)}

CORN-SNAKE

Coluber guttatus Linné

Syst. Nat., Ed. 12, Vol. 1, 1766, p. 385.

Elaphis guttatus DumériL and Bibron

Erp. Gén., Vol. 7. 1854, p. 273.

Coluber guttatus COPE

Ann. Rep. U. S. Nat. Mus. 1898 (1900), p. 833, figs. 189, 190. TYPE LOCALITY: Carolina.

RA NGE: Maryland to Florida, Louisiana and Mississippi.

Elaphe laeta (BAIrd and Girard)

Scotophis laetus BAIRD and GIRARD

Cat. N. Amer. Rept., Pt. 1, 1853, p. 77.

Coluber laetus COPE

Proc. U. S. Nat. Mus., Vol. 14, 1892, p. 606.

Coluber laetus COPE

Ann. Rep. U. S. Nat. Mus. 1898 (1900), p. 850, fig. 196.

Coluber emoryi COPE

Ann. Rep. U. S. Nat. Mus. 1898 (1900), p. 852, fig. 197.

TYPE LOCALITY: Red River, Arkansas.

RANGE: Kansas southward to Central Mexico. 


Elaphe obsoleta obsoleta (S.AY)

PILOT BLACKSNAKE

Coluber obsoletus $\mathrm{S}_{\mathrm{AY}}$

Long`s Exp. Rocky Mts., Vol. 1, 1823, p. 140.

Elaphis obsoletus Garman

Mem. Mus. Comp. Zoöl., Vol. 8, Pt. 3, 1883, p. 54.

Coluber obsoletus obsoletus (part) CopF

Ann. Rep. U. S. Nat. Mus. 1898 (1900), p. S4t, fig. 194.

TYPE LOCALITY: Isle au Vache [sic] to Council Bluffs on the Missouri River.

RANGE: Southern New England westward to Michigan, southward to Texas, Louisiana and Florida.

Elaphe obsoleta confinis (BAIrd and Girard)

Scolophis confinis BAIRD and GIRARD

Cat. N. Amer. Rept., Pt. 1, 1853, p. 76.

Coluber confinis Copr.

Amn. Rep. U. S. Nat. Mus. 1898 (1900), p. 829, fig. 187.

type locality: Anderson, South Carolina.

RANGE: South Atlantic and Gulf states and states of southern

Mississippi Valley.

Elaphe obsoleta lindheimerii (BAIrD and Girand)

Scotophis lindheimerii BAIRD and Girard

Cat. N. Amer. Rept., Pt. 1, 1853, p. 74.

Elaphis obsoletus var. lindheimerii Garman

Mem. Mus. Comp. Zoöl., Vol. 8, Pt. 3, 1883, p. 54.

Coluber obsoletus (part) CopE

Ann. Rep. U. S. Nat. Mus. 1898 (1900), p. 844.

Ty PE LOCALITY: New Braunfels, Texas.

RANGE: Texas.

\section{Elaphe quadrivittata (НоцвRоок)}

\section{CHICKEN-SNAKE}

Coluber quadrivittatus HoLBRoок

N. Amer. Herp., Ed. 1, Vol. 1, 1836, p. 113, pl. 21.

Elaphis quadrivittatus Dưnéril and BIBron

Erp. Gén., Vol. 7, 1854, p. 265.

Coluber quadrivittatus COPE

Amu. Rep. U. S. Nat. Mus. 1898 (1900), p. 838, fig. 192.

TYPE LOCALITY: “ North Carolina to Florida and westward as

far as the Mississippi."”

RANGE: Carolina to Florida and west to the Mississippi River. 
Elaphe rosaliae (MocQUARD)

Coluber rosaliae Moceuard

Nouv. Arch. Mus. Hist. Nat. Paris, Ser. 4, Vol. 1, p. 321, pl. 12, figs. $1-1 \mathrm{~B}$.

TYPE LOCALity : Sta. Rosalia, Lower California.

RANGE: Sta.-Rosalia, Lower California.

Cerctral mas sow thens

Elaphe subocularis (BRowN)

Coluber subocularis Brown

Proc. Ac. Nat. Sci. Phila., 1901, p. 499, pl. 29.

TyPe LOCAlity: Davis Mountains, Jeff Davis Co., 50 miles south of Pecos, Texas.

RANGE: Davis Mountains, Texas.

Elaphe vulpina (BAIRD and Girard)

FOX-SNAKE

Scotophis vulpinus BaIRD and GirarD

Cat. N. Amer. Rept., Pt. 1, 1853, p. 75.

Elaphe vulpinus RUTHVEN

Eleventh Ann. Rep. Michigan Ac. Sci., 1909, p. 110.

Coluber vulpinus COPE

Ann. Rep. U. S. Nat. Mus. 1898 (1900), p. 831, fig. 188.

TYPE LOCALITY: Racine, Wisconsin.

RANGE: Indiana, Iowa, and Illinois, to Michigan and Minnesota,

Drymobius ( $G E N U S)$

Fitzinger, Syst. Rept., 1843, p. 96

TYPE: margaritiferus

\section{Drymobius margaritiferus (ScHleget)}

Herpetodryas margaritiferus SCHLEGEL

Essai Physion. Serp., Vol. 2, 1837, p. 184.

Drymobius margaritiferus COPL

Proc. Ac. Nat. Sci. Phila., 1860, p. 561.

TYPE L OCALITy: New Orleans (in errore).

RANGE: Southwestern Texas to Venezuela and Colombia.

Drymarchon ( $G E N U S$ )

Fitzinger, Syst. Rept., 1843, p. 26

TYPE: corais

Drymarchon corais couperi (HoLeRook)

GOPHER-SNAKE, INDIGO-SNAKE

Coluber couperi HoLBrook

N. Amer. Herp., Ed. 2, Vol. 3, 1842, p. 75, pl. 16. 
$\checkmark$ Elaphe rosucea (Cope).

Coluber rosaceus Cope

Prac W. S. hat Mus., $11,1 \&\left\{8,1,3 c, 1=6, f^{2} \cdot 3\right.$

Elaphe rosace Harizin

Copcia, ho.84, July 31,192c,p.68.

Type Pocality: Key Veat, 7londa.

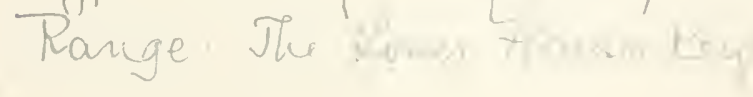


Drymarchon corais couperi STRECKER

Baylor Bulletin, Vol. 18, No. 4, Aug., 1915, p. 32.

Compsosoma corais couperii (part) COPE

Ann. Rep. U. S. Nat. Mus. 1898 (1900), p. 858, figs. 199, 200.

TYPE LOCALITY: Dry pine hills south of Alatamaha, Georgia.

RANGE: Carolinas to Florida, westward to Texas.

\title{
Drymarchon corais melanurus (Duxénil and Bibron)
}

Spilotes melanurus Duméril and Bibron

Erp. Gén.. Vol. 7, 1854, p. 22\&.

Drymarchon corais mclanurus STEJNEGER

N. Amer. Fauna, No. 14, 1899, p. 70.

TyPe LOCAlity: Mexico.

RANGE: Extreme southeastern Texas to northwestern South America.

\section{Arizona ( $G$ EN US $)$}

Kenvicott, U.S. Mex. Bound. Surv., Vol. 2, 1859, Rept. p. 18 TYPE: elegans

Arizona elegans KenNicotT

Arizona elegans KenxicotT

U. S. Mex. Bound. Surv., Yol. \&, 1859, Rept. p. 18, pl. 13.

Rhinechis elegans Cope

Ann. Rep. U. S. Nat. Mus. 1898 (1900), p. 863, fig. 201.

TYPE LOCALITY: Rio Grande, Texas, and between Arkansas and

Cimarron, Oklahoma.

RANGE: Border states of United States and northern states of Mexico.

\author{
Pituophis (GE.VUS) \\ Holbrook, N. Amer. Ferp., Ed. 2, Yol. 4, 1842, p. 7 \\ T Y PE: melanoleucus
}

Pituophis catenifer catenifer (BLAINTILLE)

WESTERN BLLL-SNAKE

Coluber eatenifer BLAINVILle

Nouv. Ann. Mus. Hist. Nat. Paris, Vol. 4, 1835, p. 290, pl. 26, figs. 9-9B.

Pituophis catenifer BAIRD and GIRARD

Cat. N. Amer. Rept., Pt. 1, 1853, p. 69.

Pityophis catenifer COPE

Ann. Rep. U. S. Nat. Mus. 1898 (1900), p. 876, fig. 208.

TYPE LOCALITY: California.

RANGE: California to British Columbia. 
Pituophis catenifer deserticola Stejneger

Pituophis catenifer desertieola STEJNeger

N. Amer. Fauna, No. 7, May 31, 1893, p. 206.

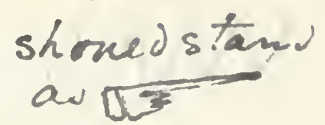

TYPE LOCALITY: Great basin and southwestern deserts.

RANGE: Desert regions of southern Californian, Nevada, and

perhaps-also southern-Idaho-and eastern Oregon.

Pituophis melanoleucus (DaUdiN)

PINE SNAKE

Coluber mclanolencus DaUdiN

Hist. Nat. Rept., Vol. 6, 1803, p. 409.

Pituophis melanoleueus НоцвRоок

N. Amer. Herp., Ed. 2, Vol. 4, 1842, p. 7, pl. 1.

Pityophis melanoleucus Cope

Ann. Rep. U. S. Nat. Mus. 1898 (1900), p. 867, fig. 203.

TYPE LOCALITY: South Carolina and Florida.
RANGE: Pine barrens of southern New Jersey to

Pituophis sayi (Schlegel)

BULL-SNAKE

Coluber sayi Schleget

Essai Physion. Serp., Vol. 2, 1837, p. 157.

Pityophis sayi sayi Cope

Ann. Rep. U. S. Nat. Mus. 1898 (1900), p. 870, fig. 204.

TYPE LOCALITY: Missouri.

RANGE: 'Texas to Minnesota.

Pituophis vertebralis (BLAinville)

Coluber vertebralis BLAinville

Nouv. Ann. Mus. Hist. Nat. Paris, Vol. 4, 1835, p. 293, pl. 27, figs. Q-21.

Pituophis vertebralis DumérIL and BIBRoN

Erp. Gén., Vol. 7, 1854, p. 238.

Pityophis vertebralis COPE

Ann. Rep. U. S. Nat. Mus. 1898 (1900), p. 879, fig. 209.

TYPE LOCALITY: California, a term which formerly included

Lower California.

RANGE: Lower California. Serwen and enitral

\section{Leimadophis $(G E N U S)$}

Fitzinger, Syst. Rept., 1843, p. 26

Dremives

$T Y P E:$ almadensis $=$ reginae

Leimadophis flavilatus (COPE)

Dromicus flavilatus CoPE

Proc. Ac. Nat. Sci. Phila., 1871, p. 222. 
Pitccaphis catenifer annectens (Baind - Irian)). Pitüophis annectens Baino + Sirind, Catt. H. Ances. Rept. Pt.1.1853,p.72.

Pituophis catenifer annecters Yant euburgh:

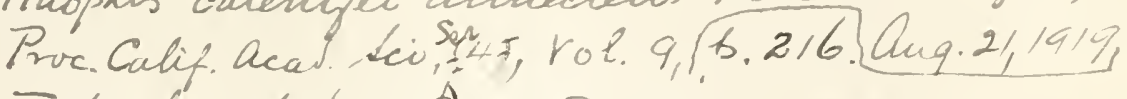
Type focality: Sun Diego, Saizínria Range: Coast Resion of Sorithere aces Notheru Pines

$\checkmark$ Pitrophis catenifer rutilus Vau Denbursh.

Pituophis catenifer rutilus Vau Denburgh. pl.2, fy.2. Pruc. Calif. Gead. Sei. Ser.4., Yol.10, Gunq.6,1920,p.24, n Type locatity: Inseon, arizona.

Range: Sornthere and ceutral Qrigonas to L. Chih.

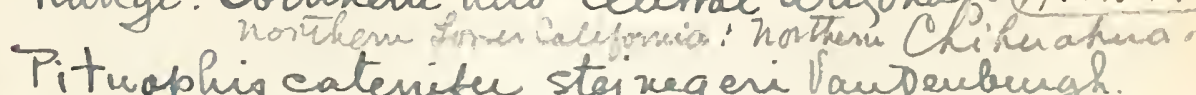
Pituophis catenefer stey regeri Vanderbengh.

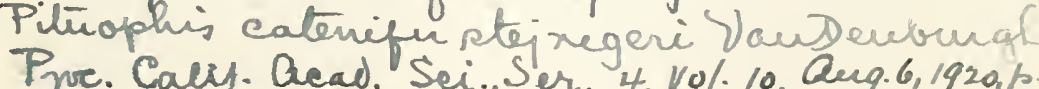

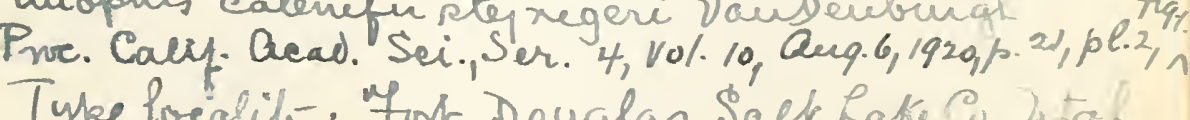
pre locialif: Fort Douglas, Salt Rake Cu. Lta h. Range: Wtah.

Pituophis eatenifer heermanni (Hallowsh) Pityophisheermami Hallowell Prove Gead. Nat. Sci. Phila, Vol. 6,1853,p. 236. Pitroghis catenifes Reermani VanDenbursh. Pre. Calip acad. Sci. Ses. 4, Vol. 10, Aug. 6,1920, p.16,pl. tiq. t.

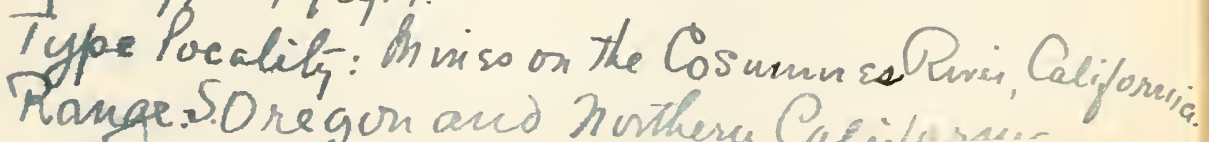

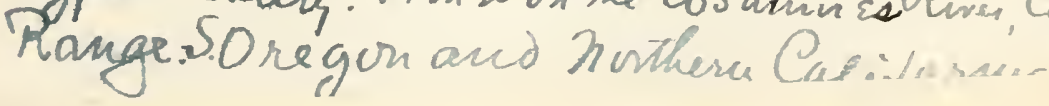

Dromicus (Yenus

Bibrms in Sa, ras Hior. Fis. Pol. Nat. Cuba, 4, 1843, 133

Type: cursor. 
Pituophis catenifer deserticola Stejneger

Pituophis catenifer deserticola STEJNeger

N. Amer. Fauna, No. 7, May 31, 1893, p. 206.

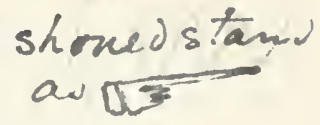

TYPE LOCALITY: Great basin and southwestern deserts.

RANGE: Desert regions of southern Californian, Nevada, and perhaps also - southern Idaho-and-eastern Oregon.

melianoleseno

Pituophis melanoleucus (DaUdiN) - 2.1\%

PINE SNAĥt

Coluber melanoleueus DAUDiN

Hist. Nat. Rept., Vol. 6, 1803, p. 409.

Pituophis melanoleueus Ноцвrook

N. Amer. Herp., Ed. Q, Vol. 4, 1842, p. 7, pl. 1.

Pityophis melanolencus Cope

Ann. Rep. U. S. Nat. Mus. 1898 (1900), p. 867, fig. 203.

TYPE LOCALITY: South Carolina and Florida. fo. Cacolina

RANGE: Pine barrens of southern New Jersey to

Pituophis sayi (Schleger)

\section{BULL-SNAKE}

Coluber sayi SCHLEgel

Essai Physion. Serp., Vol. 2, 1837, p. 157.

Pityophis sayi sayi CoPE

Ann. Rep. U. S. Nat. Mus. 1898 (1900), p. 870, fig. 204.

TYPE LOCALITY: Missouri.

RANGE: Texas to Minnesota.

Pituophis vertebralis (BLAINville)

Coluber vertebralis Blainvilue

Nouv. Amn. Mus. Hist. Nat. Paris, Vol. 4, 1835, p. 293, pl. 27, figs.

2-2в.

Pituophis vertebralis DUMÉRIL and BiBron

Erp. Gén., Vol. 7, 1854, p. 238.

Pityophis vertebralis CoPE

Ann. Rep. U. S. Nat. Mus. 1898 (1900), p. 879, fig. 209.

TYPE LOCALITY: California, a term which formerly included

Lower California.

RANGE : Lower California. Sontherw and entrob 
I

Pitccaphis cateniger amsectens (Baind - Arian)). Pituophis annectens Bains o Sirand, Catt H. Anes. Rept. Pt.1,1853,p. 72

Pituophis catenifer annecters Yan Teuburgh. Proc. Calif. Acal Leis, s, rol. 9,8, 216. Aug. 2,1919, Type focality: Sun Diego, salifoinia

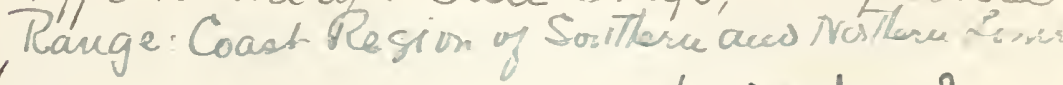

$\sqrt{ }$ Pitrophis cateniter rutilus Vau Denburss.

Pitrephis catenifer rutilus Vour Denburgh. p1.2, fip.2. Proc. Calif Gead. Sei Ses.4., Yol.10, Gung. 6, 1920,p.24, n Type Pocatity: Inseon, arizona.

Range: Sorwhere and ceutral Qrigonas to h. Chin.

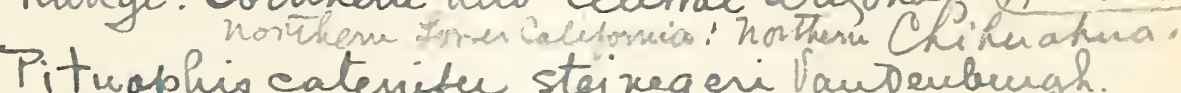
Piluophis caterifer stäreg eri Vauderenugh.

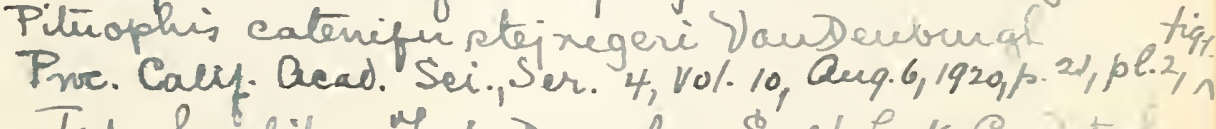
Type lociality: Fort Douglas, Salr Rake is to L. Range: Wtah.

Pituophis catenifer heermanni (Hallowsh) Pityophis heermami Hallovell Prov. Gead. Nat. Sci. Phila, Vol. 6, 1853,p. 236. Pituophis catenifer Reermani VanDenbursh. Prer. Calip acad. Sci. Sen. 4, 101.10, Ouq. 6,1920, p.16,pl. tig. 1 .

Type Pocality: On viso on the Cosumn es Rursi, Califores; Range.S.Oregon and Nothere Calijuinia

$\checkmark$ Pituophis melanolencus mugitus Barbon Piluophis melanolencus mugitus Barbon, Proc. Kew ing. Zool. Club, rol. 7, 10. 22,1921, p.11\%. Typelocality: 10 miles Hr of trest Palu Beach, Horida. Range: Peninsular Fonita. 
Lampropeltic catalin ensis raudenbugh o Slerm. Lampropelter' catalin ensis raudeubungh o Slevin.

Prviticad. Sci. Ser. 4, rol.11, Dec. 17.1921,13.397.

Type lvenlit: Sauta Calalina doland, Gref of Califorrio.

Rame: same. 
Rhadinaea flavilata Cope

Ann. Rep. U. S. Nat. Mus. 1898 (1900), p. 759, fig. 164.

TYPE LOCALITY: Fort Macon, North Carolina.

RANGE: The Carolinas to Florida.

\section{Lampropeltis ( $G E N U S)$}

Fitzinger, Syst. Rept., 1843, p. 25

TYPE: getulus Schlegel, nec Linné = holbrooki

\section{Lampropeltis alterna (BRown)}

Ophibolus alternus Brown

P'roc. Ac. Nat. Sci. Phila. 1901 (Fel., 6, 1902), p. 612.

TYPE LOCAlity: Davis Mountains, Jeff Davis Co., Texas. RANGE: Davis Mountains, Texas.

Lampropeltis boylii boylii (BAIRD and Girard)

Ophibolus boylii $\mathrm{BAIRD}$ and GIRARD

Cat. N. Amer. Rept., Pt. 1, 1853, p. 82.

Lampropeltis boylii GopE

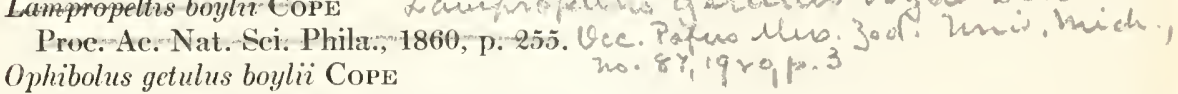

Ann. Rep. U. S. Nat. Mus. 1898 (1900), p. 919, fig. 230.

TYPE locality : El Dorado County, California.

RANGE : Arizona, western Nevada, California.

Lampropeltis boylii conjuncta CopE

Lampropeltis boylii var. conjuncta COPE

Proc. Ac. Nat. Sci., 1861, p. 301.

TYPE LOCALITY: Cape St. Lucas, Lower California.

RANGE : Southwestern Arizona and Lower California.

cali hisucial.

Lampropeltis californiae (BLAINVILLE)

Coluber (Ophis) californiae BuAinville

Nouv. Ann. Mus. Hist. Nat. Paris, Vol. 4, 1835, p. 292, pl. 27, figs.

1-1в.

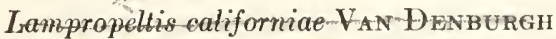

Oee. Papers Galifornia Ae. Sein, Now 5,1897, p. 172.

Ophibolus getulus califormiae COPE

Ann. Rep. U. S. Nat. Mus. 1898 (1900), p. 922, fig. 231.

TYPE LOCALITY : California.

RANGE: Southern California.

Lampropeltis calligaster ( $\left.\mathrm{S}_{\mathrm{AY}}\right)$

Coluber calligaster $\mathrm{S}_{\mathrm{AX}}$

Journ. Ac. Nat. Sci. Phila., Vol. 5, 1827, p. 359.

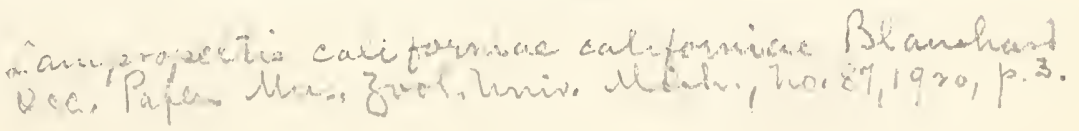


Lampropellis calligaster CoPE

Proc. Ac. Nat. Sci. Phila., 1860, p. 255.

Ophibolus calligaster COPE

Ann. Rep. U. S. Net. Mus. 1898 (1900), p. 905, fig. 223.

TYPE LOCALITY: Missouri.

RANGE: Illinois and Wisconsin to Texas.

Lampropeltis elapsoides (HoLBRoor)

Coluber elapsoides Hournook

N. Amer. Herp., Ed. 1, Vol. 2, 1838, 1. 123, pl. 28.

Osceola elapsoidea Cope

Ann. Rep. U. S. Nat. Mus. 1898 (1900), p. 900, fig. 221.

TYPE LOCALIty: South Carolina and Georgia.

RANGE: Southeastern states to Florida and Louisiana.

$$
\text { qetieluo }
$$

$\checkmark$ Lampropeltis getultis ${ }^{1}$ (LINNÉ)

KING-SNAKE, CHAN-SNAKE

Coluber getulus LiNNÉ

Syst. Nat., Ld. 19, Vol. 1, 1766, p. 882.

Lampropeltis getula COPE

Proc. Ac. Nat. Sci. Phila., 1860, p. 255.

Ophibolus gctulus gctulus COPE

Ann. Rep. U. S. Nat. Mus. 1898 (1900), p. 914, figs. $927,298$.

TYPE LOCALITY: Carolina. RANGE: Southern New England to Florida.

$$
\text { getulus }
$$

Lampropelis holbrooki STTSNEGER

Lampropeltis holbrooli STEJNEGER

Proc. U. S. Nat. Mus., Vol. 95, Sept. 8, 1902, p. 152.

Ophibolus getulus sayi $\mathrm{COPE}$

Ann. Rep. U. S. Nat. Mus. 1898 (1900), p. 911, fig. 200.

TFPE LOCALITY: Valley of the Mississippi.

RANGE: Louisiapna to Texas, northward through the states of

the Mississippi basin to-Hinois and Indiana. nues ify west of the

Lampropelís nitida VAN DENBURGH

Lampropeltis nitida $\mathrm{V}_{\mathrm{AN}}$ Densurgh mine to sowtere towe and

Proc. California Ac. Sci., Ser. 2, Vol. 5, 1895, p. 143, pl. 14. TyPE Locality: San José del Cabo, Lower California. RANGE : Southern Lower California.

Lampropeltis pyromelaéna pyromelaéna (COPE)

Ophibolus pyromclanus $\mathrm{COPE}$

Proc. Ac. Nat. Sci. Plila., 1866, p. 305.

1.The various forms elosely allied to getulus are all treated binominally since they are as yet very iwperfectly known.

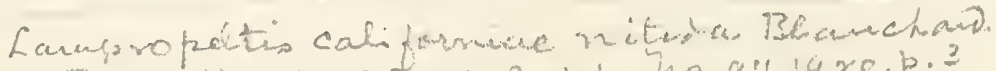

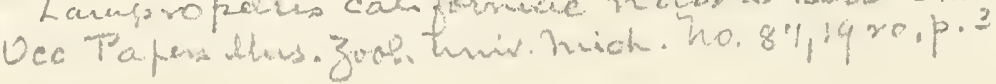


Lacupropeltis getulus yumanenis Blanchan Carrforepeltis getulus Gumanemin Bfanchas vec, Pafus Mus. 3ool. Wniv mich, ho. 81,1919,p.6,pl. 1, fis.

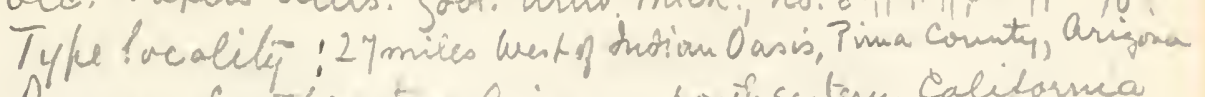
Rampe: Sonthinesterse Arizona, sowihsartan Califorma and asficent areas $Z$ Sunora and fromer Caligarma.

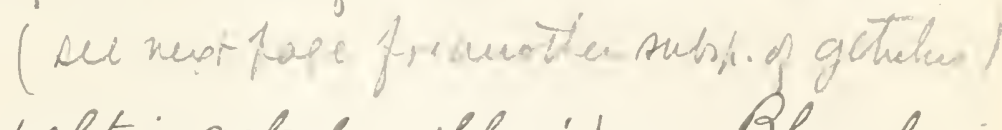
Lampropeltis qeluhs floridana Blanchar Lauepropeltis qelutus Ftoridana Blanchard, Clec. Papers Inuo. Zool. Iniv. Inich., Y0, hay 5, 1919, p. 1,p/1, Ty/e locality: Orange Hammaet, De Soto Co., Firide: Range: 7 foricla from Cranqe Ca mi the horth ló entral dade Co. ni the sonth East.

Xampropeltis qelühs hooksi Bacbour

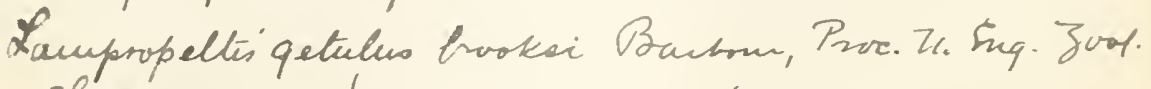
club., wol. 7, fune 5, 1919, p. 1, p1.1.

Tyle fucality: Royal Palm Hawnuoct, 14 miles Sonth vert of Atomestead Dabe C. S. E. F forida.

Range: The trofecal sonth Eastern corsue of tade Co. Florida.

$\checkmark$ Lampropeltis Elapsoides virginiana Blanchard. Lampropelliselapooides viginiana Blanchand, Occ. Pafess mus. Zuot. Univ. Inich.rosi, ape. 28,1920, p. 2

Tylue fucalily: Raleigh, North'Caroliua

Range . Nowth Carolina and Viginia, East y the alegheny mt. 
Leupowientis zenata (Lexingtón).

Bairshis zonatus dockenghion

Pro, Calif. Daa d. Sei. Yot. 7, 1877/0.52.

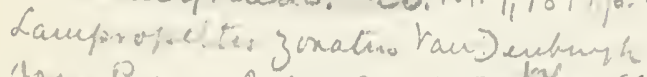

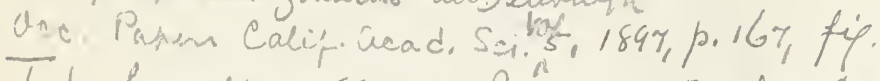

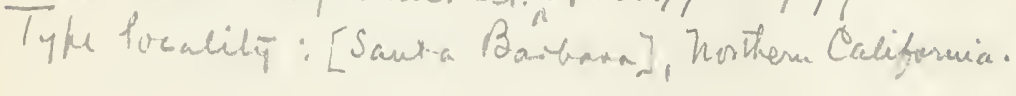

7 Lasupereet tij getuhes nigra (Yarrow) uphibokn getrulus nies Yarnow. Pro, Z. 5. hat. Mus, Vor. 5, p. 438

Lamporitaitio getelus nipes Blanchad

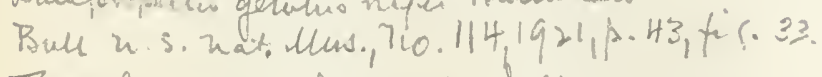

Type localiti: Wheattand, dritama.

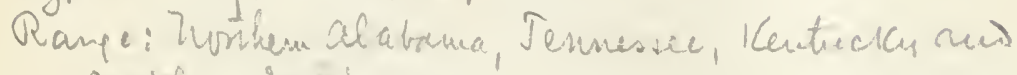
sivitiene heitioure. 
Lampropeltis pyrhomelacna STEJNEGER

Proe. U. S. Nat. Mus., Vol. 95, 1902, p. $15 \%$.

Ophibolus pyrrhomelas COPE

Ann. Rep. U. S. Nat. Mus. 1898 (1900), p. 907. fig. gat.

TYPE LoCALity: Fort Whipple, Arizona.

RANGE: Southern Arizona.

Lampropeltis pyromelaena celaenops STEJNEGER

Lampropeltis pyrrhomelaena celaenops STEJNEGER

Proc. U. S. Nat. Mus., Vol. 95, Sept. 8, 1909, p. 153.

Type Locality: Mesilla Valley, New Mexico.

RANGE: New Mexico and Eastern Arizona.

\section{Lampropeltis pyromelaena multicincta (YAnizow)}

Ophibolns yetulus multicinetus Yanrow

Proc. U. S. Nat. Mus., Vol. 5. 1889, p. 410.

Lampropeltis pyrthomelaena multicincta STEJNEGER

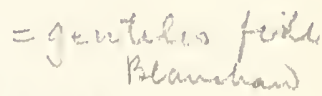

onit

$=3 v i a \hat{a}$

Proc. U. S. Nat. Mlus., Vol. 25, Sept. 8, 1902, p. 153.

TrPe LoCAlity: Fresno, California.

RANGE: California.

Lampropeitis rhombomaculata (HoLerook)

Coluber rhombomaculatus HoLBRoOK

N. Amer. Uerp., Ed. 1, Vol. 4, 1840. p. 103, pl. 20.

Lampropeltis rhombomaculata COPE

Proc. Ac. Nat. Sci. Phila., 1860, p. 955.

Ophibolus rhombomaculatus COPE

Ann. Rep. U. S. Nat. Mus. 1898 (1900), p. 803 , fig. 222.

TYPE LOCALITY: Georgia and Alabama.

RANGE: Maryland to Georgia and Alabama.

Lampropeltis splerdida (BAIRD and Cirard)

Ophibolus splendidus BAIRD and GirarD

Cat. N. Amer. Rept., Pt. 1, 1853, p. 83.

Lampropeltis splendiala COPE

Proc. Nat. Sci. Phila., 1860, p. 255.

Oplibolus yetulus splcndidus COPE

Ann. Rep. U. S. Nat. Mus. 1898 (1900), p. 918, fig. 229.

TYPE LOCAlity: Sonora, Mexico.

RANGE: Northern Mexico and the Boundary region.

Lampropeltis triangulum triangulum (LACÉPÈDE)

MIILK-SNAKE

Coluber triangulum LACÉPÈDE

Hist. Nat. Quadr. Ovip. Serp., Vol. \&, 1788, tahl. méth., p. 86. 
Lampropeltis triangula CopE

Proc. Ac. Nat. Sci. Phila., 1860, p. 256.

Osceola doliata triangula COPE

Ann. Rep. U. S. Nat. Mus. 1898 (1900), p. 885, figs. 210-215.

TYPE LOCALITY : America.

RANGE: Eastern North America from Massachusetts to Iowa, south to Virginia and in the mountains further south.

\section{Lampropeltis triangulum amaura (CopE)}

Lampropeltis amaura CopE

Proc. Ac. Nat. Sci. Phila., 1860, p. 258.

Osceola doliata coccinea Cope

Ann. Rep. U. S. Nat. Mus. 1898 (1900), p. 896, figs. 220, 216, (not of Schlegel).

TYPE LOCALITY : “Unknown" (original entry of type U.S. Nat. Mus. No. 5282 reads " ? Mississippi ").

RANGE: Eastern United States, west to Texas, south to the Gulf.

Lampropeltis triangulum gentilis (BAIRD and GIRARD)

Ophibolus gentilis BA1RD and Girard

Cat. N. Amer. Rept., Pt. 1, 1853, p. 90.

Osceola doliata gentilis COPE

Ann. Rep. U. S. Nat. Mus. 1898 (1900), p. 894, figs. 218, 219.

TYPE LOCALITY: North Fork, Red River, near Sweetwater

Creek, Wheeler Co., Texas.

RANGE: Texas and northwestern Mexico.

\section{Stiloma}

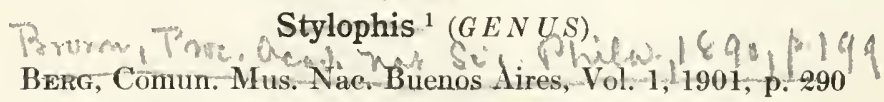

\section{Stilionmos} TYPE: extenuatusm

\section{Stylophis extenuatus (Brown)}

Stilosoma extenuatum Brown

Proc. Ac. Nat. Sci. Phila., 1890, p. 199.

Stilosoma extenuatum CopE

Ann. Rep. U. S. Nat. Mus. 1898 (1900), p. 924, fig. 232.

TYPE LOCALITY : Lake Kerr, Marion County, Florida.

RANGE : North central Florida. 
Extiahiuitäe Intered by earor mit.

Lnmpropellis triaraulum nelsmi Blarchard Lampropelts triangúlum nelsoni Slanch ari, Occ.

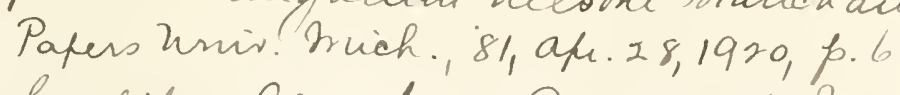

Type lucality: Acaubaro, Guana fuato, inex.

Lampropeltis tianqulem anmeato (Kenmenit)

Lanpropeltio armilata Kerrricutt

Proc acad. hat. Sci. Philo., $860,0.329$

Lampopectis triaugulum a inniesta Blandead

Bues.u.s. 2at. Mnus., 114,19=1,p 159.

Tylu prealif;: Matarnoros, mevico.

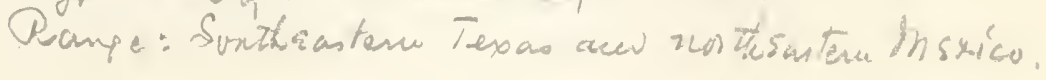

Qumpropeltis trianyulem syopila (Coss). Aphibolus daliaters syspilus cope Proc.u. s. Wat. mus., rof. 11 , p. 384.

Fanparepultio triany uhm oyspila Ruchrem

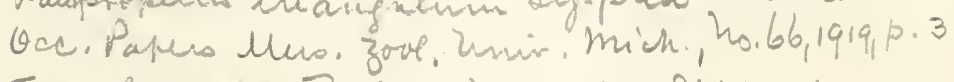
Typle ivalike: Richland Conutä, Rlinoie.

Rayge: Aricansew and Tesmessee nontwars to Dorra, Alcinois cues Anviana. 

Contia (GENUS)

Baird and Girthd, Cat. N. Amer. Rept., Pt. 1, 1853, p. 110 TYPE: mitis $=$ tenuis

Contia tenuis (BAIrD and Grrard)

Calamaria tenuis BAIRD and GIRARD

Proc. Ac. Nat. Sci. Phila., Vol. 6, 1859, p. 176.

Contia mitis CoPE

Ann. Rep. U. S. Nat. Mus. 1898 (1900), p. 925, fig. 233.

Lodia temis Cope

Ann. Rep. U.S. Nat. Mus. 1898 (1900), p. 927, fig. 234.

TYPE LOCALITY: Puget Sound, Washington.

RANGE: Southern California to Puget Sound.

\section{Cemophora (GENUS)}

Cope, Proc. Ac. Nat. Sci. Phila., 1860, p. 244

TYPE : coccinea

\section{Cemophora coccinea (Blumentach)}

Coluber coccineus Blumexbach

Voigt’s Mag. Neu. Phỵs. Naturg., Vol. 5, Pt. 1, 1788, p. 11, pl. 1.

Cemophora coccinea Cope

Proc. Ac. Nat. Sci. Phila., 1860, p. 244.

Cemophora coccinea Cope

Ann. Rep. U. S. Nat. Mus. 1898 (1900), p. 928, fig. 235.

TYPE LOCALITY: Florida.

RANGE: Maryland to Alabama, Louisiana and Florida.

\section{Rhinocheilus (GENUS)}

Baird and Girard, Cat. N. Amer. Rept., Pt. 1, 1853, p. 120 TYPE : lecontei

Rhinocheilus lecontei BAIRD and GIRARD

Rhinocheilus lecontei BAIRD and GIRARD

Cat. N. Amer. Rept., Pt. 1, p. 120.

Rhinochilus lecontei COPE

Ann. Rep. U. S. Nat. Mus. 1898 (1900), p. 931, fig. 236.

TYPE locality: San Diego, California.

RANGE: Southern Galifornia to Texas, northern Mexico.

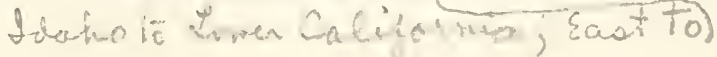




\author{
Sonora $(G E N U S)$ \\ Baird and Girard, Cat. N. Amer. Rept. Pt. 1, 1853, p. 117 \\ TYPE: semianmulata
}

\title{
Sonora episcopa (KENNICOTT)
}

Lamprosoma episcopum KENNICOTT

U. S. Mex. Bound. Surv., Vol. 2, 1859, Rept. p. 22, pl. 8, fig. 2. Sonora episcopa VAN DENBURGH

Proc. California Ac. Sci., Ser. 4, Vol. 3, 1913, p. 411.

Chionactis episcopus Cope

Ann. Rep. U. S. Nat. II 1898 (1900), p. 937, fig. 238.

type locality: Eagle Pass, and between San Antonio and the Rio Grande, Texas.

RANGE: Texas to southeastern California and the adjacent regions of Mexico.

\section{Sonora occipitalis (HOLLOWELL)}

Rhinostoma occipitale HALLOWELL

Proc. Ac. Nat. Sci. Phila., Vol. 7, 1854, p. 95.

Sonora occipitalis VAN DenbURGH

Proc. California Ac. Sci., Ser. 4, Vol. 3, 1913, p. 411.

Chionactis occipitalis COPE

Ann. Rep. U. S. Nat. Mus. 1898 (1900), p. 941, fig. 240.

TYPE LOCALITY: Mojave Desert.

RANGE : Southeastern California, Arizona, Colorado

\section{Sonora semiannulata BAIRD and Girard}

Sonora semianmulata $\mathrm{B}_{\mathrm{AIRD}}$ and GirarD

Cat. N. Amer. Pept., Pt. 1, 1853, p. 117.

Chionactis episcopus isozonus Cope

Ann. Rep. U. S. Nat. Mus. 1898 (1900), p. 939, fig. 239.

TyPE LoCALity: Sonora, Mexico.

RANGE: Utah to Nevada, Arizona, southern Kansas and Texas.

\section{Sonora taylori (Boulenger)}

Contia taylori Boulenger

Cat. Snakes Brit. Mus., Vol. \&, 189ł, p. 265, pl. 12, fig. 3.

Chionactis taylorii Cope

Ann. Rep. U. S. Nat. Mus. 1898 (1900), p. 936.

TyPe Locality: Duval County, Texas, and Nuevo Leon,

Mexico.

RANGE: Southern Texas and northeastern Mexico. 

Chilomeniscus cinatirs Cope

Chilomeniscus cinctios Cope.

Proc. Acad. hat. Sci Philas.1 1861,p.303.

Type locality: Guayman, mexico.

Range: Colorabo Desert to ady asent Sonora and Centrac fomer Califormios. 


\section{Ficimia (GENUS)}

Gray, Cat. Snakes Brit. Mus., 1849, p. 80

Ficimia cana (Cope)

TYPE: olivacea

Gyalopion canum COPE

Proc. Ac. Nat. Sei. Phila., 1860, p. 248.

Ficimia cana Garman

Mem. Mus. Comp. Zoöl., Vol. 8, P'. 3, 1883, p. 83.

Gyalopium canum COPE

Ann. Rep. U. S. Nat. Mus. 1898 (1900), p. 947, fig. 211.

TYPE loCality: Fort Buchanan, Arizona.

RANGE: Western 'Texas to Arizona.

\section{Hiypsiglena ( $G E N U S)$}

Cope, Proc. Ac. Nat. Sci. Phila., 1860, 1. 946

TYPE: ochrorhynchus

\section{Hypsiglena ochrorhynchus ochrorhynchus (Cops)}

Hypsiylena ochrorhynchus Cope

Proc. Ac. Nat. Sci. Phila., 1860, p. 246.

IIypsiglena ochrorhyncha COPE

Ann. Rep. U. S. Nat. Mus. 1898 (1900), p. 953, fig. 245.

TyPe Locality: Cape St. Lucas, Lower California.

RANGE : Southern and Lower California, southem Arizona, north-

western Mexico.

Hypsiglena ochrorhynchus texana (STEJNEGER)

IIypsiglena terana Stejneger

N. Amer. Fauna, No. 7, 1893, p. 205.

TYPE Locality: Between Laredo and Camargo, Texas.

RANGE: Southwestern Texas.

\section{Chilomeniscus ( $G E N U S)$}

Cope, Proc. Ac. Nat. Sci. Phila., 1860, p. 339

TYPE: stramineus

$\sqrt{ }$ Chilomeniscus ephippicus COPE

Chilomeniscus ephippicus COPE

Proc. Ac. Nat. Sci. Phila., 1867, p. 85.

Chilomeniscus ephippicus COPE

Ann. Rep. U. S. Nat. Mus. 1898 (1900), p. 950, fig. 243.

TyPE LOCALIty : Owens Valley, California.

RANGE: Southern Arizona and California. 


\section{Chilomeniscus fasciatus Cope)}

Chilomeniscus stramineus fasciatus COPE

Proc. U. S. Nat. Mus., Vol. 14, 1892, p. 595.

Chilomeniscus fasciatus Cope

Ann. Rep. U. S. Nat. Mus. 1898 (1900), p. 951, fig. 244.

ty pe locality: La Paz, Lower California.

RANGE : Lower California.

\section{Chilomeniscus stramineus Cope}

Chilomeniscus stramineus CoPE

Proc. Ac. Nat. Sci. Phila., 1860, p. 339.

Chilomeniscus stramineus CopE

Ann. Rep. U. S. Nat. Mus. 1898 (1900), p. 949, fig. 242.

TYPE LOCALITy: Cape St. Lucas, Lower California.

RANGE: Southern Lower California.

\section{Natrix $(G E N U S)$ \\ LaUrentr, Syn. Rept., 1768, p. 73 \\ TYPE : vulgaris $=$ natrix}

\section{Natrix clarkii (BAIRD and Girard)}

Regina clarkii BAIRD and GIRARD

Cat. N. Amer. Rept., Pt. 1, 1853, p. 48.

Natrix clarkii COPE

Proc. U. S. Nat. Mus., Vol. 14, 1892, p. 669.

Natrix clarkii COPE

Ann. Rep. U. S. Nat. Mus. 1898 (1900), p. 987, fig. 261.

TYPE LOCALITY: Indianola, Texas.

RANGE: Coastal regions of northwest Florida to Texas.

Natrix compressicauda (KENNICOTT)

SALT-WATER MOCCASIN

Nerodia compressicauda KenNicott

Proc. Ac. Nat. Sci. Phila., 1860, p. 335.

Natrix compressicauda COPE

Proc. U. S. Nat. Mus., Vol. 11, 1889, p. 392.

Natrix compressicauda CoPE

Ann. Rep. U. S. Nat. Mus. 1898 (1900), p. 978, fig. 255.

TYPE LOCALITY: Tampa Bay, Florida.

RANGE: Southwestern Florida, especially the mangrove swamps of the extreme southern coast and the Keys.

Natrix cyclopion (DumÉRIL and BIBRoN)

Tropidonotus cyclopion Duméril and Bibron

Erp. Gén., Vol. 7, 1854, p. 576. 
Chilomeniscus punctafissimus rawheubursh o blevin chilomeniscus pructatiosimus Tau deubursh s Slevin Proc. Calif. Acad. Sei. Ser.4, rot. 11, July 30, 1921,10.98 Iype Pveality: Sla Partida, Sopiritu Santo stand, Lower Calif.

Rauge: sacue. 

Natrix cyc'opium Cope

Proc. U. S. Nat. Mus., Vol. 14, 1892, p. 673.

Natrix cyclopium Cope

Ann. Rep. U. S. Nat. Mus. 1898 (1900), p. 961, figs. 247, 248.

TYPE LOCALITY: New Orleans.

RANGE: Florida to Louisiana and northward up the Mississippi

Valley to Southern Illinois.

Natrix grahamii (BAIRD and Girard)

Regina grahamii BAIRD and GiRARD

Cat. N. Amer. Rept., Pt. 1, 1853, p. t7.

Natrix grahamii COPE

Proc. U. S. Nat. Mus., Vol. 14, 1899, p. 668.

Natrix grahamii Cope

Ann. Rep. U. S. Nat. Mus. 1898 (1900), p. 991, fig. 264.

TYPE LOCALity: Rio Salado, Texas.

RANGE: Illinois southward to Louisiana and Texas.

Natrix kirtlanỏii (KENNicotT)

Regina lirtlandii Кемхісотт

Proc. Ac. Nat. Sci. Phila., 1856, p. 95.

Natrix kirtlandii Cope

Ann. Rep. U. S. Nat. Mus. 1898 (1900), p. 995, fig. 266.

TYPE LOCALITY: Northern Illinois.

RANGE: Michigan, Wisconsin, Illinois, Indiana, and Ohio.

Natrix rhombifera (HALlOwELL)

Tropidonotus rhombifer Hallowell

Proc. Ac. Nat. Sci. Phila., Vol. 6, 1859, p. 17\%.

Natrix rhombifera Cope

Proc. U. S. Nat. Mus., Vol. 11, 1889, p. 398.

Natrix rhombifera Cope

Rep. U. S. Nat. Mus. 1898 (1900), p. 963, fig. 249.

TYPE LOCALITY: Arkansas River and its tributaries near the northern boundary of the Creek Nation.

RANGE: Southern Illinois and Indiana south to Texas and

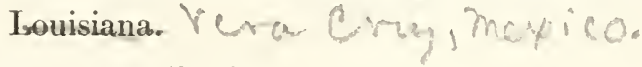

Natrix rigida ( $\mathrm{SAY}$ )

Coluber rigidus $\mathrm{S}_{\mathbf{A Y}}$

Journ. Ac. Nat. Sci. Phila., Vol. 4, Pt. 2, 1825, p. 239.

Natrix rigida Cope

Proc. U. S. Nat. Mus., Vol. 14, 1899, p. 668.

Natrix rigida Cope

Ann. Rep. U. S. Nat. Mus. 1898 (1900), p. 989, fig. 262.

TYPE LOCALITy: The Southern States.

RANGE: The Carolinas to Florida. 
Natrix septemvittata ( $\mathrm{SAY}$ )

MOON-SNAKE, QLEEN-SNAKE

Coluber septemvittatus $\mathrm{S}_{\mathrm{AY}}$

Journ. Ac. Nat. Sci. Phila., Vol. 4. Pt. 2, 18@5, p. 240.

Natrix septemvittata COPE

Trans. Amer. Philos. Soc., Vol. 18, 1895, p. 216.

Natrix leberis ${ }^{1}$ COPE

Ann. Rep. U. S. Nat. Mus. 1898 (1900), p. 993, fig. 265.

TYPE LOCALITY: Pennsylvania.

RANGE: Central, Eastern and Gulf States from Pennsylvania,

Ohio and Wisconsin southward.

\section{Natrix sipedon sipedon (LinNÉ)}

WATER SNAKE

Coluber sipedon LINNÉ

Syst. Nat., Ed. 10, Vol. 1, 1758, p. 219.

Natrix sipedon Kirsch

Bull. U. S. Fish Comm., Vol. 14, 1895, p. 333.

Natrix fasciata sipedon COPE

Anı. Rep. U. S. Nat. Mus. 1898 (1900), p. 969, fig. 251.

TYPE LOCAlity: North America.

RANGE: Northern and northeastern United States.

\section{Natrix sipecion-fasciata ${ }^{2}$ (LINNÉ)}

Coluber fasciatus Linné

Syst. Nat., Ed. 12, Vol. 1, 1766. p. 378.

Natrix fasciata fasciata CoPE

Ann. Rep. U. S. Nat. Mus. 1898 (1900), p. 966, fig. 250.

TYPE LOCALITY: Carolina.

RANGE: Virginia to Florida, westward to Louisiana and northward up the Mississippi through all the central states to Nebraska and Indiana.

\section{Natrix sipedon pictiventris CopE}

Natrix fasciata pictiventris $\mathrm{COPE}$

Amer. Nat., Vol. 29, 1895. p. $67 \%$.

Natrix fasciata pictiventris CopE

Ann. Rep. U. S. Nat. Mus. 1898 (1900), p. 969.

TYPE LOCALITY: Palatka, Florida.

RANGE: Peninsula of Florida.

1 Not Coluber leberis of Linné.

2 Apparent forms which are more or less uniformly rufeseent are found in the ranges of both the subspecies fasciaia and sipedon. To these the names of Natrix sipedon erythrogaster Forster (in Bossu, Travels through North America, Vol. 1, 1771, p. 364, footnote) and Natrix sipedon fultiventer Rafinesque (Ann. Nat., 1820, No. 1, p. 6) have been given. 


Natrix sipedon transversa (HAILOWELL)

Tropidonotus transtersus HALLOWELI,

Proc. Ac. Nat. Sci. Phila., Vol. 6, 1859, p. $17 \%$.

Natrix sipedon transcersa HURTel

Herp. Missouri. 1911, p. $15 \%$.

Natrix fasciata transversa CopE

Ann. Rep. U. S. Nat. Mus. 1898 (1900), p. 973, fig. 25 s.

TYPE LOCALITY: Creek boundary, found near the banks of the

Arkansas and its tributaries.

rANGE: Texas, Oklahoma, Arkansas.

\section{Natrix taxispilota (НоцвRоок)}

WATER-PILOT

Tropidonotus taxispilotus HoLbrook

N. Amer. Herp., Ed. 2, Vol. 4, 1842, p. 35, pl. 8.

Natrix taxispilota Cope

Proc. U. S. Nat. Mus.. Vol. 11, 1889, p. 399.

Natrix taxispilota COPE

Ann. Rep. U. S. Nat. Mus. 1898 (1900), p. 959, fig. 246.

TyPE LOCALITy: South Carolina seaboard and the Altamaha

River, Georgia.

RANGE: The Carolinas to Florida, west to Louisiana.

\section{Natrix valida (KENNICOTT)}

Regina valida KennicotT

Proc. Ac. Nat. Sci. Phila., 1860, p. 334.

Natrix valida COPE

Proc. U.S. Nat. Mus., Vol. 14, 1892, p. 670.

Natrix valida Cope

Ann. Rep. U. S. Nat. Mus. 1898 (1900), p. 984, figs. 258, 259.

ty y L L OCAlity: Durango, Mexico.

RANGE: Western Mexieo, Lower California.

\section{Seminatrix ( $G$ EN $U S)$}

Cope, Amer. Nat., Vol. 29, 1895, p. 678

TYPE: pygaea

Seminatrix pygaea (Cope)

Contia pygaea $\mathrm{Cope}$

Proc. Ac. Nat. Sci. Phila., 1871, p. 223.

Seminatrix pygaea Cope

Amer. Nat., Vol. 99, 1895, p. 678.

Seminatrix pygaea Cope

Ann. Rep. U. S. Nat. Mus. 1898 (1900), p. 998, fig. 267.

TYPE LOCAlity: Volusia, Florida.

RANGE: Florida peninsula. 


\section{Storeria $(G E N U S)$}

Baird and Girard, Cat. N. Amer. Rept., Pt. 1, 1853, p. 135 TYPE: dekayi

\section{Storeria dekayi (HoLbRook)}

\section{DEKAY'S SNAKE}

Tropidonotus deliayi HoLвroок

N. Amer. Herp., Ed. 2, Vol. 4, 1842, p. 53, pl. 14.

Storcria deliayi BAIRD and GIRARD

Cat. N. Amer. Rept., Pt. 1, 1853, p. 135.

Storeria dekayi Cope

Ann. Rep. U. S. Nat. Mus. 1898 (1900), p. 1000, fig. 268.

TY PE LOCALITY: Massachusetts and New York.

RANGE: Eastern North America, Ontario to Vera Cruz, west to Kansas.

\section{Storeria occipito-maculata (STORER)}

RED-BELLIED SNAKE

Coluber occipito-maculatus STORER

Rep. Fish. Rept. Birds Massachusetts, 1839, p. 230.

Storeria occipito-maculata BAIRD and GIRARD

Cat. N. Amer. Rept., Pt. 1, 1853, p. 137.

Storeria occipitomaculata CoPE

Ann. Rep. U. S. Nat. Mus. 1898 (1900), p. 1003, fig. 269.

TYPE LOCAlity : Amherst, Mass.

RANGE: Ontario to Vera Cruz, westward to Kansas, nomfermal

\section{Storeria victa $\mathrm{HAY}_{\mathrm{Y}}$}

Storcria victa $\mathrm{HAY}_{\mathrm{A}}$

Science ( $N$ S.), Vol. 19, April 8, 1892, p. 199.

TYPE LOCALITY: Oklawaha Rirer, some distance north of Kissimmee, Florida.

RANGE : Florida.

\section{Virginia ( $G E N U S)$}

Baird and Girard, Cat. N. Amer. Rept., Pt. 1, 1853, p. 127

TYPE: valeriae

\section{Virginia elegans ḰENNICOTT}

Virginia clegans KenNicotT

Proc. Ac. Nat. Sci. Phila, 1859, p. 99.

Virginia elegans CoPE

Ann. Rep. U. S. Nat. Mus. 1898 (1900), p. 1007, fig. 271.

TYPE LOCALITY: Heavily timbered regions of southern Illinois. RANGE: Illinois, Indiana and Missouri southward to Texas. 


Virginia valeriae BAIRD and GIRARD

Virginia valeriae BAIRD and GIRAlid

Cat. N. Amer. Rept., Pt. 1, 1853, p. 127.

Virginia valeriue CoPE

Amn. Rep. U. S. Nat. Níus. 1898 (1900), p. 1006, fig. 270.

TYPE LOCALITY : Kent County, Maryland.

RANGE: New Jersey to Soutl Carolina, west to Tennessee.

\section{Amphiardis $(G E N U S)$}

Cope, Proc. U.S. Nat. Mus., Vol. 11, 1889, p. 391

TYPE: inornata

Amphiardis inornatus (GARMAN)

Virginia inornata GARMAN

Mem. Mus. Comp. Zoöl., Vol. 8, Pt. 3, 1883, p. 97.

Amphiardis inornatus Cope

Proc. U.S. Nat. Mus., Vol. 14, 1892, p. 675.

Amphiardis inornatus COPE

Ann. Rep. U. S. Nat. Mus. 1898 (1900), p. 1009.

TyPE LOCAlity: Dallas, 'Texas.

RANGE: Texasand

\section{Potamophis (GENUS)}

Fitzinger, Syst. Rept., 1843, p. 25

TY Y E : striatulus

\section{Potamophis striatulus (LinNé)}

Coluber striatulus LinNÉ

Syst. Nat., Ed. 19. Vol. 1, 1766, p. 375.

Potamophis striatulus GARuaN

Bull. Essex Inst., Vol. 24, 1892, p. 105.

Haldea striatula COPE

Ann. Rep. U. S. Nat. Mus. 1898 (1900), p. 1009, fig. 979.

TYPE LOCALITY: Carolina.

RANGE: Virginia and Minnesota southward to the Gulf States.

\section{Tropidoclonion ( $G E N U S)$}

Cope, Proc. Ac. Nat. Sci. Plila., 1860, p. 76

TYPE: lineatum

Tropidoclonion lineatum (HALLOWELL)

Microps lineatus HaLlowell

Proc. Ac. Nat. Sci. Phila., 1856, p. 241.

Tropidoclonion lincatum Cope

Proc, Ac. Nat. Sci. Phila., 1860, p. 76. 
Tropidoclonium lineatum CoPE

Ann. Rep. U. S. Nat. Mus. 1898 (1900), p. 1011, fig. 273.

TYPE LOCALITY: Kansas.

RANGE: Ohio to Iowa and southeastern South Dakota and through Missouri, Kansas and Arkansas to Oklahoma and Texas.

\section{Liodytes ( $G E N U S)$}

Cope, Proc. Amer. Philos. Soc., Vol. $22,188 \dot{5}$, p. 194 TYPE: alleni

\section{Liodytes alleni (Garran)}

Helicops alleni Garman

Proc. Boston Soc. Nat. Hist., Vol. 17, 1874, p. 92.

Liodytes alleni Cope

Proc. U. S. Nat. Mus., Vol. 11, 1888, p. 393.

Liodytes allenii Cope

Ann. Rep. U. S. Nat. Mus. 1898 (1900), p. 1013, fig. 274.

TYPE LOCALITY: Jacksonville, Florida.

RANGE: Florida.

Thamnophis ( $G E N U S$ )

Fitzinger, Syst. Rept., 1843, p. 26

TYPE: sauritus

THE GARTER-SNAKES

Thamnophis angustirostris (KENNICOTT)

Eutaenia angustirostris KenNicotT

Proc. Ac. Nat. Sci. Phila., 1860, p. 332.

Thamnophis angustirostris RUTHVEN

Bull. U. S. Nat. Mus., No. 61, 1908, p. 120.

Eutaenia angustirostris COPE

Ann. Rep. U. S. Nat. Mus. 1898 (1900), p. 1047, fig. 290.

TYPE LOCALITY: Parras, Coahuila, Mexico.

RANGE: Southeastern Arizona and southwestern New Mexico, south to Coahuila and Durango.

\section{Thamnophis butleri (COPE)}

Eutaenia butleri Cope

Proc. U. S. Nat. Mus., Vol. 11, 1889, p. 399.

Thamnophis butleri STEJNEGER

Proc. U. S. Nat. Mus., Vol. 17, 1895, p. 593.

Eutaenia butlerii COPE

Ann. Rep. U. S. Nat. Mus. 1898 (1900), p. 1031, fig. 281.

TYPE LOCALITY: Richmond, Indiana.

RANGE: Western Pennsylvania, through Ohio and Indiana, southern Michigan. 


Thamnophis eques (REuss)

Coluber eques Reuss

Mus. Seuckenberg. Abh., Vol. 1, 1S34, p. 152, pl. 8, fig. 2.

Thamnophis eques RUTHVEN

Bull. U. S. Nat. Mus., No. 61, 1908, p. 158.

Eutaenia eques COPE

Ann. Rep. U. S. Nat. Mus. 1898 (1900), p. 1049, figs. 291-293.

TYPE LOCALITY: Mexico.

RANGE: Arizona and New Mexico southward to Guatemala.

Thamnophis hammondii (KENNICOTT)

Eutaenia hammondii KENNICOTT

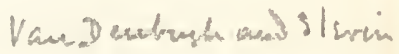

Proc. Ac. Nat. Sci. Phila., 1860, p. 322.

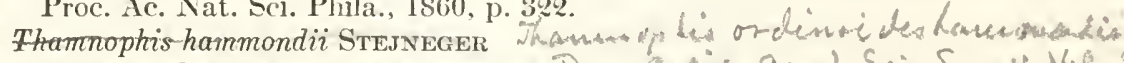

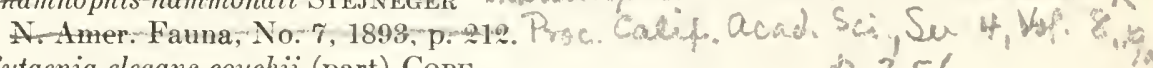

Eutaenia elegans couchii (part) COPE

Ann. Rep. U. S. Nat. Mus. 1898 (1900), p. 1019, fig. 288.

TyPE LOCALITY: San Diego and Fort Tejon, California.

RANGE: Southern California and northern Lower California.

Thamnophis marcianus (BAIRD and Girard)

Eutainia marciana BAIRD and GIRARD

Cat. N. Amer. Rept., Pt. 1, 1853, p. 36.

Thamnophis marcianus RUthveN

Bull. U. S. Nat. Mus., No. 61, 1908, p. 58.

Eutaenia elegans marciana Cope

Ann. Rep. U. S. Nat. Mus. 1898 (1900), p. 1044, fig. 289.

TYPE LOCAlity: "Red River, Arkansas" (Oklahoma).

RANGE: Oklahoma and Texas west to southeastern California,

northeastern Mexico.

\section{Thamnophis megalops (KENNICOTT)}

Eutaenia megalops KenNicotT

Proc. Ac. Nat. Sci. Phila., 1860, p. 330.

Thamnophis megalops RUTHveN

Bull. U. S. Nat. Mus., No. 61, 1908, p. 44.

Eutaenia megalops COPE

Ann. Rep. U.S. Nat. Mus. 1898 (1900), p. 1025, fig. 278.

TYPE locality: Tucson and Sta. Magdalena, Arizona.

RANGE: The whole Mexican plateau region north to Arizona and southwestern New Mexico,and the Cocopal montanin,

Lover Cali forrivia.

Thamnophis ordinoides ordinoides (BAIRD and GIRARD)

Tropidonotus ordinoides BAIIzD and GIRARD

Proc. Ac. Nat. Sei. Phila., Yol. 6, 1852, p. 176. 
Thamnophis ordinoides RUTHVEN

Bull. U. S. Nat. Mus., No. 61, 1908, p. 147.

Eutaenia elegans ordinoides COPE

Ann. Rep. U. S. Nat. Mus. 1898 (1900), p. 1046.

TYPE LOCAlity: Puget Sound.

RANGE : Coastal region of California, Oregon and Washington.

Thamnophis ordinoides elegans (BAIrd and Girard)

Eutainia elegans BAIRD and GIRARD

Cat. N. Amer. Rept., Pt. 1, 1853, p. 34.

Thamnophis ordinoides elegans RUTHVEN

Bull. U. S. Nat. Mus., No. 61, 1908, p. 138.

Eutaenia elegans elegans COPE

Ann. Rep. U. S. Nat. Mus. 1898 (1900), p. 1036, fig. 284.

ty ye locality : El Dorado County, California.

RANGE: New Mexico northward to Wyoming westward to the

Pacifie coast states. Sierra hemate elopta of hemper ars

Thamnophis proximus ( $\mathrm{SAY}$ )

Coluber proximus $\mathrm{S}_{\mathrm{AY}}$

Long's Exp. Rocky Mts., Vol. 1, 18:3, p. 187.

Thamnophis proxima GaRmaN

Bull. Essex Inst., Vol. 24, 1892, p. 105.

Thamnophis sauritus proximus RUTHVEN

Bull. U.S. Nat. Mus., No. 61, 1908, p. 98.

Eutaenia proxima CoPE

Ann. Rep. U. S. Nat. Mus. 1898 (1900), p. 1029, fig. 277.

TYPE LOCALITY : Stone quarry on west side of Missouri River,

3 miles above the mouth of Boyer's River.

RANGE: Nebraska, northern Iowa and southern Wisconsin south

to Louisiana and Texas and through Mexico to the coastal region of Yucatan and British IIonduras.

Thamnophis radix (BAIRD and GIRARD)

Eutainia radix BAIRD and GIRARD

Cat. N. Amer. Rept., Pt. 1, 1853, p. 34.

Thamnophis radix JoRDAN

Man. Vertebr. Anim. North. U. S., 8 Ed., 1899, p. 193.

Eutaenia radix COPE

Ann. Rep. U. S. Nat. Mus. 1898 (1900), p. 1026, fig. 279.

TYPE LOCALITY: Racine, Wisconsin.

RANGE: The Great Plains from Kansas and Missouri to the

Canadian Northwest.

\section{Thamnophis sackenii (KENNICOTT)}

Eutaenia sackenii KeNNICOTT

Proc. Ac. Nat. Sci. Phila., 1859, p. 98. 
Thamu ophis ondino; der ragrans(Baind an Inaw). Eutaenia ragramo Bámo aur Isinais.

Cat. Ar. Amen. Thept. Pout 1, 1853,p.35

Thanenuphis oridinoides rap rans rau Denbugh an S/ su n Proe. Calif., Acas. Sci, Ser. $\frac{5}{1}$ Vol. 8, $1918, p .240$.

Type Pocacila: Caligorria.

Range: Easteru traikuy lon and orejox, Sobko, sonit it nothern Arigone and Eastern Californio; Sau Peiro Martir Burnutarios, firme Califormia

2 Thcumurphis orvinoides afratus (Kenniest) Entainia atrala Kermic oft U.S. Pacific R.R. Surv, val. $1=, p t .2,1860,9.296$

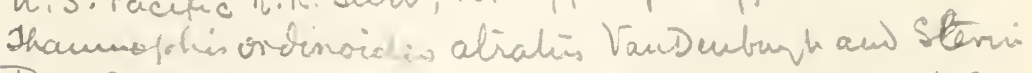
Proc. Calie acad. Sci. Ser. 4, vop 8, 1918, p.224, pl. 0 . Ty/u lvealiti. Califoriun Raupe: Craseryit of California form DeChorte

Thannophis ortinoides biscut at us (Cope).

Eutainia biscutaxa Core

Proc, acas. Tat Ser. Phile, 1883, p.21,

Thannowghis ortinoide biscutax no Van Denburg Land Slarm Pave. Cahp. Ae.d. Sci, Ser. 4, vol. 8, 1918,p.245,pl.11. Type: Pscaliti-: Keamait Cuke, uregon.

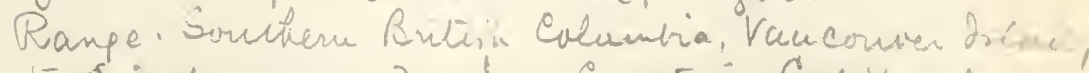
15 Siskiyon and Morke. Connter, Califernia.

Thamuophi ordenvi tes conchï (Kennicott). Eutainia conchio Kernicatt. U.S. Pacufue RR. Sum, vol 10, Pt. 4, 1859,/0.10.

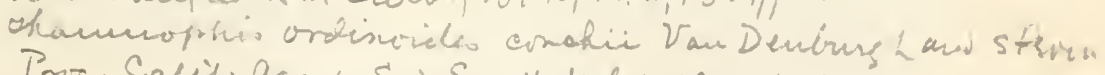
Prve. Calif. acad Sci. Ser 4, Vol. 111 1921,1.24 Type lvaculiti pitt Rire, Califarma.

Raxpe: Suer amentos uou San foaquan vallep on Call Kornia Sastwant ose the Aleirra haraite into Domfix and ivashae conceties, herada 
Thamuophins sertalis infermales (Blainelle).

Coluber infernatio Brainville.

hous ann. Mus. Paris, vol 4, 1835,p. 291, pl. 26, tiq. 3-3a.

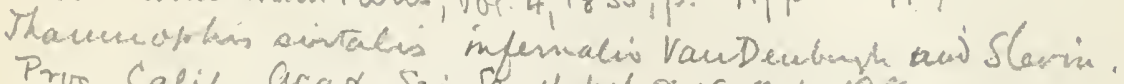

Pros. Calif. Acad. Sci. Sen. 4, 101.8,1918, p.198.

Tyke Pocality: Caleformia.

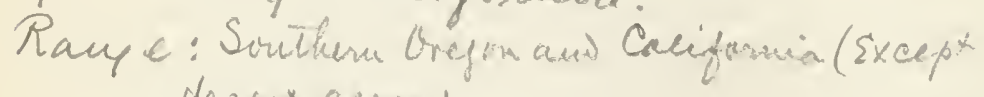
deserk areas). 
Thamnophis sauritus sackeni RutuveN

Bull. U. S. Nat. Mus., No. 61, 1908, p. 10\%.

Eutaenia sackenii COPE

Ann. Rep. I. S. Nat. Mus. 1898 (1900), p. 1019, fig. 275.

TYPE LOCALITY: Florida.

RANGE: The entire state of Florida, and southern Mississippi.

Thamnophis sauritus (LinNÉ)

RIBBON SNAKE

Coluber saurita LiNxí

Srst. Nat., Ed. 12. Vol. 1, 1766, p. 385.

Thamnophis saurita STEJNEGER

N. Amer. Fauna, No. 7, 1893, p. 210 .

Eutaenia saurita Cope

Ann. Rep. U. S. Nat. Mus. 1898 (1900), p. 1090, fig. 276.

TYPE LOCALITY: Carolina.

RANGe: Michigan, Ontario and Maine, south to Mississippi, Alabama and Georgia.

- Thamnophis sirtalis sirtalis (LINNÉ)

Coluber sirtalis LinNé

Syst. Nat., Ed. 10, Vol. 1, 1758, p. 229.

Thamnophis sirtalis GARMAN

Bull. Essex Inst., Vol. 24, 1892, p. 10!.

Eutaenia sirtalis COPE

Ann. Rep. I. S. Nat. Mus. 1898 (1900). p. 1064, figs. 298-303.

TYPE LOCALITY: Canada.

RANGE: Eastern United States and Canada north to about the 50 th degree of latitude west to Minnesota, Wisconsin and Missouri.

\section{Thamnophis sirtalis concinnus (HALLOWLLL)}

Tropidonotus concinnus Halloweld

Proc. Ac. Nat. Sci. Phila., Vol. 6, 1859, p. 189.

Thamnophis sirtalis concinnus RUTHVEN

Bull. U.S. Nat. Mus., No. 61, 1908, p. 173.

Eutaenia sirtalis concinna COPE

Ann. Rep. U. S. Nat. Mus. 1898 (1900), p. 1081, fig. 306.

TYPE LOCALITY: Oregon.

RANGE: Coast region of southwestern British Columbia, Vancouver Island, Washington and Oregon, west of the Sierra Nevada.

\section{Thamnophis sirtalis parietalis ( $\mathrm{SAY}$ )}

Coluber parietalis $\mathrm{S}_{\mathrm{AY}}$

Long's Exp. Rocky Mts., Vol. 1, 1899, p. 186. 
Thamnophis sirtalis var. parietalis JoRDAN

Man. Vertebr. Anim. North. U. S., 8 Ed., 1899, p. 193

Eutaenia sirtalis parietalis CopE

Ann. Rep. U. S. Nat. Mus. 1898 (1900), p. 107\%, fig. 304.

TYPE LOCALITY: Stone quarry on west side of Missouri River,

3 miles above the month of Boyer's River.

RANGE: Missouri to the coast of California, northward in Oregen

and Washington-only east of the Sierra-Nevada, Manitoba.

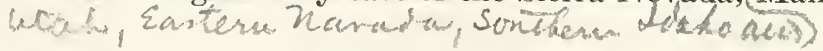

\section{Coniophanes (GENUS)}

Cope, Proc. Ac. Nat. Sci. Phila., 1860, p. 248

TYPE: fissidens

\section{Coniophanes imperialis (BAIRD)}

Taeniophis imperialis BAIRD

U. S. Mex. Bound. Surv., Vol. 2, 1859, Rept., p. 23, pl. 19, fig. 1. Coniophanes imperialis COPE

Proc. Ac. Nat. Sci. Phila., 1861, p. 74.

Coniophanes imperialis COPE

Ann. Rep. U. S. Nat. Mus. 1898 (1900), p. 1097, fig. 313.

TYPE LOCALITY: Brownsville, Texas.

RANGE: Sontheastern Texas to Vera Cruz.

\section{Leptodeira ( $G E N U S)$}

Fitzinger, Syst. Rept., 1843, p. 27

TYPE: annulata

Leptodeira septentrionalis (КеNNICOTT)

Dipsas septentrionalis KeNNicotT

U. S. Mex. Bound. Surv., Vol. 2, 1859, Rept., p. 16, pl. 8, fig. 1.

Leptodeira septentrionalis STEJNEGER

Proc. U. S. Nat. Mus., Vol. 14, 1891, p. 505.

Sibon septentrionale CoPE

Ann. Rep. U. S. Nat. Mus. 1898 (1900), p. 110\%, fig. 316.

TyPE Locality: Matamoros, Mexico and Brownsville, Texas.

RANGE: Southern Texas and northeastern Mexico.

\section{Trimorphodon ( $G E N U S)$}

Cope, Proc. Ac. Nat. Sci. Phila., 1861, p. 297

TYPE: lyrophanes

Trimorphodon lyrophanes (COPE)

Lycodon lyrophanes Cope

Proc. Ac. Nat. Sci. Phila., 1860, p. 343. 
- 
Tantillas nigricepos Kermicott Jantillar niguicepr Kannie.tt Danc, aend. hat sa. Phila, 1860,10.328 Type trealib-: Fort Bliss, hew Indepico ans turiamer t. 'hucecen, Texas.

Range Eastern and Central Arizona, hew Mupico aud Texm. 
Trimorphodon lyrophanes $\mathrm{COPE}$

Proc. Ac. Nat. Sci. Phila., 1861, p. 297.

Trimorphodon lyrophanes COPE

Amn. Rep. U. S. Nat. Mus. 1898 (1900), p. 1102, fig. 314.

TYPE LOCALity: Cape St. Lucas, Lower California.

RANGE : Southern Arizona and Lower California.

\section{Tantilla $(G E N U S)$}

Baird and Girard, Cat. N. Amer. Rept., Pt. 1, 1853, p. 131

TYPE: coronata

Tantilla coronata BAIRD and GIRARD

Tantilla coronata BAIRD and GIRARD

Cat. N. Amer. Rept., Pt. 1, 1853, p. 131.

Tantilla coronata COPE

Ann. Rep. U. S. Nat. Mus. 1898 (1900), p. 1114, fig. 320.

TYPE LOCAlity: Kemper County, Mississippi.

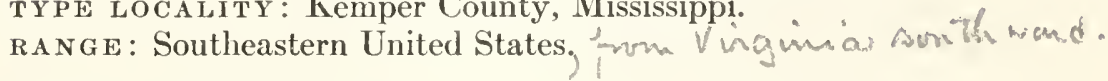

Tantilla eiseni SteJNEGER

Tantilla eiseni STEJNEger

Proc. U. S. Nat. Mus., Vol. 18, April 16, 1896, p. 117.

TyPE LOCAlity: Fresno, California.

RANGE: Southern California.

Tantilla gracilis BAIRD and GIRARD

Tantilla gracilis BAIRD and GIRARD

Cat. N. Amer. Rept., P't. 1, 1853, p. 132.

Tantilla gracilis $\mathrm{COPE}$

Ann. Rep. U. S. Nat. Mus. 1898 (1900), p. 1111, fig. 318.

TYPE LOCALITY : Indianola, Texas.

RANGE: Missouri to Texas.

\section{Tantilla planiceps (Blasinville)}

Coluber planiceps Blainville

Nouv. Ann. Mus. Hist. Nat. Paris, Vol. 4, 1835, p. 294, pl. 27, figs.

3-3B.

Tantilla planiceps Cope

Ann. Rep. U. S. Nat. Mus. 1898 (1900), p. 1113.

Tantilla nigriceps $\mathrm{Cops}$

Ann. Rep. U. S. Nat. Mus. 1898 (1900), p. 1113, fig. 319.

TYPE LOCALITY: California.

RANGE: Kansas to Texas and westward to California, and Lower

California.

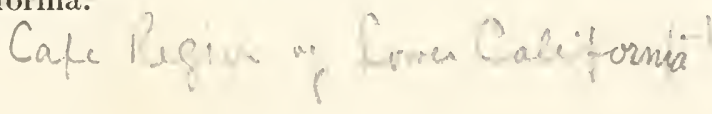


Tantilla wilcoxi STEJNEgeR

Tantilla wilcoxi STEJNEGER

Proc. U. S. Nat. Mus., Vol. 25, Sept. 8, 1902, p. 156.

TyPe LOCAlity : Fort Huachuca, Arizona.

RANGE: Southern Arizona.

\author{
ELAPIDAE (FAMILY) \\ Micrurus ( $G E N U S)$ \\ WAGLER in Spix, Serp. Brasil., 1824, p. 48 \\ TYPE: spixii \\ CORAL-SNAKES
}

Micrurus euryxanthus (KennicotT)

Elaps euryxanthus KenNicotT

Proc. Ac. Nat. Sci. Phila., 1860, p. 337.

Elaps euryxanthus CopE

Ann. Rep. U. S. Nat. Mus. 1898 (1900), p. 1125, fig. 324.

TYPE LOCALITY: Sonora.

RANGE: Soythern New Mexico, Arizona and northern Mexico;

Micrurus fulvius (LINNÉ)

Coluber fulvius Linné

Syst. Nat., Ed. 12, Vol. 1, 1766, p. 381.

Elaps fulvius Cope

Ann. Rep. U. S. Nat. Mus. 1898 (1900), p. 1120, figs. 321, 322.

TYPE LOCALITY : Carolina.

RANGE: South Carolina and Mississippi to Florida, the Gulf

States, Mexico and Central America.

\title{
CROTALIDAE (FAMILY) \\ Agkistrodon ( $G E N U S)$
}

Beauvors, Trans. Amer. Philos. Soc., Vol. 4, 1799, p. 381

TYPE: mokasen

Agkistrodon mokasen Beauvois

COPPERHEAD

Agkishodon ${ }^{\mathrm{I}}$ mokasen BeAUvors

Trans. Amer. Philos. Soc., Vol. 4, 1799, p. 370.

Ancistrodon contortrix COPE

Ann. Rep. U. S. Nat. Mus. 1898 (1900), p. 1135, fig. 326. 
$a+b$

Hydus peations ef rand 1922? 

TYPE LOCALITY: America.

RANGE: Massachusetts southward to northern Florida, westward to Illinois, Arkansas and Texas.

Agkistrodon piscivorus (LACÉ Pène)

COTTON-MOLTH MOCCASIN

Crotalus piscivorus LACÉPÈDE

Hist. Nat. Serp., Vol. ‥ 1789, table méthod., p. 130.

Ancistrodon piscivorus COPE

Ann. Rep. U. S. Nat. Mus. 1898 (1900), p. 1133, fig. 325.

TYPE LOCALITY : Carolina.

RANGE: Virginia to Florida and the Gulf States.

\section{Sistrurus (GENUS)}

Garman, Mem. Mus. Comp. Zoöl., Vol. 8, Pt. 3, 1883, p. 110 TYPE: miliarius

Sistrurus catenatus catenatus (RAFINESQUE)

MASSASAUGA

Crotalinus catenatus RAFINESQUE

Amer. Month. Mag. Crit. Rev., Vol. 4, No. 1, Nov. 1818, p. 41.

Sistrurus catenatus Garmax

Mem. Mus. Comp. Zoöl., Vol. 8, Pt. 3, 1883, p. 176.

Sistrurus catenatus catenatus Cope

Ann. Rep. U. S. Nat. Mus. 1898 (1900), p. 1146, fig. 399.

TYPE LOCALITY: Prairies of the upper Missouri.

RANGE: Western New York, through Ohio to Nebraska, north-

ward into Michigan and Ontario, southward to Kansas.

Sistrurus catenatus edwardsii (BAIRD and GIRARD)

Crotalophorus edwardsii BAIRD and GIRARD

Cat. N. Amer. Rept., Pt. 1, 1853, p. 15.

Sistrurus catenatus edwardsii STEJNEGER

Ann. Rep. U. S. Nat. Mus. 1893 (1895), p. $\$ 16$.

Sistrurus catenatus edwardsii CopE

Ann. Rep. U. S. Nat. Mus. 1898 (1900), p. 1144, fig. 328.

TYPE LOCALITY: Tamaulipas, Mexico.

RANGE: Oklahoma southward through western Texas to northeastern Mexico.

Sistrurus miliarius (LINNÉ)

GROUND RATTLER

Crotalus miliarius LinNÉ

Syst. Nat., Ed. 12, Vol. 1, 1766, p. 372. 
Sistrurus miliarius GARMAN

Mem. Mus. Comp. Zoöl., Vol. 8, Pt. 3, 1883, p. $17 \%$.

Sistrurus miliarius COPE

Ann. Rep. U. S. Nat. Mus. 1898 (1900), p. 1141, fig. 327. TYPE LOCALITY: Carolina.

RANGE: North Carolina to Florida westward to Oklahoma and

Texas, northward in the Mississippi basin to Arkansas.

\section{Crotalus ( $G E N U S)$}

Lrnne, Syst. Nat., Ed. 10, Vol. 1, 1758, p. 214

TYPE: horridus

RATTLESNAKES

\section{Crotalus adamanteus Beauvors}

DIAMOND-BACK RATTLER

Çrotalus adamanteus BeAuvoIs

Trans. Aner. Philos. Soc., Vol. 4, 1799, p. 368.

Crotalus adamanteus adamanteus Cope

Ann. Rep. U. S. Nat. Mus. 1898 (1900), p. 1161, fig. 333.

TYPE LOCALITY: United States.

RANGE : Southern North Carolina to Florida, westward to Louisiana and Arkansas.

\section{atrox}

\section{Crotalus atrox BAIRD and Girard}

Crotalus atrox BAIRD and GIRARD

Cat. N. Amer. Rept., Pt. 1, 1853, p. 5.

Crotalus adamanteus atrox COPE

Ann. Rep. U. S. Nat. Mus. 1898 (1900), p. 1164, fig. 334.

Crotalus adamanteus scutulatus CopE

Ann. Rep. U. S. Nat. Mus. 1898 (1900), p. 1158, fig. 332.

TYPE LOCAlity: San Pedro, Texas.

RANGE: Texas and northern Mexico to Arizona, also Lower

California.

Crotalus cerastes HALLOWELL SIDEWINDER

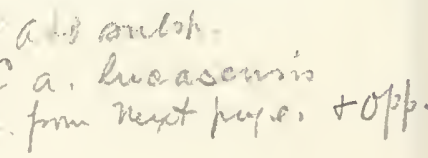

Crotalus cerastes Hallowell

Proc. Ac. Nat. Sci. Phila., Vol. 7, 1854, p. 95.

Crotalus cerastes Cope

Ann. Rep. U. S. Nat. Mus. 1898 (1900), p. 1196, fig. 347.

TYPE LOCALITy: Mojave Desert.

RANGE: Southern California, southern Nevada, Arizona, southwestern Utah.

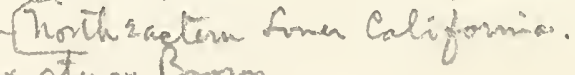

cretahe atrox atuox Bromen

Roc. Acad. hat. Sci. Phila.,1901,p.103 
Crotalus atrox elegans schuidt crotalus atrox eleqaus schuidt Buse aures. Mhs. hax. 1tist. vol 46, Dec. $71922, p .699$. Type lucality: Angel de la Guardia (sic) bland, forer califormia.

Range: save. 
atrox

Crotalus hecasensis Vou Denburgh Crotalus Rueasens is Van Denbungh.

Proe. Calif. Acad. Sei. Ser. 4, vol. 10, Gug. L, 1920, 1p. 29

Jyppelucality: Ggua Caliente, Lome Califimia.

Range: Southare comer Califorrice.

Crotalus atiox hecasensis Schmidt

Bull, Amen. Inwi, hat. Hist, it 6, sec.p, $19,2, p .698$

rol. 
Crotalus confluentus SAY

PRAIRIE RATTLER

Crotalus confluentus $\mathrm{S}_{\mathrm{A}} \mathrm{Y}$

Long’s Exp. Rocky Mts., Vol. 2, 1823, p. 48.

Crotalus confluentus COPE

Ann. Rep. U. S. Nat. Mus. 1898 (1900), p. 1169, figs. 336-338.

TYPE LOCALITY: "Valley of the Arkansa "near Bell's Springs, Colorado.

RANGE: The Great Plains, from about the 96th Meridian to the Rocky Mountains and from Southern Canada to Texas.

Crotalus enyo (Cope)

Caudisona enyo Cope

Proc. Ac. Nat. Sci. Phila., 1861, p. 293.

Crotalus enyo CoPE

Bull. U. S. Nat. Mus., No. 1, 1875, p. 33.

Crotalus enyo Cope

Ann. Rep. U. S. Nat. Mus. 1898 (1900), p. 1189, fig. 343.

TYPE LOCALITY: Cape St. Lucas, Lower California.

RANGE : Extreme southern Lower California.

Crotalus exsul Garman

Crotalus exsul Gariran

Nem. Mus. Comp. Zoöl., Vol. 8, Pt. 3, 1883, p. 11 .

Crotalus ruber Cope

Ann. Rep. U. S. Nat. Mus. 1898 (1900), p. 1167, fig. 335.

TYPE LOCALITY: Cedros Island, Lower California,

RANGE: Southern and Lower Californiaren Cespo Szlas.

Crotalus horridus Linxé

znotickastion

BANDED RATTLESNAKE, BLACK RATTLESNAKE, TIMBER RATTLESNAKE, CANEBREAK RATTLESNAKE (FOR VARIOUS VARLANTS IN COLORATION)

Crotalus horridus LinnÉ

Syst. Nat., Ed. 10, Vol. 1, 1758, p. 214.

Crotalus horridus COPE

Ann. Rep. U. S. Nat. Mus. 1898 (1900), p. 1185, fig. 342.

TYPE LOCALITY : America.

RANGE: Maine to Georgia, westward to Great Plains.

Crotalus lepidus (KennicotT)

Caudisona lepida KennicotT

Proc. Ac. Nat. Sci. Phila., 1861, p. 206.

Crotalus lepidus Cope

Proc. Ac. Nat. Sci. Phila., 1883, p. 13. 
Crotalus lepidus Cope

Ann. Rep. U. S. Nat. Mus. 1898 (1900), p. 1191, fig. 344.

type locality: Presidio and Eagle Pass, Texas.

RANGE: Border region in Texas, New Mexico, Arizona and adjacent Mexico.

\section{Crotalus mitchellii (COPE)}

Caudisona mitchellii Cope

Proc. Ac. Nat. Sci. Phila., 1861, p. 293.

Crotalus mitchellii Cope

Wheeler's Surv. West 100 Mer., Vol. 5, 1875, p. 535.

Crotalus mitchellii Cope

Ann. Rep. U. S. Nat. Mus. 1898 (1900), p. 1193, figs. 345, 346.

TYPE lOCAlity: Cape St. Lucas, Lower California.

rANGE: Arizona, Colorado Desert to southern Lower California.

Crotalus molossus BAird and Girard

Crotalus molossus BAIRD and Girard

Cat. N. Amer. Rept., Pt. 1, 1853, p. 10.

Crotalus molossus Cope

Ann. Rep. U. S. Nat. Mus. 1898 (1900), p. 1154, fig. 330.

TyPe locality : Fort Webster, New Mexico.

RANGE: Southern Texas to southern Arizona, also northern Mexicorand Aaw Eateban Slawd.

\section{Crotalus oregonus HoLBrook}

Crotalus oreganus НоцвRоок

N. Amer. Herp., Ed. 1, Vol. 4, 1840, p. 115, pl. 29 [=24]

Crotalus confluentus lucifer Cope

Ann. Rep. U. S. Nat. Mus. 1898 (1900), p. 1176, fig. 339.

TYPE LOCALITY: Columbia River.

RANGE: British Columbia to southern California, western Idaho,

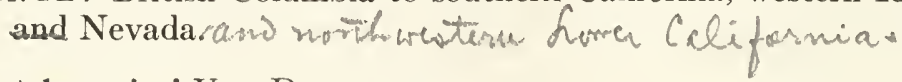

Crotalus pricei VAN Denburgh

Crotalus pricei $\mathrm{V}_{\text {AN }}$ Denburgh

Proc. California Ac. Sci., Ser. 2, Vol. 5, 1895, p. 856.

Crotalus pricei COPE

Ann. Rep. U. S. Nat. Mus. 1898 (1900), p. 1184.

TyPE Locality : Huachuca Mts., Arizona.

RANGE: Southern Arizona and the adjacent region in Mexico.

Crotalus tigris KENNICOTT

Crotalus tigris KennicotT

U. S. Mex. Bound. Surv., Vol. 2, 1859, Rept., p. 14, pl. 4. 
Cratalus golchami Schniclt Crotalus goebuani Sehmidt Bull. Amen. Ulus. hat tist vol. 46, Dec.7,1922, p. 701. Type Pveality: Ee Piñon, Comer California. Raupe' same.

$\checkmark$ Cratalus tor tugensis rau Deubush a Slerm Crotalus tortugensis ranteubing + Slevin. Prove. Culip, Acad. Sei., Ser.4, Vol.11, Dec.171921,j.398. The lorality: Intuga blaw, Lrra California. Rampe: same. 


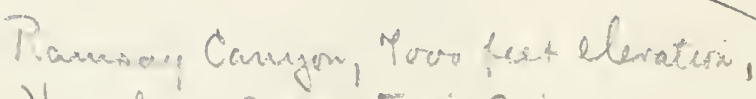

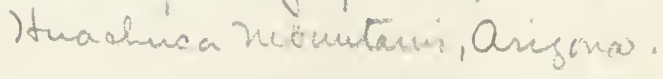


Crotalus tigris COPE

Ann. Rep. U. S. Nat. Mus. 1898 (1900), p. 1181 , fig. 341.

TYPE LOCALITY: Deserts of the Gila and Colorado Rivers, Arizona.

RANGE: Southern California, southern Nevada, and southern Arizona.

$\checkmark$ Crotalus willardi MЕek

Crotalus willardi MEEK

Field Columb. Mus. Publ. Zoöl., Vol. 7, No. 1, Jan. 1906, p. 18, pl. 3. TYPE LOCALITY : 'Tombstone, Arizena.

RANGE: Santa Rita Mountain Region, Arizona, and northern Mexico.

\section{SYNAPSIDA (SUBCLASS)}

Osborn, Science (N. S.), Vol. 17, Feb. 13, 1903, p. 276

\section{TESTUDINATA (ORDER)}

Oppel, Ordn. Rept., 1811, p. 3

KINOSTERNIDAE (FAMILY)

Kinosternon ( $G E N C S)$

SpIx, Testud. Brasil., 1824, p. 17

TYPE: longicaudatum $=$ scorpioides.

STINK-POTS, MUSK-TURTLES OR MUD-TURTLES

Kinosternon baurii GarMaN

Cinostermum baurii $\mathrm{G}_{\mathrm{ARMAN}}$

Bull. Essex Inst., Vol. 29, 1891, p. 1+1.

TyPE LOCALITy: Key West, Florida.

RANGE: Florida.

\section{Kinosternon carinatum (GRA')}

Aromochelys carinata GraY

Proc. Zool. Soc. London, 1855 (Jan. 22, 1556), p. 199.

TYPE LOCALITY : Louisiana.

RANGE: Western Georgia to Louisiana.

Kinosternon flavescens (AG.Assiz)

Platythyra flavescens AGassiz

Contr. Nat. Hist. U. S., Vol. 1, 1857, p. 430; Vol. 2, pl. 5, figs. 12-15. 
Cinosternum flavescens CoPE

Bull. U. S. Nat. Mus., No. 1, 1875, p. 52.

TyPE LoCAlity: Texas, Arizona.

RANGE: Texas, north to Kansas and Colorado, west to Arizona.

Kinosternon odoratum (LATREILLE)

Testudo odorata LATreille

Hist. Nat. Rept., Vol. 1, 1802, p. 122.

Kinosternon odoratum GraY

Syn. Rept., 1831, p. 35.

TYPE LOCALITY: Carolina.

RANGE: Eastern and southern United States from Canada south;

west to western Missouri and southern Texas.

Kinosternon sonoriense Le Conte

Kinostermum sonoriense LE CONTE

Proc. Ac. Nat. Sci. Phila., 1854, p. 184.

ty PE LOCAlity: Tucson, Arizona.

RANGE: Southwestern Texas through southern New Mexico and Arizona into southeastern California; northern Mexico.

\section{Kinosternon steindachneri SiEBENRock}

Cinosternum steindachneri SIEBENRock

Zool. Anz., Vol. 30, Oct. 16, 1906, p. 727.

type locality: Orlando, Florida.

RANGE : Central Florida.

Kinosternon subrubrum subrubrum ${ }^{1}$ (LACÉPÈDE)

Testudo subrubra LACÉPÈDE

Hist. Nat. Quadr. Ovip. Serp., Vol. 1, 1788, synops. méth., tabl. betw. pp. 618 and 619 (comp. p. 132).

TYPE LOCALITy: Pennsylvania.

RANGE: Eastern United States, exclusive of peninsular Florida, west to Indiana and southeastern Illinois and 'Tennessee.

\section{Kinosternon subrubrum hippocrepis (GRAY)}

Kinosternon hippocrepis GraY

Proc. Zool. Soc. London, 1855 (Jan. 22, 1856), p. 198.

TYPE LOCALITY: New Orleans, Louisiana.

RANGE: Southern Alabama to Texas, north in the Mississippi

Valley to southeastern Missouri.

This is the species usually known as Kinosternon pensiltanicum (GMELIN) 1789. Both names are based on the same figure in Edward's "Glanures". 

Chelydra usceola Strineger in Florida suapper

chely cha osceola Stijuequ, Proc. Biol. Soc. Nash. Vol.31, 1918, p. 89 . (fune 24)

Tyluelveality: Clearwater, Pinellas, Co., Fla.

Range: Pinellas, Osceon, Brevard 'Polk Cos. Fla.

曹. mi 1919 S.to kew River, Bromars Co. 


\section{CHELYDRIDAE (FAMILY)}

Macrochelys (GENUS)

Gray, Proc. Zool. Soc. London, 185.5 (Jan. 29, 185(i), p. 200

TYPE: temminckii

\section{Macrochelys temminckii (Ноцвиоок)}

ALLIGATOR-SNAPPER

Chelonura temminekii HoLBrook

N. Amer, Herp., Ed. 1. 18t0, Vol. 4, p. 29, pl. 4.

Macrochelys temminelii GraY

Proe. Zool. Soe., Loudon, 185.5 (Jan. Q9. 1856) p. 200.

TYPE LOCALITY: Mississippi River and tributaries.

RANGE: Texas east to southeastern Georgia and northern Florida, north in the Mississippi basin to northern Missouri.

\section{Chelydra (GENTS)}

Schweigger, Königsberg. Areh. Naturw. Math., Vol. 1, 1819, Pt. 3. p. 292 TY PE: serpentina

Chelydra serpentina (LinNÉ)

COMMON SNAPPER

Testudo serpentina LINNÉ

Syst. Nat.. Ed. 10. Vol. 1, 1758, p. 199.

Chelydra serpentina Schweigger

Königsberg. Arch. Naturw. Math., Yol. 1, 1812, Pt. 3, p. 293.

TYPE LOCALITY: "Warmer regions."

RANGE: Eastern North America from southern Canada to the

Gulf of Mexico and west to the Rocky Mountains.

\section{TESTUDINIDAE $(F A M I L Y)$}

Clemmys (GENUS)

Ritgen, Nova Acta Ac. Leopoll.-Carol., Vol. 14, Pt. 1. 1828, p. 272 TYPE: punctata $=$ guttata

Clemmys guttata (Schneider)

SPOTTED TURTLE

Testudo guttata ScIINEIDER

Schr. Ges. Naturf. Freunde Berlin, Vol. 10, 1792, p. 264.

Clemmys guttata STrauci

Mém. Ac. Sci. St. Pétersbourg, Ser. 7, Vol. 5, No. 7, 1862, p. $10 \tau$. 
TYPE LOCALITY: Unknown.

RANGE: Eastern United States from Maine to northern Florida; in northern part west and north to southern Michigan.

Clemmys insculpta (Le Contr)

WOOD-TURTLE

Testudo insculpta Le Conte

Ann. Lyyc. Nat. Hist. New York, Vol. 3, 1830, p. 112.

Clemmys insculpta Straucir

Mém. Ac. Sci. St. Pétersbourg, Ser. 7, Vol. 5, No. 7, 1862, p. 104.

TYPE LOCALITY: Northern (United) States.

RANGE: Eastern United States from Maine to the District of

Columbia, west to Michigan and Wisconsin; southwestern

Ontario.

\section{Clemmys marmorata (BAIRD and GirARD)}

Emys marmorata BAIRD and Girard

Proc. Ac. Nat. Sci. Phila., Vol. 6, 1852, p. $17 \%$.

Clemmys marmorata Strauch

Mém. Ac. Sci. St. Pétersbourg, Ser. 7, Vol. 5, No. 7, 1862, p. 108.

TYPE LOCALitY: Puget Sound, Washington.

RANGE: Pacific Slope from Puget Sound to San Diego, California.

\section{Clemmys muhlenbergii (SchокрғF)}

Testudo muhlcnbergii SсноерғF

Hist. Testud. Pt. 6, 1801, p. 132. pl. 31.

Clemmys muhlenbergii Strauch

Mém. Ac. Sci. St. Pétersbourg, Ser. 7, Vol. S, No. 13, 1865, p. 67.

TyPE LOCALITY: Lancaster, Pennsylvania.

RANGE: New Jersey, soutlieastern corner of Pennsylvania and of

New York, north-to Rhode Island,

Cantiva.

\section{Clemmys nuchalis DUNN}

Clemmys nuchalis DunN

Bull. Amer. Mus. Nat. Hist., Vol. 37, 1917, p. 624, pl. 61.

TYPE LOCALITY: Yonahlossee Road, about 3 miles from Lin-

ville, North Carolina.

RANGE: Brevard and Linville, North Carolina. 




\author{
Emys ( $G E N U S)$ \\ Dumérit, Zool. Anal., 1806, p. 76 \\ TY $\mathrm{P}$ : Europea
}

\title{
Emys blandingii (Holbrook)
}

BLANDING'S TURTLE

Cistudo blandingii HoLBrook

N. Amer. Herp., Ed. 1, Vol. 3, 1838, p. 35, pl. 5.

Emys blandingii Strauch

Mém. Ac. Sci. St. Pétersbourg, Ser. 7, Vol. 5, No. 7, 1862, p. 28.

TyPE LOCALity: Fox River, Illinois.

RA NGE: Northern Illinois and Indiana, west and north to Minne-

sota, Miehigan and east to New England; Long Island.

\section{Terrapene (GENUS)}

Merreu, Tent. Syst. Amphib., 1890, p. 27

TYPE: clausa $=$ carolina

BOX-TURTLES

Terrapene carolina carolina (LINNÉ)

Testudo carolina LINNÉ

Syst. Nat., Ed. 10, Vol. 1, p. 198.

Terrapene carolina BELI.

Zool. Journ., Vol. 2, 1825, p. 309.

TYPE LOCALITY: Carolina.

RANGE: Eastern United States from Maine to Georgia, west to

Tennessee, western Illinois and northwards to central Michigan.

\section{Terrapene carolina triunguis (AGASSIZ)}

Cistudo triunguis AGASsiz

Contr. Nat. Hist. U. S., Vol. 1, 1857, p. 445.

Terrapene carolina triunguis STRECKER

Proc. Biol. Soc. Washington, Vol. 23, 1910, p. 121.

TYPE LOCALITY: New Orleans, Louisiana.

RANGE: Coastal plain of the Gulf of Mexico, north in the Mississippi Basin to Missouri, west into Oklahoma and southern Texas.

\section{Terrapene major (AgAssiz)}

Cistudo major AGAssiz

Contr. Nat. Hist., U. S., Vo!. 1, 1857, p. 445.

Terrapene major 13 AUR

Science, N. S., Vol. 17, April 3, 1891, p. 190. 
TyPE LOCALITy: Mobile, Alabama; Florida.

RANGE: Florida, north into southern Georgia, west into southeastern Texas.

\title{
Terrapene ornata (AGASSIz)
}

Cistudo ornala Agassiz

Contr. Nat. Hist. U. S., Vol. 1, 1857, p. 445.

Terrapene ornata $\mathrm{B}_{\mathrm{AUR}}$

Science, N. S.. Vol. 17, April 3, 1891, p. 191.

TYPE LOCALITY: The Upper Missouri and Iowa.

RANGE: Indiana, Illinois, and territory between the Missouri and Mississippi Rivers and the Rocky Mountains from the Yellowstone River in the north to the Gulf of Mexico in the south, southern New Mexico and Arizona and northern Mexico.

\author{
Malaclemys ( $G E N U S)$ \\ Gray, Cat. Tort. Brit. Mus., 18 44 , p. 28 \\ TY PE: concentrica \\ DIAMOND-BACK TERRAPINS
}

Malaclemys centrata centrata (LATREILLE)

Testudo centrata LATREILLE

Hist. Nat. Rept, Vol. 1, 1802, p. 145.

Malaclemys centrata LoENNBERG

Proc. U. S. Nat. Mus., Vol. 17, Nov. 15, 1894, p. 319.

TYPE LOCALITY: Carolina.

RANGE: Coast strip from neighborhood of Cape Hatteras, south to Florida.

Malaclemys centrata concentrica (SHAw)

Testudo coneentrica SHaw

Gen. Zool., Vol. 3, 1802, p. 49, pl. 9.

Malaclemmys centrata concentrica W. P. HAY

Bull. U.S. Bur. Fish., Vol, 24, 1904, p. 16.

TYPE LOCALITY: Philadelphia markets.

RANGE: Coast strip from Buzzards Bay to North Carolina, Delaware and Chesapeake Bays.

Malaclemys pileata pileata (WIED)

Emys pileata WIED

Nova Acta Ac. Leopold.-Carol., Vol. 32, 1865, p. 17, pl. 1, figs. 2, 3.

Malaelemmys pileata W. P. $\mathrm{H}_{\mathrm{AY}}$

Bull. U.S. Bur. Fish., Vol. 24, 1904, p. 17.

TYPE LOCALITY: New Orleans, Louisiana.

RANGE: Coast from mouth of Mississippi River eastwards to Florida. 


Malaclemys pileata littoralis (W. P. H.AY)

Malaclemmys littoralis W. P. HAY

Bull. U.S. Bur. Fish., Vol. 24, 1904, p. 18, pls. 8, 9, 12, figs. 2, 3.

TYPE LOCALITY: Rockport, Texas.

RANGE: Coast of 'Texas and outlying islands.

Malaclemys pileata macrospilota (W. P. HAY)

Malaclemmys macrospilota W. P. HAY

Bull. U. S. Bur. Fish., Vol. 24, 1904. p. 16, pls. 6, 7, 11, fig. 1.

TYPE LOCALITY: Charlotte Harbor, Florida.

RANGE: Western coast of Florida.

\author{
Graptemys ( $G E N U S)$ \\ Ag.assiz, Contr. Nat. Hist. U.S., Vol. 1, 1857, p. 436 \\ TYPE: gcographica \\ MAP-TURTLES
}

\title{
Graptemys geographica (LE Sueur)
}

Testudo geographica Le SUEUR

Journ. Ac. Nat. Hist. Phila., Vol. 1, Pt. 1, 1817, p. 86, pl. 5.

Graptemys geographica AG.assiz

Contr. Nat. Hist. U. S.. Vol. 1, 1857, p. 436.

TYPE LOCALITY: Marsh on the border of Lake Erie.

RANGE: Mississippi Valley north to Iowa, northern Illinois, southern Michigan east to Pennsylvania and New York as far as Lake Champlain; eastern Tennessee and southwestern Virginia, west to southwestern Missouri, eastern Oklahoma and northeastern Texas.

\section{Graptemys pseudogeographica pseudogeographica (GRAY)}

Emys pseudogeographica GraY

Syn. Rept., 1831, p. 31 ("Le Sueur" in synomymy).

Graptemys pseudogeographica GRAY

Ann. Mag. Nat. Hist., Ser. 3, Vol. 12, Sept. 1863, p. 180.

TYPE LOCALITY: Wabash River, New Harmony, Indiana.

RANGE: Mississippi Valley, east to Alabama and Ohio, north to

Wisconsin and northern Iowa; west to eastern Kansas and

Oklahoma.

Graptemys pseudogeographica kohnii (B.AvR)

Malacoclemmys kohnii B.Aur

Science, N.S., Vol. I6, Nov. 7, 1890, p. 263.

TYPE LoCAlity: Bayou Lafourche, B. Teche, and St. Martins-

ville, Louisiana.

RANGE: Gulf strip from Pensacola to eastern Texas. 
Graptemys pseudogeographica oculifera (BAUR)

Malacoclemmys oculifera $\mathrm{B}_{\mathrm{AUR}}$

Science, N. S., Vol. 16, Nov. 17, 1890, p. 262.

TYPE LOCALity: Mandeville, Louisiana.

RANGE : Southern Louisiana.

\section{Chrysemys (GENUS)}

GraY, Cat. Tort. Brit. Mus., 1844, p. 27

TYPE: picta

PAINTED TURTLES, SOLDIER TURTLES

Chrysemys marginata marginata (Agassiz)

Chrysemys marginata AgAssiz

Contr. Nat. Hist. U. S., Vol. 1, 1857, p. 439, Vol. 2, pl. 1, fig. 6, pl. 5, figs 1-4.

TrPe LOCALIty: Wisconsin, Michigan, Indiana, Iowa.

RANGE : Central lowland, west to the Mississippi and the Missouri, north into Wisconsin, Michigan, and northern New York.

Chrysemys marginata bellii (GRAY)

Emys bellii GraY

Syn. Rept., 1831, p. 31.

TYPE LOCALITY : Unknown.

RANGE: Great plains west of the Mississippi, southwest into New

Mexico and northern Mexico; northwest into Montana, the Columbia basin, British Columbia and Vancouver Island; northeast into northeastern Minnesota and upper Michigan.

Chrysemys marginata dorsalis (AgAssiz)

Chrysemys dorsalis Agassiz

Contr. Nat. Hist. U. S., Vol. 1, 1857, p. 440.

TYPE LOCALITY: Mississippi and Louisiana.

RANGE: Mississippi Valley north to southern Missouri.

Chrysemys marginata treleasei (HuRTER)

Chrysemys treleasei HurTer

Trans. Ac. Sci. St. Louis, Vol. 20, July 28, 1911, p. 255, pl. 23, fig. 3, pl. 24

TYPE LOCALITY: St. Clair and Madison Cos., Illinois. RANGE : St. Clair and Madison Cos., Illinois.

Chrysemys picta (Schneider)

Testudo picta Schneider

Naturg. Schildkr., 1783, p. 348. 




\section{Chrysemys picta GRAY}

Cat. Shield Rept. Brî̀. Mus., I't. 1, March 8, 1856, p. 32 (part). TYPE LOCALITY: Unknown, said to have been England. RANGE: Eastern North America from New Brunswick to Georgia.

\section{Pseudemys ( GENUS)}

Grax, Proc. Zool. Soc. London, 1855 (Jan. 22, 1856), p. 197

TYPE: concinna

SLIDERS OR COOTERS

$\sqrt{ }$ Pseudemys alabamensis BAtiR

Pseudemys alabamensis BAUR

Proc. Amer. Philos. Soc., Vol. 31, July, 1893, p. 224.

TYPE LOCALITY: Mobile Bay, Alabama.

RANGE: Gulf coast from Florida to Louisiana.

Pseudemys concinna (Le Conte)

Testudo concinna Le CoNTe

Ann. Lyc. Nat. Hist. New York, Vol. 3, 1830, p. 106.

Pseudemys concinna GraY

Cat. Shield Rept. Brit. Mus., Pt. 1, March 8, 1856, p. 34.

TYPE LOCALITY: Rivers of Georgia and Carolina above Augusta and Columbia.

RANGE: Eastern rivers from Georgia to southern Maryland.

\section{Pseudemys elegans (WIED)}

Emys elegans WIED

Reise Nord-Amer., Vol. 1, P't. 4, 1838, p. 213.

Pseudemys elegans Cope

Bull. U. S. Nat. Mus., No. 1, 1875, p. 53.

ty Pe LoCALity: New Harmony, Indiana.

RANGE: Southern Texas and Mississippi basin north to Iowa, northern Indiana and Ohio.

\section{Pseudemys floridana (Le ContF)}

Testudo floridana LE Conte

Ann. Lyc. Nat. Hist. New York, Vol. 3, 1830, p. 100.

Pscudemys floridana $\mathrm{B}$ Aur

Proc. Amer. Philos. Soc., Vol. 31, 1893, p. 223.

TYPE LOCALITY: St. John's River, Florida.

RANGE: Florida into southern Georgia.

\section{$\sqrt{ }$ Pseudemys hieroglyphica (НоLBrook)}

Emys hieroglyphica НоцвRоок

N. Amer. Herp., Ed. 1, Vol. 1, 1836, p. 47, pl. 2. 
Pseudemys hieroglyphiea $\mathbf{H}$. Garman

Bull. Illinois Lab. Nat. Hist., Vol. 3, 1892, p. 231.

TYPE locality: Cumberland River, Tennessee.

RANGE: Rivers of southern Appalachians.

\section{Pseudemys ornata nebulosa (VAN Denburgii)}

Chrysemys nebulosa Van Denburgir

Proc. California Ac. Sci., Ser. 2, Vol. 5, 1895, p. 84, pls. 4-6.

TYPE LOCALITy : Los Dolores, abreast of San José Island, Lower

California.

RANGE : Southern Lower California.

Pseudemys rubriventris (LE CONTE)

Testudo rubriventris LE Conte

Amm. Lyc. Nat. Hist. New York, Vol. 3, 1830, p. 101.

Pseudemys rubriventris Loennberg

Proc. U.S. Nat. Mus., Vol. 17, Nov. 15, 1894, p. 318.

TyPE LOCALity: Delaware River, near Trenton, New Jersey.

RANGE: Atlantic coast from Florida to New Jersey; Plymouth,

Massachusetts.

$\checkmark$ Pseudemys scripta (SchoepfF)

Testudo scripta SchoepfF

Hist. Testud., Pts. 1-2, 1792, p. 16, pl. 3, figs. 4, 5.

Pseudemys scripta Jordan

Man. Vertebr. Anim. North U. S., Ed. 8, 1899, p. 209.

TYPE LOCALITY : Unknown.

RANGE: Georgia to North Carolina.

Pseudemys texana BAUR

Pseudemys texana BAUR

Proc. Amer. Philos. Soc., Vol. 31, July, 1893, p. 223.

TyPE LOCALITY : San Antonio, Texas.

RANGE: Southwestern Missouri, western Arkansas and Texas to northeastern Mexico.

\section{Pseudemys troostii (НоLвrook)}

Emys troostii HoLBrook

N. Amer. Herp., Ed. 1, Vol. 1, 1836, p. 55, pl. 4.

Pseudemys troostii COPE

Bull. U. S. Nat. Mus., No. 1, 1875, p. 53.

TYPE LOCALITY: Cumberland River, Tennessee.

RANGE: Mississippi Valley, north to northern Missouri and southern Indiana. 


Deirochelys ( $G E N U S$ )

Agassiz, Contr. Nat. Hist. U.S., Vol. 1, 185\%, p. $4+1$

TYPE: reticulata $=$ reticularia

\section{Deirochelys reticularia (LATREILLE)}

CHICKEN TLRTLE

Testudo reticularia LATREILLE

Hist. Nat. Rept., Vol. 1, 1802, p. 124.

Deirochelys reticularia GRAY

Suppl. Cat. Shield Rept. Brit. Mus., Pt. 1, 1870, p. 39 (part).

TYPE LOCALITY: Carolina.

RANGE: Coastal Plain from Beaufort, North Carolina to and including the Mississippi alluvial plain; south to central Florida.

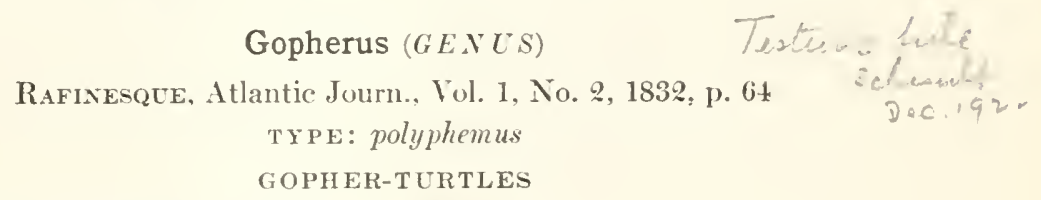

Gopherus agassizii (Cooper)

Xerobates agassizii Cooper

Proc. California Ac. Sci., Vol. 2, 1863, p. 120.

Gopherus agassizii SteJneger

N. Amer. Fauna, No. 7, May 31, 1893, p. 161.

TYPE LOCALITY: Mountains near Ft. Mojave, California.

RANGE: Deserts of southwestern Arizona, and southeastern Cali-

fornia into Nevada.

\section{Gopherus berlandieri (AGAssiz)}

Xerobates berlandieri AGAssiz

Contr. Nat. Hist. U. S., Vol. 1, 1857, p. 447, Vol. 2, pl. 3, figs. 17-19.

Gopherus berlandieri STEJNEGER

N. Amer. Fauna, No. 7, May 31, 1893, p. 161.

TYPE LOCALITY: Lower Rio Grande, 'Texas.

RANGE: Southwestern corner of Texas into northeastern Mexico.

\section{Gopherus polyphemus (DACDIN)}

Testudo polyphemus Daudin

Hist. Nat. Rept., Vol. 2, 1803, p. 256.

Gopherus polyphemus StejNeger

N. Amer. Fauna, No. 7, May 31, 1893, p. 161.

TYPE LOCALITY: Savanna and Altamaha Rivers, Georgia.

RANGE: Coast from southern South Carolina to Florida and the

Mississippi River north into southern Arkansas. 


\author{
CHELONIIDAE (FAMILY) \\ Chelonia ( $G E N U S$ ) \\ Latreille, Hist. Nat. Rept., Vol. 1, 1802, p. $2 \mathcal{Q}$ \\ TYPE: mydas \\ GREEN-TURTLES
}

Chelonia agassizii Bocourt

Chelonia agassizii BocourT

Ann. Sci. Nat., Ser. 5, Zool., Vol. 10, Pts. 1-3, 1868, p. 122.

TYPE LOCALITY: Guatemala, mouth of Nagualate in the Pacific. RANGE: Pacific Ocean, north to coasts of Lower California and southern California.

Chelonia mydas (Linné)

Testudo mydas LinNé

Syst. Nat., Ed. 10, Vol. 1, 1758, p. 197.

Chelonia mydas Schweigger

Königsberg. Arch. Naturw. Math., Vol. 1, 1812, Pt. 3, p. 412. TYPE LOCALITY: Ascension Island, etc.

RANGE: Atlantic Ocean; Gulf of Mexico, occasionally as far north

as Massachusetts.

\author{
Eretmochelys ( $G E N U S)$ \\ Fitzinger, Syst. Rept., 1843, p. 30 \\ TYPE: imbricala \\ SHELL-TURTLES, HAWKS-BILLS
}

Eretmochelys imbricata (LINNÉ)

Testudo imbricata LinNé

Syst. Nat., Ed. 12, Vol. 1, 1766, p. 350.

Eretmochelys imbricata AGASSIZ

Contr. Nat. Hist. U. S., Vol. 1, 1857, p. 381.

TYPE Locality: American Seas.

RANGE: Florida and Gulf coasts; occasionally as far north as

Massachusetts.

Eretmochelys squamosa (Girard)

Caretta squamosa Girard

Herpet. U. S. Expl. Exped., 1858, p. 442, pl. 30, figs. 1-7

Eretmochelys squamosa STEJNEGER

Bull. U. S. Nat. Mus., No. 58, 1907, p. 511.

TYPE LOCALITY: Sulu Seas and Indian Ocean.

RANGE: Pacific Ocean; coast of Lower California. 


Caretta ( $G E N U S)$

Rafinesque, Specchio Sci. (Palermo), Vol. 2, No. 9, 1 Sept., 1814, p. 66 TYPE: nasuta $=$ caretta LOGGERILAD TURTLES

\section{Caretta caretta (LINNÉ)}

Testudo caretta LinNé

Syst. Nat., Ed. 10, Vol. 1, 1758, p. 197.

Caretta caretta Stejneger

Ann. Rep. U. S. Nat. Mus. 1902 (1904), p. 715.

TYPE LOCALITY : "About the American Islands."

RANGE: Atlantic Ocean, breeding as far north as Beaufort, North

Carolina; north occasionally to coast of Massachusetts.

Caretta kempii (Garmax)

BASTARD-TURTLE

Thalassochelys (Colpochelys) kompii Garman

Bull. Mus. Comp. Zoöl., Vol. 6, 1880, p. 123.

Caretta kempii SIEBENROCK

Zool. Jahrb. Suppl., Vol. 10, Pt. 3, 1909, p. 551.

TYPE LOCALITY: Gulf of Mexico.

RANGE: Northeastern part of Gulf of Mexico north to Cape Hat-

teras, and accidentally to the coast of Massachusetts.

\section{Caretta olivacea (Eschschoutz)}

Chelonia olivacea Eschscholtz

Zool. Atlas, Pt. 1, 1829, p. 2, pl. 3.

Caretta oliracea RueppelL

Neue Wirbelth. Abyssin., Amph., 1835, p. 7.

TyPE LOCALITy: Manila Bay, P. I.

RANGE: Tropical Pacific straggling to the California coast.

\section{DERMOCHELIDAE (FAMLY)}

\section{Dermochelys ( $G E N U S)$}

Blainville, Bull. Soc. Philom. Paris, 1816, p. 111 bis (= 119) TY PE: coriacea

Dermochelys coriacea (LINNÉ)

Testudo coriacea Linné

Syst. Nat., Ed. 12, Vol. 1, 1766, p. 350.

Dermochelys coriacea Boulenger

Cat. Chel. Brit. Mus., 1889, p. 10. 
TyPE LOCALITy: Mediterranean Sea.

RANGE: Atlantic Ocean; occasionally on entire coast as far north as Maine.

\section{Dermochelys schlegelii (GARMaN)}

Sphargis coriacea var. schlegelii GARMAN

Bull. U. S. Nat. Mus., No. 25, 1881, p. 303.

Dermochelys schlegelii STEJNEGER

Bull. U. S. Nat. Mus., No. 58, 1907, p. 485.

TyPE LOCAlity: Tropical Pacific and Indian Ocean.

RANGE: Tropical Pacific Ocean; coasts of Lower California and Southern California.

\section{TRIONYCHIDAE (FAMILY)}

Amyda ( $G E N U S)$

Oken, Lehrb. Zool., Vol. 2, 1816, p. 348

TYPE: euphratica

SOFT-SHELLED TURTLES

\section{Amyda emoryi (Agassiz)}

Aspidonectes emoryi Agassiz

Contr. Nat. Hist. U. S., Vol. 1, 1857, p. 407, Vol. 2, pl. 6, figs. 4, 5. TyPe LOCALITy: Rio Grande River, near Brownsville, Texas. RANGE: Rivers of Texas, north into southern Oklahoma and Arkansas.

\section{Amyda ferox (SchNeIDER)}

Testudo ferox SchNeIDER

Naturg. Schildkr., 1783, p. 330.

Amyda ferox $\mathrm{OKEN}$

Lehrb. Zool., Vol. 2, 1816, p. 348.

TYPE LOCALITY : Savanna River, Georgia.

RANGE: South Carolina to Florida and Louisiana.

\section{Amyda mutica (Le Sueur)}

Trionyx muticus LE SueUR

Mém. Mus. Hist. Nat. Paris, Vol. 15, Dec. 1827, p. 263, pl. 7.

Amyda mutica Agassiz

Contr. Nat. Hist. U. S., 1857, Vol. 1, p. 399.

TyPE LOCALITY: Wabash River at New Harmony, Ind.

RANGE: Middle and Northern tributaries of the Mississippi and

St. Lawrence Rivers. 


Amyda spinifera (LE SUEUR)

Trionyx spiniferus Le Suevr

Mém. Mus. Hist. Nat. Paris, Vol. 15, Dec. 1827, p. 258, pl. 6. Amyda spinifera Hunteiz

Trans. Ac. Sci. St. Louis, Vol. 20, 1911, p. 251.

TYPE LOCALITY: Wabash River at New Harmony, Indiana.

RANGE: Mississippi River and tributaries west to Colorado, north to Montana; St. Lawrence River and tributaries; east to Vermont, western New York and Pennsylvania. 
PRINTED AT

THE HARVARD UNIVERSITY PRESS

CAMBRIDGE, MASS., U.S.A. 








